\title{
The Use of Diazomethane to Enhance the Selectivity of Bisphenol A Imprinted Polymers
}

By

\author{
Noof Alenazi
}

A thesis submitted to the Faculty of Graduate and Postdoctoral Affairs in partial fulfillment of the requirements for the degree of Master of Science

in

Chemistry

Carleton University

Ottawa, Ontario

(C) 2014, Noof Alenazi 


\section{Abstract}

Bisphenol A (BPA) is an estrogen-mimicking chemical that can be selectively extracted from water using a molecularly imprinted polymer (MIP). However, the presence of nonspecific binding sites has thusfar limited the utility of BPA-MIPs in sensor applications. This project explored two approaches to reduce or eliminate these sites: Optimizing the molar ratio of functional monomer (methacrylic acid) to cross-linker (ethylene glycol dimethacrylate) to template, or partially esterifying the carboxylic acid residues by siteselective chemical modification via treatment with diazomethane. Results show esterification with diazomethane to be more efficient in blocking non-specific binding sites than optimization of the template to monomer to crosslinker molar ratio. The efficiency and selectivity of the diazomethane-treated molecularly imprinted polymer (TMIP) particles were then compared with those of a commercially available MIP, either uniformly dispersed in the sample solution or densely packed into a cartridge, for solid phase extraction. Water and milk samples were spiked with BPA as well as zwitterionic, negatively and positively charged pharmaceuticals and other compounds for binding tests. Unlike high performance liquid chromatography, capillary electrophoresis (CE) demonstrated the ability to analyze milk samples after simple dilution with a background electrolyte. BPA was easily separated from all milk constituents on the basis of different electrophoretic mobilities. Repeatedly, CE binding test results demonstrated that the TMIP afforded greatly improved selectivity over the commercial MIP. 


\section{Acknowledgements}

First and foremost, I give praise to the Almighty God, the Lord of the Universe, for providing me with the opportunity to follow my dream and for granting me the capability to proceed it successfully.

I would like to express my deepest gratitude to my supervisors, Dr. Edward Lai and Dr. Jeffrey Manthorpe, for their excellent guidance, patience, scientific advice and insightful dissections throughout my research project. I never would have made it without them.

Special thanks go to my committee members for taking the time to read my dissertation. I would like to express gratitude to my scholarship sponsor, the Saudi Ministry of Higher Education, for providing me with a full scholarship.

My sincerest thanks also go to all of my colleagues in the Manthorpe Research Group and the Lai Research Group (present and past). They all provided me with the support, help, advice and friendship that I needed. In addition, I would like to thank Samar Alsudir for her help, advice, and encouragement throughout my study.

Last but not least, I would like to thank my parents, my sisters, and my brothers for their unconditional support and their best wishes throughout my degree.

Noof A. Alenazi 
This thesis is dedicated to the loving memory of my brother, Muhammad A. Alenazi (1987-2009).

“We don't remember days; we remember moments”. Cesare Pavese 


\section{Table of Contents}

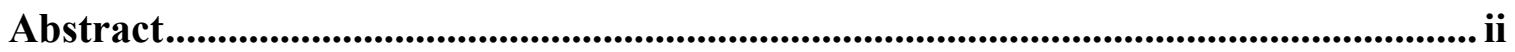

Acknowledgements ....................................................................................................ii

Table of Contents ................................................................................................................................ v

List of Tables ................................................................................................................................... viii

List of Figures........................................................................................................................... $\mathrm{x}$

List of Schemes ............................................................................................................. xii

List of Abbreviations ............................................................................................................... xiii

1 Chapter 1: Intoduction ........................................................................................................ 1

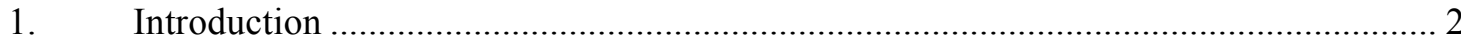

$1.1 \quad$ Molecularly Imprinted Polymer (MIP) …………………………………………….. 7

1.1.1 Functional Monomers................................................................................. 9

1.1.2 Cross-linking Molecules ............................................................................................ 10

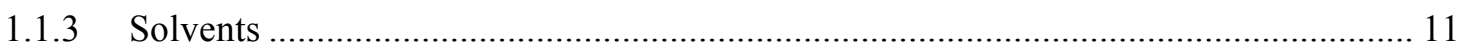

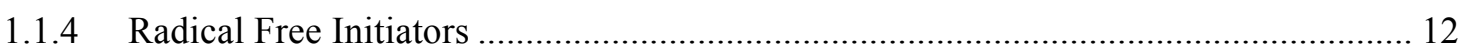

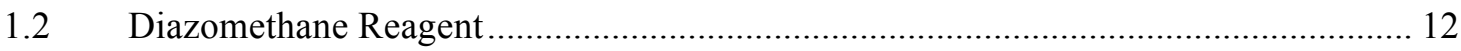

$1.3 \quad$ Chemical Post Modification of MIP............................................................................. 13

1.3.1 Unselective Technique ......................................................................................... 13

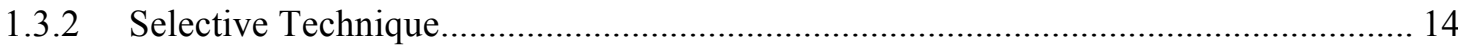

1.4 Capillary Electrophoresis Principles ……………………………………………... 15

1.4.1 Terminology of Electrophoresis ………………………………………………..... 16

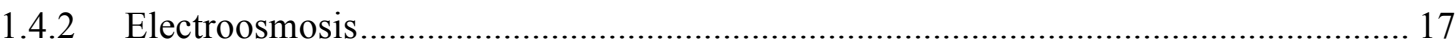

1.5 High-performance Liquid Chromatography Principle …………………………….... 18

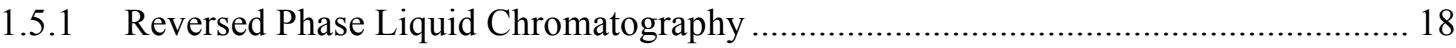

1.6 The separation efficiency of CE in comparison to HPLC ........................................... 19 


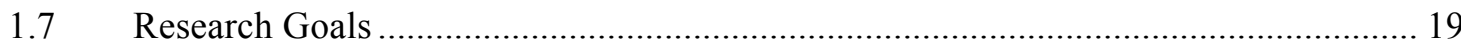

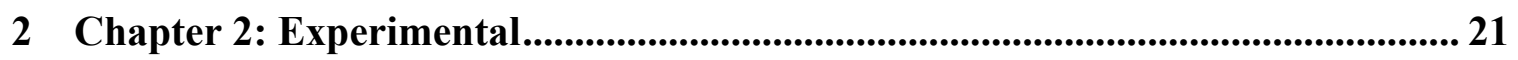

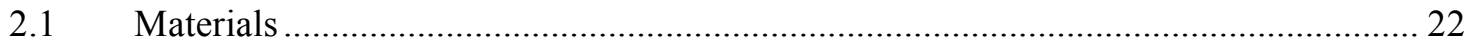

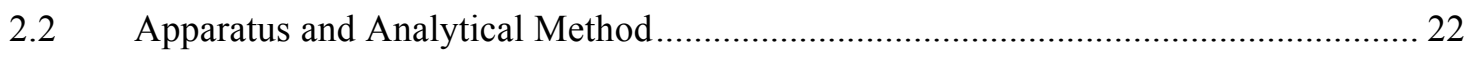

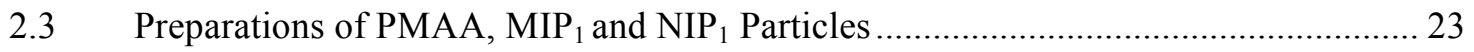

2.4 Optimized Molar Ratios of MAA to BPA in $\mathrm{MIP}_{2}$ to $\mathrm{MIP}_{10}$ and $\mathrm{NIP}_{2}$ to $\mathrm{NIP}_{10} \ldots \ldots \ldots . .23$

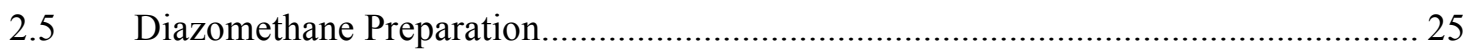

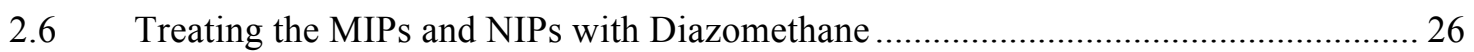

2.7 Competitive CE-UV Binding Tests of MIPs, TMIPS, NIPs, TNIP, and PMMA ........ 28

2.8 Competitive CE and HPLC Binding Tests of MIP ${ }_{6}$ and a Commercial MIP in Aqueous

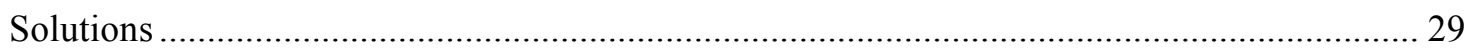

2.9 Competitive CE and HPLC Binding Tests of MIP ${ }_{6}$ and a Commercial MIP in Milk

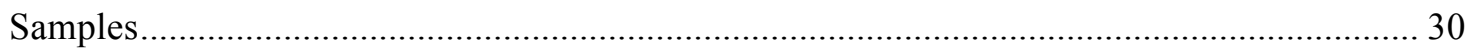

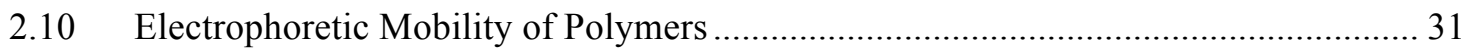

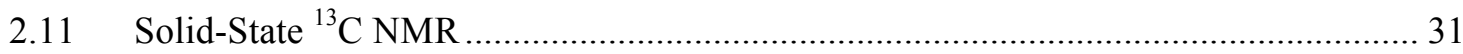

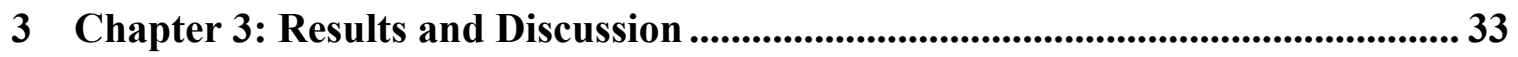

3.1 Modification of the Imprinted Polymer with Diazomethane ...................................... 33

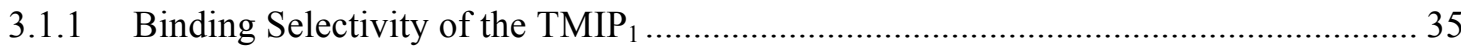

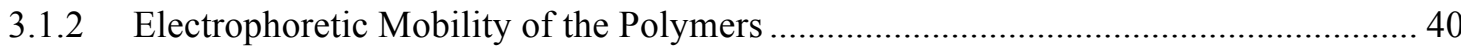

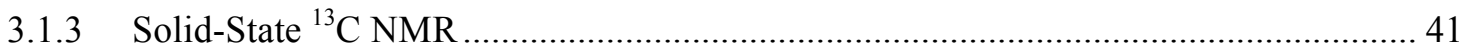

3.2 Optimization of Template to Functional Monomer Molar Ratio Approach ............... 45

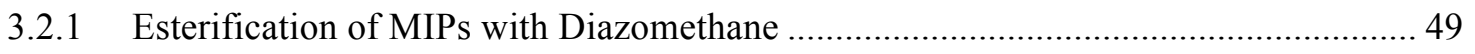

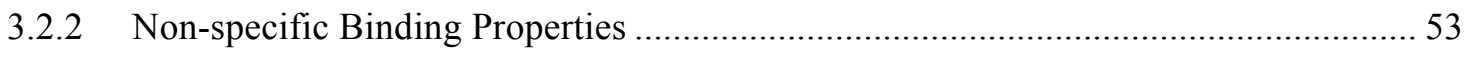

3.2.3 Average Size of MIPs and NIPs vs TMIPs and NTIPs ......................................... 57

3.3 Selective Extraction of BPA by TMIP $_{6}$ in Aqueous Solutions and Milk Samples ...... 59

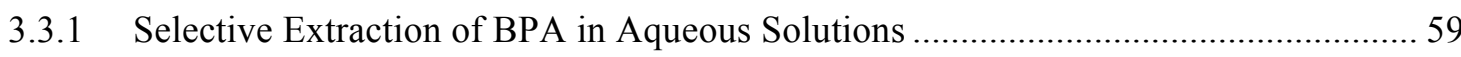


3.3.2 Selective Extraction of BPA in Milk Samples .

4 Chapter 4: Conclusions and Future Work ................................................................ 66

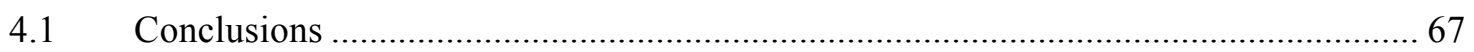

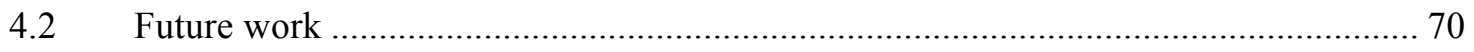

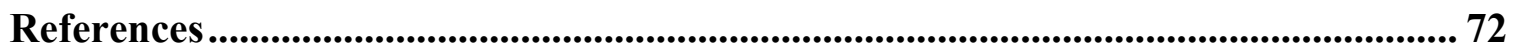




\section{List of Tables}

Table 2.1 Preparation of MIPs using different molar ratios of BPA to MAA while

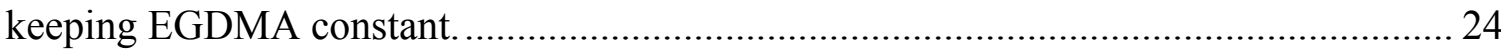

Table 3.1 Binding test results for a mixture of BPA and HMB with different polymers. The MIP ${ }_{1}$ and NIP $_{1}$, while suspended in 1:1 MeOH/THF, were treated with diazomethane to produce the TMIP* and TNIP*, respectively................................. 35

Table 3.2 Chemical structures and $\mathrm{pKa}$ values of BPA, DFC and MF................36

Table 3.3 Binding test results for BPA, DFC, MF and mixture (BPA+DFC+MF) with polymers 39

Table 3.4 Electrophoretic mobility values of $\mathrm{NIP}_{1}, \mathrm{MIP}_{1}, \mathrm{TMIP}_{1}, \mathrm{PMMA}$ and $\mathrm{TNIP}_{1} 41$

Table 3.5 Carbonyl signals in $\mathrm{NIP}_{1}, \mathrm{MIP}_{1}, \mathrm{TMIP}_{1}, \mathrm{TNIP}_{1}$, and PMMA

Table 3.6 Percent binding results for BPA, DFC and MF (individually or in mixture) and electrophoretic mobility for BPA MIPs 46

Table 3.7 Percent binding results for BPA, DFC and MF (individually or in mixture) and electrophoretic mobility for NIPs 48

Table 3.8 Percent binding results for BPA, DFC and MF (individually or in mixture) and electrophoretic mobility for TMIPs 51

Table 3.9 Percent binding results for BPA, DFC and MF (individually or in mixture) and electrophoretic mobility for various TNIPs 54

Table 3.10 Percent binding results for BFN (individually) with various MIPs, NIPs, TMIPs and TNIPs. 56

Table 3.11 HPLC-UV peak heights, peak areas and retention times for BFN, BPA, DFC and MF 55 
Table 3.12 Chemical structures and pKa values of BPA, BFN, CAF, DFC, DYE, FRU and MF ... 59

Table 3.13 Percent binding of commercial MIP cartridge, commercial MIP particles and TMIP6 particles with BPA, BFN, DFC and MF spiked in DDW, $10 \mathrm{mM} \mathrm{Na2HPO4}$ in DDW and DDW/MeOH/ CH3CN (1:1:1) based on CE-UV and HPLC-UV analyses 61 Table 3.14 Percent bindings of TMIP particles, commercial MIP particles and commercial MIP cartridge with BPA, BFN, CAF, DFC, DYE, FRU and MF spiked in milk samples, based on CE-UV analyses 64 


\section{List of Figures}

Figure 1.1 Illustration of non-covalent and covalent approaches in preparing

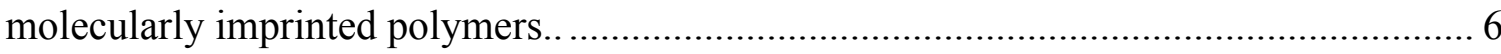

Figure 1.2 Chemical structure of methacrylic acid (MAA) .................................... 7

Figure 1.3 Chemical structure of EGDMA ........................................................ 8

Figure 1.4 Chemical structure of diazomethane …............................................. 10

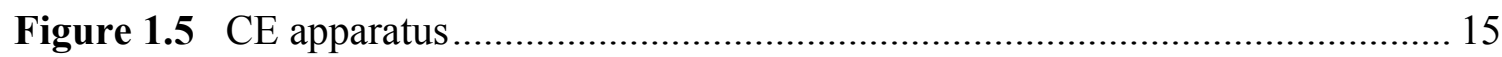

Figure 3.1 ${ }^{13} \mathrm{C}$ NMR spectra of $\mathrm{TMIP}_{1}$ (the pink line), $\mathrm{NIP}_{1}$ (the green line), $\mathrm{MIP}_{1}$

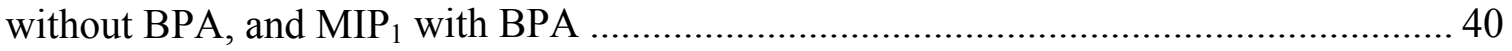

Figure 3.2 ${ }^{13} \mathrm{C}$ NMR spectrum of PMMA....................................................... 41

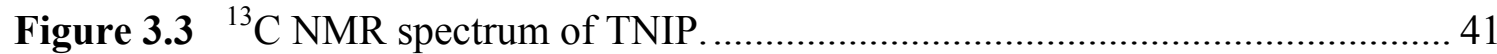

Figure 3.4 Percent binding results of BPA, DFC and MF (individually and in mixture) and electrophoretic mobility for each MIP prepared with a different mole $\%$ of BPA. For clarity, error bars are represented by the size of symbols......................................... 44

Figure 3.5 Percent binding results of BPA, DFC and MF (individually and in mixture) and electrophoretic mobility for each TMIP prepared with a different mole \% of BPA. For clarity, error bars are represented by the size of symbols.

Figure 3.6 Percent binding results of BFN (individually) for each MIP and TMIP prepared with a different mole \% BPA. For clarity, error bars are represented by the size

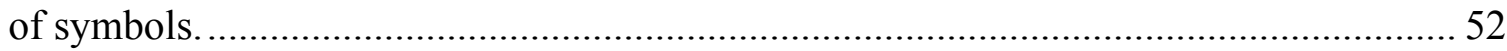


Figure 3.7 HPLC analysis of a mixture of BPA (100 ppm) at $2.5 \mathrm{~min}$, BFN (200 ppm) at $3.6 \mathrm{~min}, \mathrm{DFC}(200 \mathrm{ppm})$ at $4.1 \mathrm{~min}$ and $\mathrm{MF}(200 \mathrm{ppm})$ at $6.6 \mathrm{~min}$. Mobile phase: $\mathrm{CH} 3 \mathrm{CN} / \mathrm{MeOH} / \mathrm{DDW}(1: 1: 1 \mathrm{v} / \mathrm{v})$; flow rate, $0.8 \mathrm{~mL} / \mathrm{min}$; UV detection at $200 \mathrm{~nm} . \ldots . . .54$

Figure 3.8 SEM analysis of MIP, NIP, TMIP and TNIP particles. .......................... 56

Figure 3.9 DLS analysis of MIP, NIP, TMIP and TNIP particles.......................... 57

Figure 3.10 Electropherograms of milk spiked with DYE (a) before binding with commercial MIP particles, and (b) after binding with commercial MIP particles with no

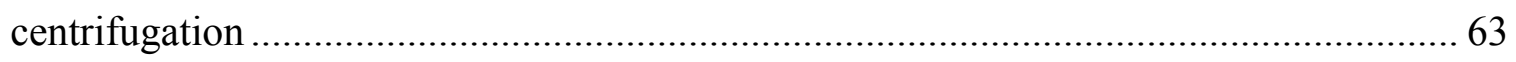

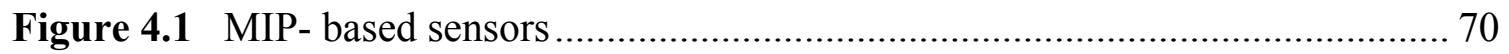




\section{List of Schemes}

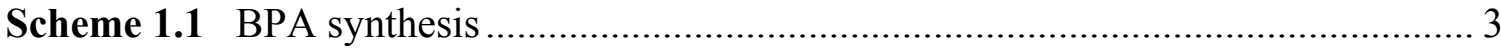

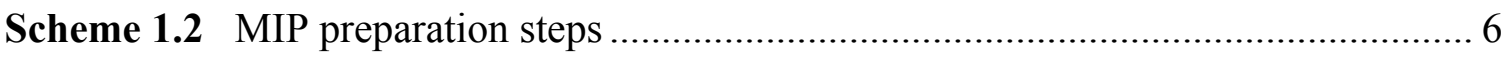

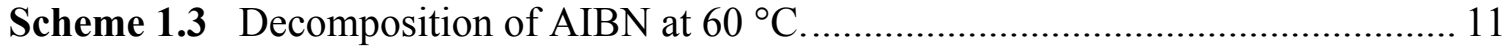

Scheme 2.1 Preparation of diazomethane .................................................................. 23

Scheme 2.2 Methylation of carboxylic acids with diazomethane .............................. 25 


\section{List of Abbreviations}

\begin{tabular}{|c|c|}
\hline $\mathrm{ACN}$ & acetonitrile \\
\hline AIBN & 2,2'-azobis(2-methylpropionitrile) \\
\hline $\mathrm{BFN}$ & baclofen \\
\hline BGE & background electrolyte \\
\hline BPA & bisphenol A \\
\hline $\mathrm{CAF}$ & caffeine \\
\hline $\mathrm{CE}$ & capillary electrophoresis \\
\hline DDW & deionized distilled water \\
\hline $\mathrm{DFC}$ & diclofenac sodium salt \\
\hline DYE & dyphylline \\
\hline EGDMA & ethylene glycol dimethacrylate \\
\hline FRU & fructose \\
\hline HMB & 2-hydroxy-4-methoxybenzophenone \\
\hline HPLC & high-performance liquid chromatography \\
\hline MAA & methacrylic acid \\
\hline $\mathrm{MeOH}$ & methanol \\
\hline MF & metformin/metformin hydrochloride \\
\hline MIP & molecularly imprinted polymer \\
\hline MMA & methyl methacrylate \\
\hline MO & mesityl oxide \\
\hline NIP & non-imprinted polymer \\
\hline NMR & nuclear magnetic resonance \\
\hline
\end{tabular}


PMMA poly(methyl methacrylate)

SPE solid phase extraction

SSCM site-selective chemical modification

TEA triethylamine

TMIP diazomethane-treated molecularly imprinted polymer

TNIP diazomethane-treated non-imprinted polymer

UV ultraviolet 
Chapter 1

\section{Introduction}




\section{Introduction}

Molecularly imprinted polymers (MIPs) provide analytical chemists with a family of highly selective sorbents for sample preparation [Martín-Esteban, 2013]. Many novel applications of MIPs in different formats have been reported for solid phase extraction [Turiel et al., 2010], stir bar sorptive extraction [Prieto et al., 2010] and magnetic beads dispersion extraction [Hu et al., 2013]. Targeting bisphenol A (BPA) in solid phase extraction (SPE) with MIPs is very important due the toxic effects of this compound in human health [Welshons et al., 2006, Vandenberg et al., 2007]. BPA has been classified by the US EPA as an endocrine disruptor compound (EDC), which is an exogenous agent that interferes with the action, binding, elimination, secretion, synthesis or transport of natural hormones in the body that are responsible for the maintenance of behaviour, development, homeostasis and reproduction [Vandenberg et al.,2009, Del Olmo et al. 1997]. BPA is also considered a potential factor in the increasing breast cancer rate among women in Korea [Yang et al., 2009], and it is correlated with obesity among children and adolescents [Trasande et al., 2012]. In addition, according to a study that measured BPA exposure of the US population, BPA was identified in $92.6 \%$ of individuals 6 years of age and older, with overall concentrations in urine ranging from 0.4 $\mu \mathrm{g} / \mathrm{L}$ to $149 \mu \mathrm{g} / \mathrm{L}$ [Calafat et al., 2008]. In Canada, the Federal Government has classified BPA as a "toxic substance" and added it to Schedule 1 of the Environmental Protection Act [Canada Gazette, 2013], thus permitting significant regulation of BPA through its full life cycle from "research and development, manufacturing, use, storage, transport, and ultimate disposal or recycling". 
BPA became popular in the production of many consumer products because it is inexpensive to manufacture and makes plastic products more durable. BPA is costeffective to manufacture because it is produced from acetone and phenol, which are cosynthesized in the cumene process (Hock process). This large-scale industrial production method relies on using two cheap materials (benzene and propylene) and converts them into more valuable materials (acetone and phenol) in the presence of $\mathrm{O}_{2}$ and a radical initiator [Ueda, 2010; Metrohm USA Ins., 2010] (Scheme 1.1).

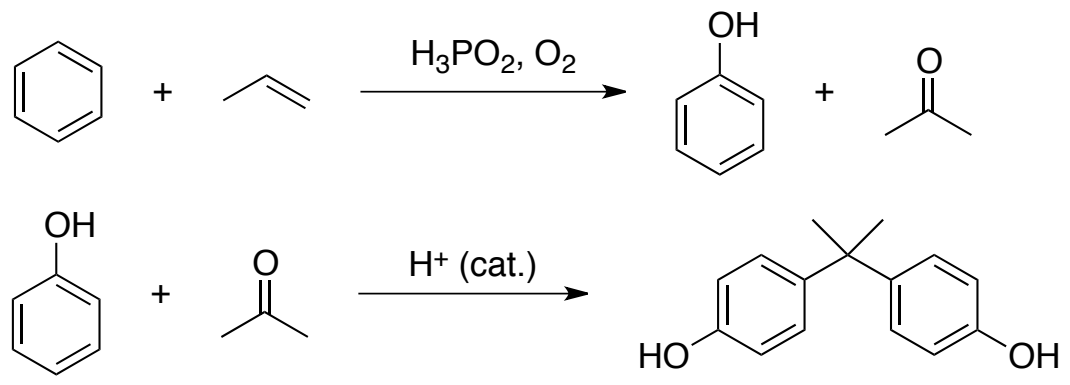

XS

Scheme 1.1 An industrial synthesis of BPA [Ueda, 2010].

BPA is of great concern due to its extensive use in the production of polycarbonate plastics, which are used as linings for most food and beverage cans, as additives in different consumer products [Umar et al., 2013], and as dental sealants [Calafat et al., 2008, Cho et al., 2012]. It is also used widely in the production of polyvinyl chloride plastics that are used for food packaging [Lau et al., 2000]. Annually, over three million metric tons of BPA are produced worldwide [Tsai, 2006]. BPA can be released into different environmental matrices during manufacturing or product disposal [Shao et al., 2007]. Due to its ability to dissolve in water, BPA has been detected in a 
number of rivers [Ren et al., 2014] in China [Xue et al., 2005], Germany [Quednow et al., 2008], Japan [Kashiwada et al., 2002; Kawahata et al., 2004] and Portugal [Rocha et al., 2013]. It is found in water along with other environmental contaminants such as pharmaceuticals and personal care products [Daughton, 2004, Stackelberg et al., 2007]. Analytical methods for BPA determination include high-performance liquid chromatography (HPLC), gas chromatography (GC), mass spectrometry (MS) and capillary electrophoresis [Sun at al., 2004, Mei et al., 2011]. For milk samples in particular, BPA has been determined by HPLC with UV detection [Ji et al., 2009], fluorescence detection [Prieto et al., 2010], electrochemical detection [Inoue et al., 2000] or tandem mass spectrometry [Gao et al., 2011].

Selective SPE techniques using MIPs are one practical approach to separate BPA from all other matrix components of the sample. In molecular imprinting, the target compound (such as BPA) is used as the template that interacts with a functional monomer (such as MAA) by non-covalent bonds. AIBN initiates the polymerization of MAA, with EGDMA serving as a cross-linker, to form a polymer matrix surrounding the BPA template molecules. Removal of the template molecules creates binding cavities with a size, shape and chemical functionality complementary to BPA, which is now the target compound in unknown samples. While MIPs provide a useful solid phase to extract a target analyte compound from water, there are several drawbacks including the leakage of any residual template, poor accessibility of the binding cavities in large particles, and existence of non-specific binding sites [Cormack et al., 2004]. Low-affinity binding sites in MIPs are considered serious problem, as these could generate false positive sensor signals due to non-specific binding [Krozer et al., 2010]. Minimizing the contact time 
with sample solutions readily prevents leakage of template molecules. Decreasing the size of, or reducing the degree of crosslinking in, MIP particles can alleviate poor accessibility of the specific binding cavities for the target molecules. Blocking the nonspecific binding sites in MIPs by esterification with diazomethane $\left(\mathrm{CH}_{2} \mathrm{~N}_{2}\right)$ could potentially improve the selectivity toward a target compound and diminish the occurrence of false positive signals. This concept was first suggested by Shimizu [Shimizu, 2005; Umpleby et al., 2001], who developed the site-selective chemical modification (SSCM) technique by esterification of non-specific carboxylic acid groups within binding sites with diazomethane or phenyldiazomethane in MIPs targeting ethyl adenine-9-acetate.

In this thesis, the SSCM approach was extended to an MIP targeting BPA. The selectivity of BPA binding with the modified and unmodified MIPs, as well as the corresponding non-imprinted polymer as a control, was examined. Treated MIP (TMIP) with diazomethane was tested for selective extraction of BPA in milk samples and compared with a commercially available MIP by using their particles of sub-micron size, either uniformly dispersed in the sample solution or densely packed into a cartridge, for SPE. Standard solutions were analyzed using capillary electrophoresis (CE) and highperformance liquid chromatography (HPLC) with ultraviolet (UV) detection, before and after the extraction of BPA in the presence of several pharmaceutical and other biologically relevant organic compounds. Baclofen (BFN) is a $\gamma$-aminobutyric acid agonist and is used mainly to relax muscles and to treat spasticity in children and adolescents [Duncan et al., 2013]. Caffeine (CAF) is a xanthine alkaloid and a stimulant drug that has recently caused the sudden death of a high school wrestler after taking a toxic dose [The Vancouver Sun, 2014.]. Diclofenac (DFC) sodium is a non-steroidal anti- 
inflammatory agent that is used in the treatment of inflammatory rheumatic and nonrheumatic conditions [Stierlin et al., 1979; Todd et al., 1988]. Dyphylline is a theophylline derivative for the treatment of asthma as well as chronic obstructive pulmonary diseases [Huang et al., 2003, Kester et al., 1987]. Fructose (FRU) is a monosaccharide, found in more than ten percent of the modern diet as corn syrup that is associated with diabetes, heart disease and weight gain [Dolson, 2006]. Metformin (MF) hydrochloride is a common drug for diabetes mellitus treatment, and there is preliminary evidence that it reduces the progression of several types of cancer tumors [Bao et al., 2014; Anisimov, 2014].

\subsection{Molecularly Imprinted Polymers (MIP)}

Molecular imprinted polymers (MIP) can be synthesized from template molecules, functional monomers, and cross-linking molecules. An initiator starts the polymerization of the functional monomers in organic solvents and in the presence of both the template molecules and a large excess of the cross-linking molecules, to form a polymer matrix surrounding the template molecules. The template can be an amino acid, protein,

nucleotide compound, pollutant, drug, or food compound [Vasapollo et al., 2011]. After polymerization, the template is removed from the polymer to create binding cavities with a size, shape and chemical functionality complementary to the template molecules [Cheong et al., 2013], as illustrated in Scheme 1.2. This synthetic polymer is then able to recognize its target compound through hydrogen bonding, dipole-dipole forces, and ionic interactions. 


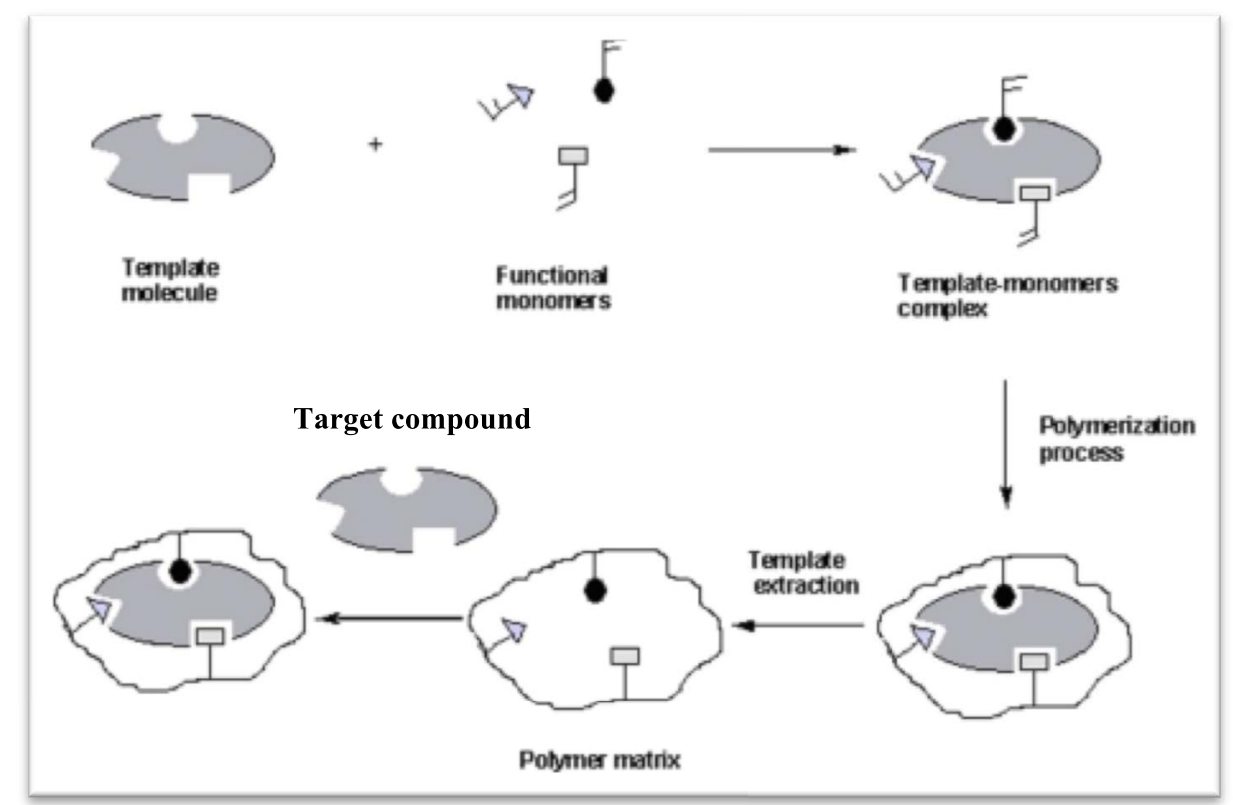

Scheme 1.2 MIP preparation steps [Cheong et al., 2013].

In the MIP technique, the interactions between functional monomers and template molecules are based on two approaches: the non-covalent approach developed by Mosbach and the covalent approach discovered by Wulff, as shown in Figure 1.1 [Haupt et al., 2000]. 


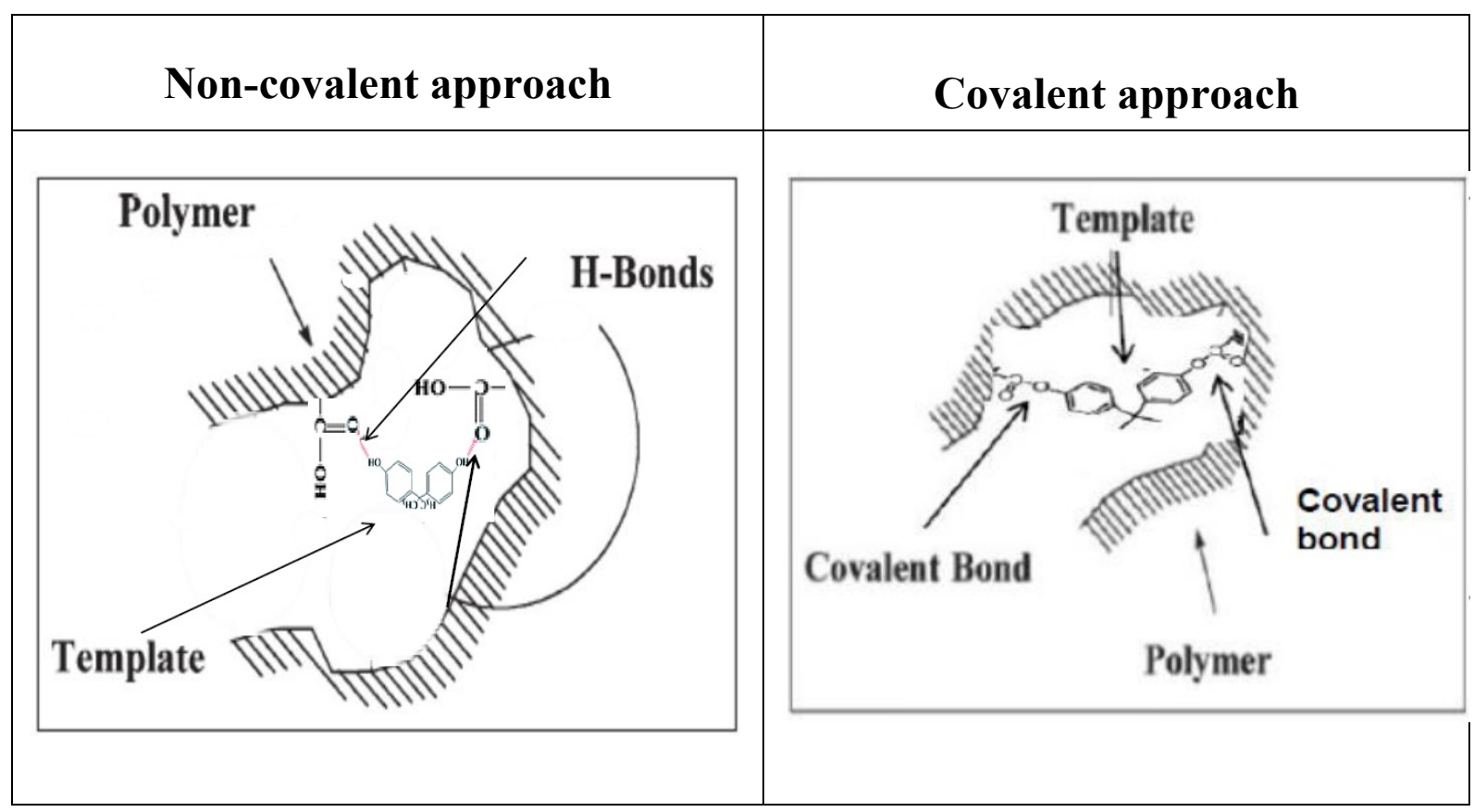

Figure 1.1 Illustration of non-covalent and covalent approaches in preparing molecularly imprinted polymers.

The covalent approach depends on the formation of covalent bonds between template molecules and functional monomers before the polymerization. Then, the template molecules are cleaved from the matrix by breaking these covalent bonds. The advantage of using the covalent approach is that it minimizes the number of non-specific binding sites in the MIP. This is due to the high stability of the template-monomer complex, which in turn provides a large quantity of specific binding sites [MartínEsteban, 2013]. However, the use of this covalent approach in MIP preparation is restricted because it can be only used for a limited number of template molecules (alcohols, ketones, amines, and carboxylic acids). Another drawback of this approach is that the rebinding process between the template and the monomer is slow because these covalent bonds need to be formed [Öpik et al., 2009]. On the other hand, the noncovalent method, which is based on hydrogen bonds and van der Waals, hydrophobic, 
and ionic interactions, is the most widely used approach for MIP preparation. This approach is common because of its simplicity, and it can be used for different types of monomers and template molecules [Tamayo et al., 2007]. However, the non-covalent approach depends on an equilibrium process of interaction between functional monomers and template molecules; therefore, in order to increase the stability of the templatemonomer complex, a large amount of monomer is used and this consequently leads to the presence of non-specific binding sites [Martín-Esteban, 2013].

Generally, MIPs display high selectivity toward their target compounds and exhibit resistance to high temperatures, strong acids, strong bases, and organic solvents. Furthermore, MIPs are not expensive to synthesize, and they have a long shelf life [Vasapollo et al., 2011].

\subsubsection{Functional Monomers}

The selection of functional monomers relies on several factors. One of them is that the functional monomers have to interact with the template molecules and form strong hydrogen bonds [Karim et al., 2005]. To increase the strength of these hydrogen bonds, functional monomers should be used in excess, which in turn could provide undesirable binding sites [Shimizu, 2005]. The price of monomers is also important and critical later on when MIP is used on a large scale as a SPE sorbent [Karim et al., 2005]. Functional monomers that can be used include basic monomers, such as vinylpyridine, acidic monomers, such as methacrylic acid, charged monomers, such as $N, N, N-$ trimethylaminoethyl methacrylate, hydrogen bonding monomers, such as acrylamide, and hydrophobic monomers, such as styrene. Among the acidic monomers, methacrylic acid 
(MAA) is the most widely used (Figure 1.2) [Haupt, 2001] because MAA is a good hydrogen donor and acceptor, so its non-covalent interactions with template molecules are readily reversed. Consequently, after removal of the template by an appropriate solvent, MAA allows rapid rebinding of the template [Vallano et al., 2000].

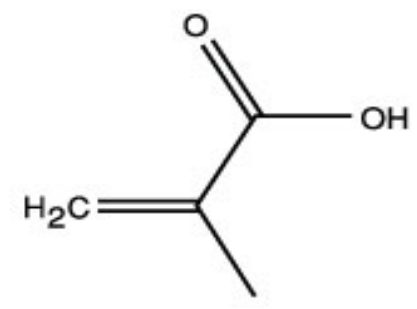

Figure 1.2 Chemical structure of methacrylic acid (MAA).

\subsubsection{Cross-Linking Monomers}

Cross-linking monomers have crucial functions in MIP preparation because they link the linear monomers together to establish a network. Cross-linking monomers control MIP morphology, fix recognition sites, and stabilize the functional monomers and template [Pardeshi et al., 2014]. Cross-linking molecules are usually used in high ratios to about $70-90 \%$ of the total MIP monomers as the polymer matrix needs a rigidity to retain the specific cavities' shape and preserve the imprinted recognition characteristics [Masque et al., 2001]. The cross-linking monomers also participate in the formation of specific binding cavities by interacting with functional monomers [Mullett et al., 1999]. By immobilizing the functional monomers in the polymer matrix, they actually help to rebind the target molecules with highly selective recognition [Vasapollo et al., 2011]. Ethylene glycol dimethacrylate (EGDMA) and trimethylolpropane trimethacrylate (TRIM) are the most commonly used cross-linking molecules in MIP preparation [Chen et al.,2011]; chemical structure of EGDMA and TRIM as shown in Figure 1.3. 
<smiles>C=C(C)C(=O)OCCOC(=O)C(=C)C</smiles>

Figure 1.3 Chemical structures of EGDMA (left) and TRIM (right).

\subsubsection{Porogens}

Solvents with pore-forming properties are called porogens [Masque et al., 2001]. In MIP preparation, the porogens are responsible for dissolving the template, functional monomers, and cross-linking molecules in the polymerization stage of MIP prepration, and they are responsible for production of macro-pores [Vasapollo et al., 2011]. The role of porogens is important in the formation of a stable complex between the template and functional monomers during MIP synthesis. Porogens should not interfere with hydrogen bonds formed between the template and functional monomers, so aprotic solvents with low polarities such as acetone, acetonitrile, tetrahydrofuran (THF), and diethyl ether are generally preferred. Porogens should also have small dielectric constants to increase the stability of the formation of the template-monomer complex because the more stable the complex, and hence the selectivity of the MIP. The H-bond parameter of porogens is another important factor in the stability of the template-monomer complex. Porogens such as acetone, acetonitrile, THF, and diethyl ether (with H-bond parameters of 6.1, 7, 8 [Pardeshi et al., 2014], and [7.05 Koenhen et al., 1975] respectively) are generally chosen. However, porogens such as methanol (with an H-bond parameter of 22.3) should be avoided because they may interfere with hydrogen bonds formed between the template 
and the functional monomers and consequently decrease the stability of the templatemonomer complex [Pardeshi et al., 2014].

\subsubsection{Radical Free Initiators}

Free radical reactions are beneficial in MIP preparation due to their electrical neutrality. These reactions are also useful because they remain uninfluenced by the presence of basic or acidic environments or by changes in the polarity of solvents. Free radical initiation can occur thermally at an elevated temperature or photochemically under UV irradiation. The most common initiator is 2, 2'-azobisisobutyronitrile (AIBN), which is decomposed thermally at $60{ }^{\circ} \mathrm{C}$ [Sellergren et al., 2000] as shown in Scheme 1.3 .

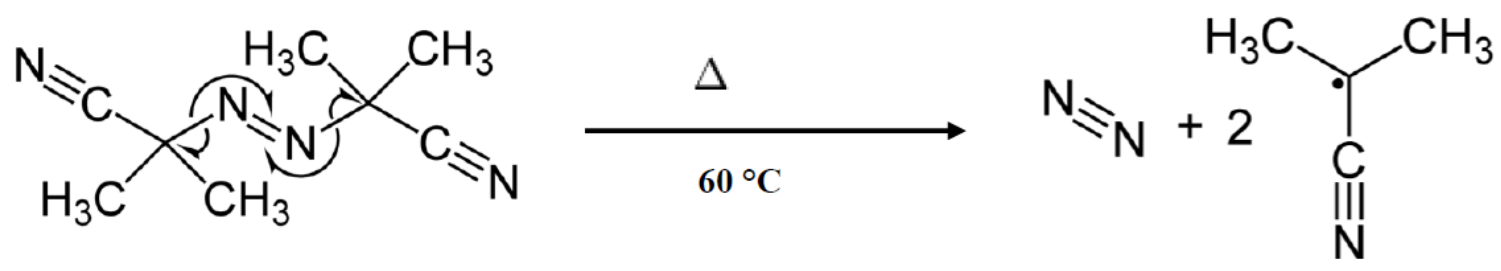

Scheme 1.3 Decomposition of AIBN at $60^{\circ} \mathrm{C}$.

\subsection{Diazomethane}

Diazomethane $\left(\mathrm{CH}_{2} \mathrm{~N}_{2}\right.$, Figure 1.4) was first discovered by Hans von Pechmann in 1895 [Roper et al., 1957]. It is used most commonly as a methylating reagent for carboxylic acids; it is also used to methylate phenols, enols, and certain nitrogen and sulfur functional groups. Diazomethane is usually prepared as a solution in ether because it forms a gas at room temperature; it becomes a liquid at $-23{ }^{\circ} \mathrm{C}$ and solidifies at $-145^{\circ} \mathrm{C}$. Although diazomethane, when handled safely, can be a harmless reagent, its distillation 
and use involve several safety precautions. Inhaled $\mathrm{CH}_{2} \mathrm{~N}_{2}$ vapor can induce chest pain, fever, and asthmatic symptoms. Diazomethane in crystal form reportedly can explode in an oversaturated solution [Sigma-Aldrich Technical Bulletin AL-180, 2007], and there have been reports of mortality from diazomethane [Roper et al., 1957]. Hence, it is crucial to avoid rough surfaces during diazomethane distillation, storage, and use. Clear, fire-polished joint glassware should be mandatory for its preparation because of this sensitivity [Sigma-Aldrich Technical Bulletin AL-180, 2007].

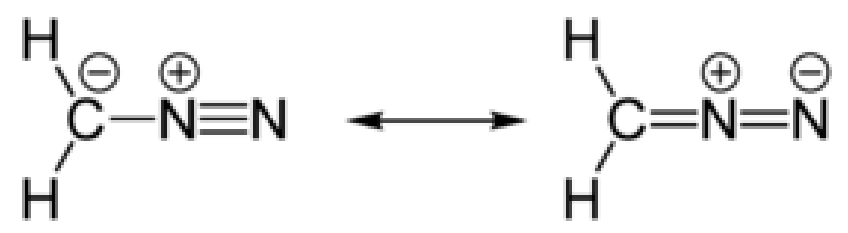

Figure 1.4 Chemical structure of diazomethane

\subsection{Chemical Modification of MIP}

Chemical modification of MIPs has been reported as a method to improve the binding properties in general for those chemically modified MIPs and also to enhance their selectivity and affinity toward their target analytes. The chemical modification of MIPs has been studied selectively and unselectively [Shimizu, 2005].

\subsubsection{Unselective Technique}

Sellergren and Shea reported unselective chemical post-modification of the functional monomers of an MAA/EGDMA matrix [Sellergren et al., 1993]. They used exhaustive esterification of MAA with diazomethane to test the hypothesis that the carboxylic acid protons are the key elements in the interactions with the template molecules through 
hydrogen bonding and electrostatic interactions. The result of their study showed a significant decrease in the selectivity of the esterified MIP in comparison with unesterified MIP, as well as a decrease in the affinity for all compounds [Sellergren et al., 1993; Shimizu, 2005]. Although this unselective approach did not work, it led to further investigation and subsequent development of the selective approach. Umpleby et al. [2001] then studied the unselective chemical post-modification of an MAA/EGDMA matrix imprinted with ethyl adenine-9-acetate (EA-9-A) with diazomethane or phenyldiazomethane. The results of this work agreed with Sellergren and Shea's work, showing a loss in the selectivity of the esterified MIP due to its unselective esterification because the template was removed prior to esterification [Umpleby et al., 2001; Shimizu, 2005].

\subsubsection{Selective Technique}

Selective chemical modification can improve the selectivity of MIPs. This technique employs both a selective reagent and guest-directed selectivity.

One method for selective chemical modification is to employ a size-selective reagent. An unmodified MIP contains small, medium, and large binding sites, so using a large reagent helps to access the large binding sites while leaving small and medium sites untreated. As a result, the modified MIP should be more selective toward smaller template molecules [Shimizu, 2005]. Frechet and Svec have used this technique successfully [Svec et al., 1996].

Another method for selective chemical modification is to protect the specific binding sites in the MIP and to modify only the unspecific binding sites. One can achieve this by using the template molecules to protect the specific binding sites during the chemical 
modification. McNiven et al. [1997] used this method to esterify MAA/EGDMA imprinted with testosterone; they esterified the polymer matrix with methyl iodide $\left(\mathrm{CH}_{3} \mathrm{I}\right)$ and 1,8-diazabicyclo[5.4.0]undec-7-ene, both in the presence and absence of the template molecules. They then compared the selectivity of the two modified MIPs by HPLC. The MIP modified prior to removal of the template molecules showed an improvement in the separation factor for testosterone (template/target) vs. progesterone, while the MIP modified in the absence of the template showed no difference in the separation factor compared to the original MIP [McNiven et al., 1997; Shimizu, 2005]. Umpleby et al. [2001] have also used a guest-directed selectivity method to improve the binding of EA9-A (target) through the esterification of MAA/EGDMA with diazomethane or phenyldiazomethane in the presence and absence of the template. Their results showed that the MIP esterified in the presence of the template yielded a higher percentage of specific binding sites in comparison with the MIP esterified in the absence of the template, which clearly provides evidence that the template molecule can protect the binding site during chemical modification with diazomethane [Umpleby et al. [2001, Shimizu, 2005].

\subsection{Capillary Electrophoresis Principles}

Capillary electrophoresis $(\mathrm{CE})$ is an analytical technique used to separate a mixture of compounds using an electrical field in a capillary tube [Glynn et al., 1998]. As shown in Figure 1.5, CE equipment consists of a capillary tube filled with buffer solution and positioned between two buffer reservoirs, with a high voltage field applied across it. A sample vial is introduced to the capillary inlet by replacing one buffer reservoir while a detector with an optical viewing window is placed at the outlet side of the capillary. The sample data are then acquired and saved by computer software [Bosserhoff et al., 2005]. 


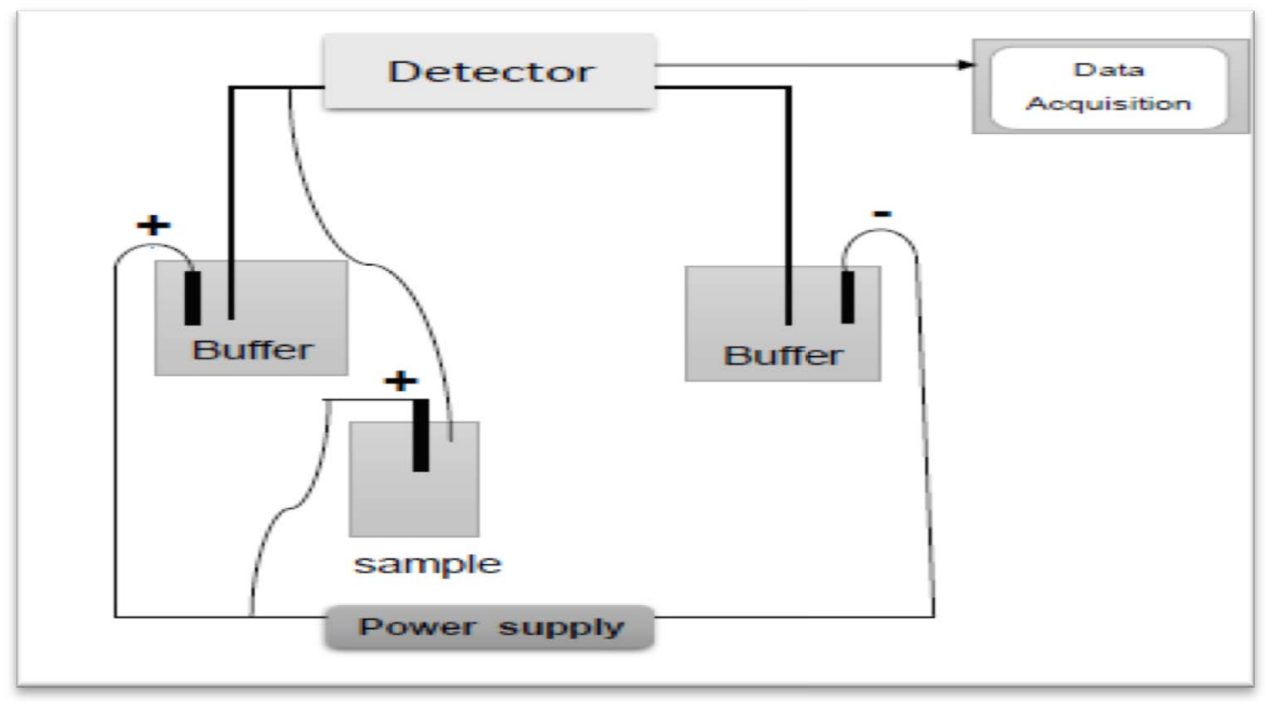

Figure 1.5 CE apparatus.

\subsubsection{Terminology of Electrophoresis}

Electrophoretic mobility, $\mu_{\mathrm{ep}}\left(\mathrm{cm}^{2} / \mathrm{V} \cdot \mathrm{s}\right)$, the electrophoretic velocity, $\mathrm{V}_{\mathrm{ep}}(\mathrm{cm} / \mathrm{s})$, and the strength of the electric field, E (V/cm), are important terms in CE. Equation 1 explains their relationships.

$$
\mu_{\mathrm{ep}}=\mathrm{V}_{\mathrm{ep}} / \mathrm{E}=\mathrm{L}_{\mathrm{d}} / \mathrm{t}_{\mathrm{m}} / \mathrm{V} / \mathrm{L}_{\mathrm{t}}
$$

From this equation, velocities are calculated by dividing the migration time $\left(\mathrm{t}_{\mathrm{m}}\right)$ by the capillary-to-detector length $\left(\mathrm{L}_{\mathrm{d}}\right)$ while motilities, which depend on voltage, capillary length, the buffer type, and the buffer $\mathrm{pH}$, are calculated by dividing the velocity $\left(\mathrm{V}_{\mathrm{ep}}\right)$ by the strength of the electrical field (E). In addition, the strength of the electrical field is calculated by dividing the voltage by the length of the entire capillary, $\mathrm{L}_{\mathrm{t}}$. Equation 1 determines the apparent mobility only; to determine the actual mobility, electroosmotic flow (EOF) must be included [Coulter, 1991]. 


\subsubsection{Electroosmosis}

EOF exists in any electrophoretic mechanism, and it is a critical factor in CE. EOF occurs whenever the liquid near a charged surface is placed in an electrical field, which results in the movement of any fluid near the surface inside the capillary. The electroosmotic flow velocity is known by the Smoluchowski equation:

$$
\mathrm{v}_{\text {eof }}=-(\varepsilon \zeta / 4 \eta \pi) \mathrm{E}
$$

Here $\varepsilon$ is the electrolyte (buffer) dielectric constant, $\zeta$ is the zeta potential (volts), $\eta$ is the viscosity (poise) and $\mathrm{E}$ is the applied voltage (volts/cm). The zeta potential measures the charge on the capillary wall, which depends on the material making up the capillary and the electrolyte (buffer) composition. Fused silica is the most common material for the capillary, with its surface hydrolyzed to give make it negatively charged, which attracts cations from the electrolyte (buffer) solution; this phenomenon is known as an electrical double layer. When an electrical field is applied, the cations migrate toward the cathode, moving water along and generating a pumping action. The zeta potential increases with the charge density on the surface. Note that the EOF velocity decreases with increasing concentration of the electrolyte (buffer). In summary, electroosmotic flow always moves toward the electrode that has the same charge as the capillary wall [Whatley, 2001].

\subsection{High-performance Liquid Chromatography Principles}

High-performance liquid chromatography (HPLC) is an analytical technique used to separate mixtures of compounds, including pharmaceutical, organic and ionic materials [Dong, 2006]. HPLC consists of a column packed with the stationary phase particles, a pump to move the mobile phase, and a detector to determine the retention times and 
concentrations of separated analytes. Retention time of an analyte is the time at which the analyte comes out at the exit end of the column. Retention times depend on the interaction between the stationary phase, the analyte molecules, and the solvents. When a sample is injected to the mobile phase stream, its components are hindered by the stationary phase interfaces. This hindrance relies on the analyte, stationary phase and mobile phase [Bansal, 2010].

\subsubsection{Reversed Phase Liquid Chromatography}

Reversed phase liquid chromatography (RP-HPLC) is a separation mode that was started in the mid-1970s [Tosoh Bioscience LLC, 2009]. In this mode, the stationary phase is non-polar while the mobile phase is polar or semi-polar. The RP-HPLC principle is based on hydrophobic interactions between the analyte, the stationary phase $\left(\mathrm{C}_{18}\right.$ column), and the mobile phase solvents [Bansal, 2010]. Introducing additional organic solvents into the mobile phase decreases the hydrophobic interactions between the analyte and the stationary phase, which results in desorption. Consequently, the more hydrophobic the analyte is, the longer the retention time. The RP-HPLC mode is the most common separation technique used in analytical chemistry because it is effective for almost all types of molecules, including charged and polar molecules [Tosoh Bioscience LLC, 2009].

\subsection{The Separation Efficiency of CE in Comparison to HPLC}

CE provides a unique and unparalleled resolution and separation in comparison to HPLC. The separation efficiency of CE and HPLC is governed by the Van Deemter equation 
(Equation 3), which relies mainly on the relationship between the plate height $(\mathrm{H})$ and the velocity $\left(v_{\mathrm{x}}\right)$ of the liquid along the separation axis $(\mathrm{x})$.

$$
\mathrm{H}=\mathrm{A}+\mathrm{B} / \mathrm{v}_{\mathrm{x}}+\mathrm{Cv}_{\mathrm{x}}
$$

Where $\mathrm{A}, \mathrm{B}$ and $\mathrm{C}$ are constants. When $\mathrm{H}$ is decreased, more theoretical plates $(\mathrm{N}$ $\sim 50,000$ to 500,000 ) can be generated along the separation axis. In CE, two terms in the Van Demeter equation are eliminated (zero): the multiple path term (A) and the masstransfer term $\left(\mathrm{Cv}_{\mathrm{x}}\right)$. Consequently, the only essential source of band broadening, under typical conditions, is from longitudinal diffusion $\left(\mathrm{B} / \mathrm{v}_{\mathrm{x}}\right)$. A typical separation by $\mathrm{CE}$ affords 50,000 to 500,000 theoretical plates, which is an order of magnitude better separation than HPLC (Xu, 1996).

\subsection{Research Goals}

The SSCM technique has been previously used to enhance the selectivity of MIPs toward their target analytes. This technique is used in the present project to enhance the selectivity of MIPs toward BPA as the target compound. This approach, by esterifying non-specific binding sites (namely MAA residues) in the MIP with diazomethane using diethyl ether as solvent and guest directed selectivity, has not been previously reported. In addition to the SSCM technique, another approach was attempted to enhance the selectivity of the MIP via optimization of the template to functional monomer molar ratio. The ultimate aim of those two approaches was to eliminate non-specific binding sites while keeping the specific biding sites intact in an MIP that was prepared using BPA as the template, MAA as the functional monomer, EGDMA as the cross-linker, and AIBN as the initiator. The third goal of this project was to examine the selectivity of our 
TMIP toward its target analyte, BPA, not only in aqueous solutions (BGE and DDW) but also in milk samples. These results were then compared with those of a commercially available MIP, either uniformly dispersed in the sample solution or densely packed into a cartridge, for solid phase extraction (SPE). Standard solutions were analyzed by CE and HPLC with UV detection, before and after the TMIP extraction of BPA in the presence of several pharmaceutical organic compounds and other biologically relevant organic compounds. The objective of this comparison was to explore the efficiency and selectivity of TMIP toward its target compound, BPA, without the interferences of other molecules in comparison with the commercial MIP. Hopefully, we can use our selective TMIP either to remove BPA from drinking water (in large-scale applications), infant milk and other food matrices or to employ the TMIP particles in sensor applications as a BPA receptor. 


\section{Chapter 2}

\section{Experimental}




\section{Experimental}

\subsection{Material}

Bisphenol A (BPA), baclofen (BFN), caffeine (CAF), diclofenac (DFC) sodium salt, dyphylline (DYE), 2-hydroxy-4-methoxybenzophenone (HMB), fructose (FRU) and metformin (MF) hydrochloride were all obtained from Sigma-Aldrich (Oakville, Ontario, Canada). 2,2'-azobis (2-isobutyronitrile) (AIBN) was acquired from Pfaltz \& Bauer (Waterbury, CT, USA). Diethyl ether was purchased from Fluka (Buchs, Switzerland). Sodium phosphate dibasic was supplied by Fisher Scientific (Fair Lawn, NJ, USA). All chemicals were used as received without any further purification except diethyl ether, which was distilled from lithium aluminum hydride. HPLC-grade methanol and acetonitrile $(\mathrm{ACN})$, and spectro-grade acetone, were all purchased from Caledon (Georgetown, ON, Canada). The AFFINIMIP ${ }^{\circledR}$ SPE BPA cartridges were purchased from AFFINISEP/ POLYINTELL (Paris, France). Sealtest 2\% partially skim milk (237 $\mathrm{mL}$ carton) was purchased at a local supermarket.

\subsection{Apparatus and Analytical Method}

A diazomethane generator kit ["Mini Diazald apparatus" (Aldrich part \# Z108898), Clear-Seal flask (Aldrich part \# Z100358); and a separatory funnel with PTFE stopcock and Clear-Seal joint (Aldrich part \# Z100382)] was obtained from Sigma-Aldrich (Oakville, ON, Canada). CE-UV analysis was performed on a laboratory-built system including a Spellman CZE1000R high voltage power supply (Hauppauge,NY, USA).

A fused-silica capillary $(51 \mu \mathrm{m}$ i.d., $356 \mu \mathrm{m}$ o.d.) was obtained from Polymicro Technologies (Phoenix, AZ, USA). The capillary for CE was $53.5 \mathrm{~cm}$ in total length and 
$46.1 \mathrm{~cm}$ in effective length to the detector. The BGE was consisted of $10 \mathrm{mM} \mathrm{Na}_{2} \mathrm{HPO}_{4}$ in deionized distilled water (DDW) to reach $\mathrm{pH} 7.5 \pm 0.2$. The capillary was flushed with pure methanol, 1.0 M HCl, 1.0 M NaOH, DDW, and the buffer (BGE). Prior to each use, the capillary was equilibrated with the run buffer at an applied voltage of $17 \mathrm{kV}$ for 10 min. A Bischoff Lambda1010 (Leonberg, Germany) UV detector was employed, at a wavelength of $190 \mathrm{~nm}$, to determine the compounds and find their migration time. This wavelength, $190 \mathrm{~nm}$, was chosen based on the BPA absorption in the UV and visible regions. The detector-acquired signal was obtained through a Peak Simple Chromatography Data System (SRI model 203, Torrance, CA, USA). The capillary was reconditioned by running the buffer at $17 \mathrm{kV}$ for $1 \mathrm{~min}$ in between sample analyses. The capillary inlet and outlet buffer solutions were changed after every ten CE analyses to keep the purity and level of BGE.

HPLC-UV analysis was carried out on a Shimadzu LC-GA system equipped with a 20 $\mu \mathrm{L}$ sample loop, a Phenomenex reversed phase C18 column $(4.6 \mathrm{~mm} \times 250 \mathrm{~mm}, 4 \mu \mathrm{m}$ packing material) and a Gilson 115 UV detector (Middleton, WI) set at $200 \mathrm{~nm}$. The absorbance signal was collected by a model N2000 chromatography workstation (Zhejiang University, Hangzhou, China). The mobile phase was acetonitrile (ACN), methanol $(\mathrm{MeOH})$ and DDW in 1:1:1 ratio at a flow rate of $0.8 \mathrm{~mL} / \mathrm{min}$.

\subsection{Preparations of PMAA, MIP 1 and NIP 1 Particles}

BPA-imprinted polymer (MIP1) and non-imprinted polymer (NIP1) particles were prepared following a procedure by Alsudir et al. [2012]. BPA (the template), MAA (the functional monomer), and EGDMA (the cross-linker) were combined in a molar ratio of 
1:8:7, and dissolved in a mixture of acetone/ACN $(1: 3 \mathrm{v} / \mathrm{v})$. The initiator, AIBN, was added to the mixture (at $2 \%$ by weight of the monomers). After sonication and deoxygenation by bubbling nitrogen gas through the mixture for $5 \mathrm{~min}$, the reaction vial was sealed and placed in a $60{ }^{\circ} \mathrm{C}$ water bath for $24 \mathrm{~h}$ (without further mixing or shaking) to produce submicron MIP1 particles, which were then rinsed six to seven times with 10 $\mathrm{ml}$ of $5 \%$ TEA in methanol for each rinse to wash out the template molecules. The particles were then rinsed three times with $10 \mathrm{ml}$ of $\mathrm{ACN}$ for each rinse to remove any remaining TEA. Centrifugation of polymer particles was carried out at $10000 \mathrm{rpm}$ for 10-20 min, and the supernatants were discarded. Finally, the MIP1 particles were dried overnight in an oven at $50{ }^{\circ} \mathrm{C}$. NIP particles were prepared analogously but in the absence of the template molecule (BPA). For a direct comparison of NMR peaks, poly(methyl methacrylate) (PMMA) was prepared in the same manner using methyl methacrylate (MMA) instead of MAA as the functional monomer [Alsudir et al., 2012].

\subsection{Optimized Molar Ratios of MAA to BPA in $\mathrm{MIP}_{2}$ to $\mathrm{MIP}_{10}$ and NIP $_{2}$ to NIP 10}

$\mathrm{MIP}_{2}$ to MIP 10 were prepared using different molar ratios of BPA to MAA while keeping EGDMA constant at $7 \mathrm{mmol}$, (Table 2.1). All MIPs in Table 2.1 were dissolved in a mixture of acetone/acetonitrile $(1: 3 \mathrm{v} / \mathrm{v})$. AIBN was added (at $2 \%$ by weight of the prepolymerization mixture except the solvents) as an initiator. After sonication and deoxygenation by bubbling nitrogen gas through the mixture for $5 \mathrm{~min}$, the reaction vial was sealed and placed in a $60{ }^{\circ} \mathrm{C}$ water bath for $24 \mathrm{~h}$ (without further mixing or shaking) to produce submicron MIP particles, which were then collected by centrifugation at 
$10000 \mathrm{rpm}$ for $10-20 \mathrm{~min}$ and rinsed seven times each with $10 \mathrm{ml}$ of $5 \%$ TEA in methanol to wash out the template molecules. The particles were then rinsed three times each with $10 \mathrm{ml}$ of acetonitrile to remove any remaining TEA. Finally, all the MIP particles were dried overnight in an oven at $50{ }^{\circ} \mathrm{C}$. For direct comparisons of binding tests results, NIPs were prepared analogously to MIPs but in the absence of BPA.

Table 2.1 Preparation of MIPs using different molar ratios of BPA to MAA while keeping EGDMA constant.

\begin{tabular}{|c|c|c|c|c|}
\hline & \multicolumn{3}{|c|}{ Molar ratio } & \multirow{2}{*}{ Molecularly imprinted polymer BPA } \\
\cline { 2 - 4 } & $\begin{array}{c}\text { BPA } \\
(\mathrm{mmol})\end{array}$ & $\begin{array}{c}\text { MAA } \\
(\mathrm{mmol})\end{array}$ & $\begin{array}{c}\text { EGDMA } \\
(\mathrm{mmol})\end{array}$ & \\
\hline $\mathrm{MIP}_{2}$ & 1 & 9 & 7 & 0.06 \\
\hline $\mathrm{MIP}_{3}$ & 2 & 8 & 7 & 0.12 \\
\hline $\mathrm{MIP}_{4}$ & 3 & 7 & 7 & 0.18 \\
\hline $\mathrm{MIP}_{5}$ & 4 & 6 & 7 & 0.24 \\
\hline $\mathrm{MIP}_{6}$ & 5 & 5 & 7 & 0.29 \\
\hline $\mathrm{MIP}_{7}$ & 6 & 4 & 7 & 0.35 \\
\hline $\mathrm{MIP}_{8}$ & 7 & 3 & 7 & 0.41 \\
\hline $\mathrm{MIP}_{9}$ & 8 & 2 & 7 & 0.47 \\
\hline $\mathrm{MIP}_{10}$ & 9 & 1 & 7 & 0.53 \\
\hline
\end{tabular}




\subsection{Diazomethane Preparation}

Due to the potentially explosive and toxic nature of diazomethane, this reaction was performed behind a safety shield in a fume hood! Diazomethane was prepared from $\mathrm{N}$ methyl- $N$-nitroso-para-toluenesulfonamide (Diazald $®$ ), Scheme 2.1, [De Boer et al., 1954] following a literature procedure [Sigma-Aldrich Technical Bulletin AL-180, 2007] as a solution in ether with trace amounts of ethanol using an Aldrich mini Diazald apparatus.

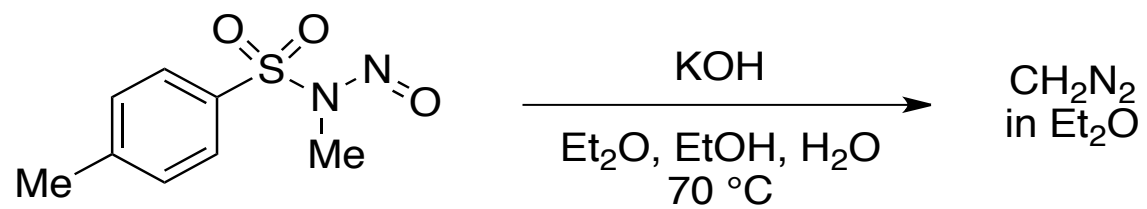

Scheme 2.1 Preparation of diazomethane

Specifically, $10 \mathrm{ml}$ of $95 \%$ ethanol and $8 \mathrm{ml}$ of water were added to $5 \mathrm{~g}$ of $\mathrm{KOH}$ in the reaction vessel portion of the apparatus. The resulting mixture was then stirred and heated in an oil bath to $65^{\circ} \mathrm{C}$. A separatory funnel containing $5 \mathrm{~g}$ of Diazald dissolved in $45 \mathrm{ml}$ of diethyl ether was placed over the reaction vessel. The cold finger was cooled with dry ice and isopropanol, and a $100 \mathrm{ml}$ receiving flask was attached. When the reaction vessel warmed to $65{ }^{\circ} \mathrm{C}$, the solution of Diazald in ether was added over a period of 20 minutes so that the rate of distillation matched the rate of the addition of the Diazald solution. The final solution of $\mathrm{CH}_{2} \mathrm{~N}_{2}$ gas dissolved in diethyl ether was protected from shock and stored in a freezer $\left(\sim-15^{\circ} \mathrm{C}\right)$. 


\subsection{Treating the MIPs and NIPs with Diazomethane}

Due to the potentially explosive and toxic nature of diazomethane, this reaction was performed behind a safety shield in a fume hood! The diazomethane-treated molecularly imprinted polymers (TMIPs) and diazomethane-treated non-imprinted polymers (TNIPs) were prepared analogously to the MIPs and NIPs as described in section 2.3 and 2.4, and dissolved in a mixture of acetone/ACN $(1: 3 \mathrm{v} / \mathrm{v})$. The initiator, AIBN, was added to the mixture (at $2 \%$ by weight of the monomers). After sonication and deoxygenation by bubbling nitrogen gas through the mixture for $5 \mathrm{~min}$, the reaction vial was sealed and placed in a $60{ }^{\circ} \mathrm{C}$ water bath for $24 \mathrm{~h}$ (without further mixing or shaking) to produce submicron MIPs particles. Centrifugation of polymer particles was carried out at 10000 rpm for 15 min and the supernatants were discarded. The MIP particles were then dried overnight in an oven at $50{ }^{\circ} \mathrm{C} .670 \mathrm{mg}$ of MIP particles (still containing the BPA template, as it had not been washed out) were suspended in $50 \mathrm{~mL}$ of a 1:1 mixture of THF and $\mathrm{MeOH}$ to produce TMIP $*$ and TNIP* or in diethyl ether to produce $\mathrm{TMIP}_{1}$ to TMIP $_{10}$ and TNIP ${ }_{10}$ TNIP ${ }_{10}$. Ethereal diazomethane solution was added to the MIPs and NIPs suspensions in order to methylate the carboxylic acid groups in the MAA/EGDMA matrix (Scheme 2.2). The reaction was deemed complete when the yellow color of diazomethane persisted for several minutes. Nitrogen gas was then used to purge the remaining diazomethane from the reaction mixture, and the mixture was concentrated on a rotary evaporator at $40{ }^{\circ} \mathrm{C}$ to afford treated molecularly imprinted polymers and treated non-imprinted polymers: TMIPs and TNIPs. In order to remove the template molecules from TMIPs particles were rinsed seven times with $10 \mathrm{ml}$ of 5\% TEA in methanol for each rinse; the particles were then rinsed three times with $10 \mathrm{ml}$ of ACN for each rinse to 
remove any residual TEA. The polymer particles were precipitated by centrifugation at $10000 \mathrm{rpm}$ for $15 \mathrm{~min}$, and the supernatants were discarded. The TMIPs and TNIPs particles were then dried overnight in an oven at $50{ }^{\circ} \mathrm{C}$.

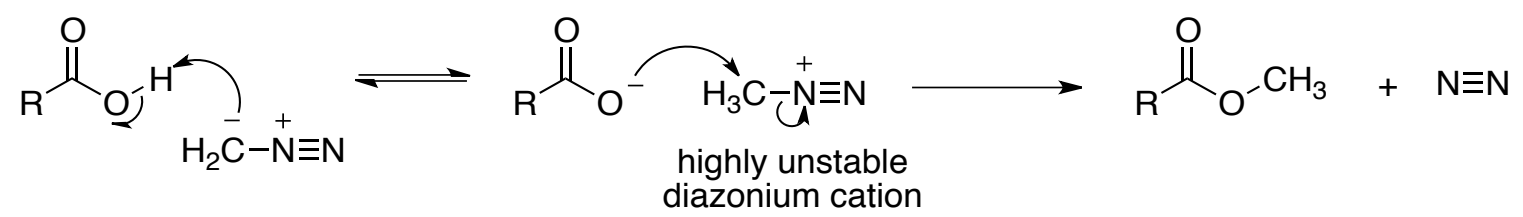

Scheme 2.2 Methylation of carboxylic acids with diazomethane

MIP* and NIP* particles were suspended in a 1:1 mixture of THF and $\mathrm{MeOH}$ during the reaction with diazomethane to generate TMIP $*$ and TNIP* or in a diethyl ether solvent to generate $\mathrm{TMIP}_{1}$ to $\mathrm{TMIP}_{10}$ and $\mathrm{TNIP}_{1}$ to $\mathrm{TNIP}_{10}$.

\subsection{Competitive CE-UV Binding Tests of MIPs, TMIPS, NIPs, TNIP, and PMMA}

Ten milligrams per milliliter of PMMA, MIPs, and TNIPs particles were prepared in 10 $\mathrm{mM} \mathrm{Na}_{2} \mathrm{HPO}_{4} \mathrm{BGE}(\mathrm{pH} 7.5 \pm 0.2)$. Standard stock solutions of $200 \mathrm{ppm}$ BPA, $5000 \mathrm{ppm}$ $\mathrm{HMB}, 800 \mathrm{ppm}$ of BFN, DFC and MF were prepared in the BGE. Working standard solutions of 100 ppm BPA and 200 ppm BFN, 200 ppm DFC, $200 \mathrm{HMB}$, and $200 \mathrm{MF}$ were prepared when needed. Batch-mode binding tests were used to evaluate the binding between the particles and target compounds. After sonication for 20 minutes, the solution was centrifuged for 10 minutes, and the concentrations of BPA, BFN, DFC and MF in the supernatant were analyzed by CE-UV analysis. An individual run of mesityl oxide (MO) as the neutral marker was carried out for ionic charge determination of the target compounds. Selectivity of the PMMA, MIP, NIP, TMIP and TNIP particles were tested 
using the mixture of BPA, DFC and MF or using BFN only in the run buffer, respectively while the selectivity of MIP*, NIP*, TMIP* and TNIP* were determined using BPA and HMB. Peak areas were measured for each compound before and after binding with PMMA, MIP, NIP, TMIP, and TNIP particles. All binding tests were carried out in duplicate, and percentage binding was calculated as [(peak area before binding-peak area after binding)/peak area before binding] $\times 100$.

\subsection{Competitive CE and HPLC Binding Tests of $\mathrm{MIP}_{6}$ and a}

\section{Commercial MIP in Aqueous Solutions}

Ten milligrams per milliliter of MIP $_{6}$ particles or commercial MIP particles were suspended in either $10 \mathrm{mM} \mathrm{Na}_{2} \mathrm{HPO}_{4} \mathrm{BGE}(\mathrm{pH} 7.5 \pm 0.2)$ or DDW. Standard stock solutions of $200 \mathrm{ppm}$ BPA, $800 \mathrm{ppm}$ BFN, $800 \mathrm{ppm}$ DFC, and 800 ppm MF were prepared in the same BGE or in DDW. Working standard solutions of 100 ppm BPA, 200 ppm BFN, 200 ppm DFC, and 200 ppm MF were prepared freshly prior to each binding test. Batch-mode binding tests were used to evaluate the binding between the particles and test compounds under sonication for 20 minutes. After the particles were precipitated by centrifugation at $10000 \mathrm{rpm}$ for 10 minutes, the concentrations of BPA, DFC and MF, or BFN, in the supernatant were determined by CE-UV and HPLC-UV analysis. An individual run of mesityl oxide (MO) as the neutral marker was carried out for ionic charge determination of the test compounds and particles by CE-UV analysis. In the case of MIP cartridges (containing $100 \mathrm{mg}$ of particles), $2 \mathrm{~mL}$ of BGE or DDW solution, spiked either with a mixture of 100 ppm BPA, 200 ppm DFC and 200 ppm MF, or individually with $200 \mathrm{ppm}$ BFN, was loaded for SPE. The eluate was then directly analyzed by CE-UV without sonication or centrifugation. Peak areas were measured for 
each compound before and after binding. All binding tests were carried out in duplicate, and percentage binding was calculated as [(peak area before binding - peak area after binding)/peak area before binding] $\times 100$.

\subsection{Competitive CE and HPLC Binding Tests of $\mathrm{MIP}_{6}$ and a Commercial MIP in Milk Samples}

All stock solutions of 200 ppm BPA, 800 ppm BFN, 175800 ppm CAF, 800 ppm DFC, $800 \mathrm{ppm}$ DYE, and $800 \mathrm{ppm}$ MF were prepared in the BGE. Half a milliliter of milk was spiked with the stock solutions and diluted with BGE to a final volume of $2 \mathrm{~mL}$, thus containing 100 ppm BPA, 200 ppm BFN, 200 ppm CAF, 200 ppm DFC, 200 ppm DYE, 30000 ppm FRU or 200 ppm MF. Ten milligrams per milliliter of either TMIP or commercial MIP particles were dispersed into the spiked milk samples via sonication for 20 minutes. After the particles were precipitated by centrifugation at $10000 \mathrm{rpm}$ for 10 min, the supernatant was analyzed by CE-UV to determine the final concentrations of BPA, BFN, CAF, DFC, DYE, FRU and MF. In the case of commercial MIP cartridges, two milliliters of milk sample was spiked either with a mixture of $100 \mathrm{ppm}$ BPA and 200 ppm MF, or individually with 200 ppm BFN, 200 ppm DYE or 30000 ppm FRU. The spiked milk sample was allowed to percolate, under atmospheric pressure, through the

densely packed cartridge. The eluate was directly analyzed by CE-UV without centrifugation. 


\subsection{Electrophoretic Mobility of Polymers}

The negative or positive charges of MIPs, NIPs, TMIPs and TNIPs particles were determined by CE-UV analysis after they were suspended in the BGE with ultrasonication. The electrophoretic mobility $M$ of each polymer was calculated by

$$
M=\left(\mathrm{L}_{\mathrm{tot}} \mathrm{L}_{\mathrm{d}} / \mathrm{V}\right)\left(1 / \mathrm{t}_{\mathrm{p}}-1 / \mathrm{t}_{\mathrm{ref}}\right)
$$

Where $L_{\mathrm{d}}$ is the capillary length from the inlet to the detector, and $V$ is the high voltage $(20 \mathrm{kV})$ applied across the entire capillary length $\left(\mathrm{L}_{\mathrm{tot}}\right) ; \mathrm{t}_{\mathrm{ref}}$ and $\mathrm{t}_{\mathrm{p}}$ are the migration times of the neutral (reference) marker and the particles, respectively.

\subsection{Solid-State ${ }^{13}$ C NMR}

All ${ }^{13} \mathrm{C}$ cross polarization/magic angle spinning NMR spectra were obtained on a Bruker Avance III 400 (400 MHz, 9.4 T) or Avance III 200 (200 MHz, 4.7 T) spectrometer at a spinning speed of $10 \mathrm{kHz}$. 


\section{Chapter 3}

\section{Results and}

Discussion 


\section{Results and Discussion}

\subsection{Modification of the Imprinted Polymer with Diazomethane}

Diazomethane reacts very rapidly with carboxylic acid groups to form the corresponding methyl esters. We sought to use the high reactivity of diazomethane as a technique to modify MIPs by suspending the imprinted polymer in the presence of the template (in this case, BPA), wherein the template acts as an in situ protecting group for the specific binding sites and allowing the non-specific carboxylic groups to selectively react with $\mathrm{CH}_{2} \mathrm{~N}_{2}$. Umpleby et al. [2001] proposed that the template has the ability to protect the high-affinity binding sites of the template in the MIP and keep them inactivated during the treatment with $\mathrm{CH}_{2} \mathrm{~N}_{2}$ Umpleby et al., 2001, Shimizu, 2005].

In related work, McNiven et al. [1997] demonstrated the importance of the presence of the template during the esterification of an MAA/EGDMA polymer imprinted with testosterone. When the MIPs were partially esterified with methyl iodide in the presence and absence of the template and by applying HPLC to compare the selectivity of these treated polymers, they noticed an improvement in the separation of testosterone (the template) vs. progesterone [McNiven et al., 1997].

Another important aspect of this work was the choice of solvent. Our selection of the moderately polar diethyl ether was precipitated by its modest hydrogen bonding ability (with the H-bond parameter of 7.05 [Pardeshi et al., 2014]) [Snyder, 1978], which would thus provide minimal disruption of the hydrogen bonds between the template and the high affinity binding sites. However, we also postulated that a certain amount of hydrogen bonding by the solvent during the reaction of the polymer with diazomethane 
may be beneficial in minimizing non-specific binding of BPA, thus resulting in an even greater selectivity in site-selective chemical modification (SSCM) of the MIP. According to Nicholls et al. [1995], the presence of the water considerably decreases the effectiveness of hydrogen bonding between ligand and receptor due to the increased competition for hydrogen bond donor and acceptor sites. Similarly, we observed that when the treatment was performed by addition of ethereal diazomethane to a suspension of the MIP in a 1:1 mixture methanol and THF, the binding cavities were virtually completely methylated - this TMIP (TMIP*) showed the same results as a control [treated non-imprinted polymer (TNIP*)] when BPA and 2-hydroxy-4-methoxybenzophenone (HMB) were chosen to carry out binding tests with TMIP $*$ and TNIP* as shown in Table 3.1. These results demonstrate the lack of selective binding sites in both TMIP* and TNIP*. Similarly, the NIP ${ }_{1}$ demonstrated modest and non-selective binding of BPA, while the MIP 1 , as expected, showed significantly higher BPA affinity. This is consistent with the findings of Shimizu et al. [Shimizu, 2005] that high affinity binding sites seem to be preferentially esterified during SSCM when the template is absent; thus, the observed binding is strictly due to binding to non-selective sites [Umpleby et al., 2001]. The higher binding affinity of HMB over BPA is a result of its five-fold higher concentration and its documented high affinity for non-selective binding sites [Alsudir et al., 2012 and 2013]. 
Table 3.1 Binding test results for a mixture of BPA and HMB with different polymers. The MIP 1 and $\mathrm{NIP}_{1}$, while suspended in 1:1 MeOH/THF, were treated with diazomethane to produce the $\mathrm{TMIP}_{*}$ and $\mathrm{TNIP} *$, respectively.

\begin{tabular}{|l|l|}
\hline Polymer & $\%$ Binding $^{\mathrm{a}}$ \\
\hline MIP $_{1}$ & $\mathrm{BPA}^{\mathrm{b}}=90 \% \pm 1 \%$ \\
& $\mathrm{HMB}^{\mathrm{c}}=99 \% \pm 1 \%$ \\
\hline TMIP $^{*}$ & $\mathrm{BPA}^{\mathrm{b}}=48 \% \pm 1 \%$ \\
\hline $\mathrm{NIP}_{1}$ & $\mathrm{HMB}^{\mathrm{c}}=99 \% \pm 1 \%$ \\
\hline TNIP $_{*}$ & $\mathrm{BPA}^{\mathrm{b}}=71 \% \pm 5 \%$ \\
& $\mathrm{HMB}^{\mathrm{c}}=80 \% \pm 1 \%$ \\
\hline
\end{tabular}

${ }^{\text {a}}$ Percentage binding was determined by CE-UV analysis of analyte solutions before and after treatment with polymer and calculated as [(peak area before binding-peak area after binding)/peak area before binding] $\times 100 .{ }^{\mathrm{b}}$ Initial concentration $=100 \mathrm{ppm} .{ }^{\mathrm{c}}$ Initial concentration $=500 \mathrm{ppm}$.

\subsubsection{Binding Selectivity of the TMIP 1}

After removing the template from $\mathrm{TMIP}_{1}$, re-binding tests between $\mathrm{BPA}$ and $\mathrm{TMIP}_{1}$ particles (prepared by partial esterification of MIP particles suspended in diethyl ether) were performed using a CE-UV system. Control binding tests were also performed on MIP and NIP particles. To determine the selectivity for BPA in the presence of positively and negatively charged compounds, two different anti-inflammatory drugs were chosen: 
metformin (MF) and diclofenac (DFC). Their chemical structures and $\mathrm{pK}_{\mathrm{a}}$ values are shown in Table 3.2 for comparison with BPA.

Table 3.2 Chemical structures and $\mathrm{pKa}$ values of BPA, DFC and MF.

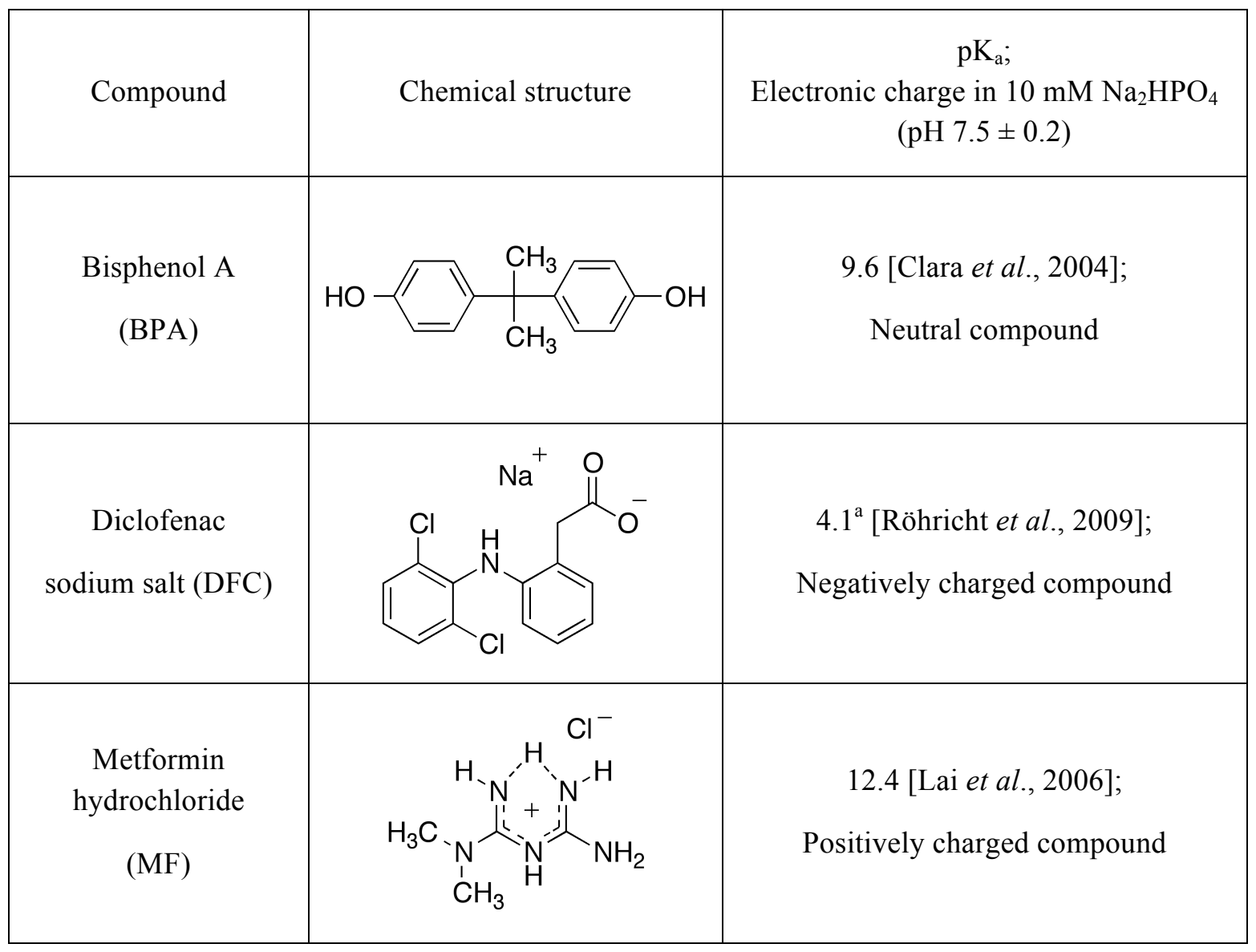

${ }^{\mathrm{a}} \mathrm{pK}_{\mathrm{a}}$ of the corresponding carboxylic acid.

The binding test results for BPA, DFC and MF, both individually and as a mixture, with the four different MAA-based polymers as well as PMMA are summarized in Table 3.3. Examining the individual and mixture results, it is obvious that treatment of the $\mathrm{MIP}_{\mathbf{1}}$ with diazomethane retained or improved the binding of BPA, the target analyte: The binding affinity of BPA to $\mathrm{TMIP}_{1}$ was $98 \% \pm 1 \%$ and $95 \% \pm 1 \%$, respectively, in the analysis of the BPA alone and in the analyte mixture, versus $90 \% \pm 2 \%$ and $94 \% \pm 1 \%$ 
for the $\mathrm{MIP}_{1}$. Not surprisingly, PMMA and the non-imprinted polymethacrylic acid polymer, both with $\left(\mathrm{TNIP}_{1}\right)$ and without $\left(\mathrm{NIP}_{1}\right)$ diazomethane treatment, all afforded substantially lower binding affinities.

It is worth noting that our ethereal diazomethane likely contained a small amount of ethanol and a trace amount of water from the diazomethane preparation and distillation protocol [Sigma-Aldrich Technical Bulletin AL-180, 2007]; however, given the contrasting results of the $\mathrm{TMIP}_{*}$ prepared in a $1: 1$ methanol/THF mixture (TMIP*, Table3.1) versus the TMIP prepared in ether (TMIP ${ }_{1}$, Table 3.3), these small amounts of hydrogen bond donating solvents do not appear to be of significant consequence.

Given that analytical samples will often contain charged species that may interfere with the extraction and analysis of BPA levels; we also opted to assay the binding of a negatively charged species, DFC and a positively charged compound, MF. Once again, $\mathrm{TMIP}_{1}$ proved to be superior to the non-esterified $\mathrm{MIP}_{1}$, affording a lower affinity toward MF, with only $10 \% \pm 1 \%$ and $8 \% \pm 1 \%$ binding individually and in the mixture, respectively, compared with $\mathrm{MIP}_{1}$ values at $56 \% \pm 2 \%$ and $22 \% \pm 2 \%$. This can be rationalized by increased hydrogen bonding of MF with the low affinity sites in the $\mathrm{MIP}_{1}$, as well as electrostatic attraction of MF to carboxylic acid residues that have dissociated to form carboxylate ions. This interpretation is corroborated by the binding affinity values for the $\mathrm{NIP}_{1}$, which is capable of hydrogen-bond donation and electrostatic attraction to $\mathrm{MF}$, and PMMA and $\mathrm{TNIP}_{1}$, which are not. The data in Table 3.4 (vide infra), which, via electrophoretic mobility, orders the different polymers according to their amount of negative charge [DeMaleki et al., 2010], also supports this perspective. 
The negatively charged compound, DFC, displayed binding affinity in the order $\mathrm{MIP}_{1}<$ $\mathrm{TMIP}_{1} \leq \mathrm{NIP}_{1}$. That the MIP 1 has a slightly lower affinity for DFC $(13 \% \pm 1 \%$ versus $16 \% \pm 3 \%$ for $\mathrm{TMIP}_{1}$ in the individual analysis and $7 \% \pm 1 \%$ versus $16 \% \pm 1 \%$ in the mixture) can be explained by $\mathrm{MIP}_{1}$ having a higher level of negative charge (again due to acid dissociation to carboxylate ions, vide infra), whereas the TMIP $_{1}$ has significantly fewer acid residues, thus minimizing charge-charge repulsion. Therefore, blocking the non-specific binding sites via treatment with $\mathrm{CH}_{2} \mathrm{~N}_{2}$ helped reduce MF binding while maintaining both low affinity for DFC and extremely high affinity for BPA - even in the presence of other compounds, the best selectivity for BPA was attained by $\mathrm{TMIP}_{1}$ in comparison to $\mathrm{MIP}_{1}, \mathrm{NIP}_{1}, \mathrm{TNIP}_{1}$ and PMMA. 
Table 3.3 Binding test results for BPA, DFC, MF and mixture $(\mathrm{BPA}+\mathrm{DFC}+\mathrm{MF})$ with polymers.

\begin{tabular}{|c|c|c|c|c|}
\hline Polymer & Target analyte & $\begin{array}{c}\text { Negatively } \\
\text { charged compound }\end{array}$ & $\begin{array}{l}\text { Positively charged } \\
\text { compound }\end{array}$ & Mixture \\
\hline $\mathrm{MIP}_{1}$ & $\mathrm{BPA}=90 \% \pm 2 \%$ & $\mathrm{DFC}=13 \% \pm 1 \%$ & $\mathrm{MF}=56 \% \pm 2 \%$ & $\begin{array}{c}\mathrm{BPA}=94 \% \pm 1 \% \\
\mathrm{DFC}=7 \% \pm 1 \% \\
\mathrm{MF}=22 \% \pm 2 \%\end{array}$ \\
\hline $\mathrm{NIP}_{1}$ & $\mathrm{BPA}=71 \% \pm 5 \%$ & $\mathrm{DFC}=33 \% \pm 2 \%$ & $\mathrm{MF}=65 \% \pm 2 \%$ & $\begin{array}{c}\mathrm{BPA}=55 \% \pm 1 \% \\
\mathrm{DFC}=15 \% \pm 1 \% \\
\mathrm{MF}=37 \% \pm 2 \%\end{array}$ \\
\hline $\mathrm{TMIP}_{1}{ }^{\mathrm{b}}$ & $\mathrm{BPA}=98 \% \pm 1 \%$ & $\mathrm{DFC}=16 \% \pm 3 \%$ & $\mathrm{MF}=10 \% \pm 1 \%$ & $\begin{aligned} \mathrm{BPA} & =95 \% \pm 1 \% \\
\mathrm{DFC} & =16 \% \pm 1 \% \\
\mathrm{MF} & =8 \% \pm 1 \%\end{aligned}$ \\
\hline $\mathrm{TNIP}_{1}^{\mathrm{c}}$ & $\mathrm{BPA}=52 \% \pm 2 \%$ & $\mathrm{DFC}=7 \% \pm 1 \%$ & $\mathrm{MF}=10 \% \pm 1 \%$ & $\begin{array}{c}\mathrm{BPA}=66 \% \pm 3 \% \\
\mathrm{DFC}=7 \% \pm 1 \% \\
\mathrm{MF}=10 \% \pm 1 \%\end{array}$ \\
\hline PMMA & $\mathrm{BPA}=68 \% \pm 1 \%$ & $\mathrm{DFC}=22 \% \pm 2 \%$ & $\mathrm{MF}=11 \% \pm 1 \%$ & $\begin{array}{l}\mathrm{BPA}=58 \% \pm 1 \% \\
\mathrm{DFC}=24 \% \pm 1 \% \\
\mathrm{MF}=18 \% \pm 1 \%\end{array}$ \\
\hline
\end{tabular}

${ }^{a}$ Percentage binding was determined by CE-UV analysis of analyte solutions before and after treatment with polymer and calculated as [(peak area before binding-peak area after binding)/peak area before binding] $\times 100 .{ }^{b} \mathrm{MIP}_{1}$, in the presence of the template BPA, was suspended in diethyl ether during reaction with diazomethane to produce $\mathrm{TMIP}_{1}$. ${ }^{\mathrm{C}} \mathrm{NIP}_{1}$ was suspended in diethyl ether during reaction with diazomethane to produce $\mathrm{TNIP}_{1}$. 


\subsubsection{Electrophoretic Mobility of the Polymers}

The negative or positive charges of $\mathrm{NIP}_{1}, \mathrm{MIP}_{1}, \mathrm{TMIP}_{1}, \mathrm{TNIP}_{1}$ and PMMA particles were determined by CE-UV analysis after they were suspended in BGE with ultrasonication. PMMA was used as a control. The electrophoretic mobility $\mu_{\mathrm{ep}}$ of each polymer was calculated by subtracting the electroosmotic flow $\left(\mu_{\mathrm{eo}}\right)$ of the background electrolyte from the apparent mobility $\mu_{\text {app }}$ [Lai et al., 2010]:

$\mu_{\mathrm{ep}}=\mu_{\mathrm{app}}-\mu_{\mathrm{eo}}$

It can be seen in Table 3.4 that all the five polymers displayed negative electrophoretic mobility values. However, $\mathrm{TMIP}_{1}$, as expected, afforded a decreased amount of negative charge compared to $\mathrm{NIP}_{1}$ and $\mathrm{MIP}_{1}$. This is consistent with a decreasing number of carboxylic acid functional groups, due to their conversion to MMA residues in $\mathrm{TMIP}_{1}$. Critically, $\mathrm{TMIP}_{1}$ also has more negative charge than PMMA and $\mathrm{TNIP}_{1}$, and this is also congruent with our desired partial esterification of the $\mathrm{MIP}_{1}$. In addition, $\mathrm{TNIP}_{1}$, in which we sought to esterify the MAA residues (thus essentially forming a PMMA), closely resembled the mobility of PMMA. 
Table 3.4 Electrophoretic mobility values of $\mathrm{NIP}_{1}, \mathrm{MIP}_{1}, \mathrm{TMIP}_{1}, \mathrm{PMMA}$ and $\mathrm{TNIP}_{1}$

\begin{tabular}{|l|l|}
\hline Polymer & Electrophoretic Mobility $\left(\mathrm{m}^{2} \mathrm{~V}^{-1} \mathrm{~s}^{-1}\right)$ \\
\hline $\mathrm{NIP}_{1}$ & $-2.7 \times 10^{-8}$ \\
\hline $\mathrm{MIP}_{1}$ & $-2.4 \times 10^{-8}$ \\
\hline $\mathrm{TMIP}_{1}^{\mathrm{a}}$ & $-2.0 \times 10^{-8}$ \\
\hline $\mathrm{PMMA}^{\mathrm{s}}$ & $-1.7 \times 10^{-8}$ \\
\hline $\mathrm{TNIP}_{1}{ }^{\mathrm{b}}$ & $-1.5 \times 10^{-8}$ \\
\hline
\end{tabular}

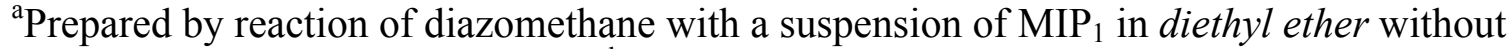
prior removal of the BPA template. ${ }^{b}$ Prepared by reaction of diazomethane with suspension of $\mathrm{NIP}_{1}$ in diethyl ether

\subsubsection{Solid-State ${ }^{13} \mathrm{C}$ NMR}

To further confirm the functional groups or residues in each polymer, we elected to analyze the $\mathrm{NIP}_{1}, \mathrm{MIP}_{1}$ and $\mathrm{TMIP}_{1}$ particles via solid-state ${ }^{13} \mathrm{C}$ NMR spectroscopy. The different spectra (Figure 3.1) - $\mathrm{TMIP}_{1}, \mathrm{NIP}_{1}, \mathrm{MIP}_{1}$ in the presence and absence of the template - show signals for both the EGDMA cross-linker and the MAA residues. These spectra can be compared with a previous study by [Courtois et al., 2006] where ethylene dimethacrylate (EDMA) and MAA were used in their polymer. Figures 3.2 and 3.3 depict the ${ }^{13} \mathrm{C}$ spectra of PMMA and $\mathrm{TNIP}_{1}$, respectively. These data confirm the partial esterification of $\mathrm{TMIP}_{1}$ in two aspects. The first is that a peak in the methyl ester region of the spectrum [Angiolini et al., 2008] is prominently observed between 52.0 and 52.1 ppm in the spectra of $\mathrm{TMIP}_{1}, \mathrm{TNIP}_{1}$, and PMMA, but is absent from the spectra of the unesterified $\mathrm{MIP}_{1}$ and $\mathrm{NIP}_{1}$. While this confirms that methyl esterification did occur in the reaction of $\mathrm{MIP}_{1}$ and NIP 1 with diazomethane to produce $\mathrm{TMIP}_{1}$ and $\mathrm{TNIP}_{1}$, it does not provide any perspective on the degree of esterification. 
In order to gain insight into whether or not $\mathrm{TMIP}_{1}$ was partially or fully esterified during the reaction with diazomethane, we examined the carbonyl signals in the various ${ }^{13} \mathrm{C}$ NMR spectra. The carbonyl signals did indeed vary across our spectra, with signals ranging from 177.3 to $178.2 \mathrm{ppm}$. The trend of the carbonyl peak shifting to a more shielded position when an acid is converted to its corresponding methyl ester is a wellprecedented phenomenon [Breitmaier et al., 1987]. While the EGDMA cross-linker provides a large number of ester carbonyls in all of the polymers, the MIP ${ }_{1}$ and $\mathrm{NIP}_{1}$ still have a significant number of carboxylic acid groups (the 7:8 molar ratio of EGDMA to MAA used should lead to an approximate 14:8 (or 7:4) ester to acid ratio). PMMA, on the other hand, should contain only ester carbonyls and hence yield a more shielded carbonyl signal. This is indeed observed, with PMMA falling at $177.3 \mathrm{ppm}$ and $\mathrm{NIP}_{1}$ and $\mathrm{MIP}_{1}$ (in the absence of the template) at 178.2 and $178.1 \mathrm{ppm}$, respectively. TNIP ${ }_{1}$, which lacked any template binding to mask the acid residues, afforded a carbonyl signal at $177.3 \mathrm{ppm}$, consistent with exhaustive esterification and hence a polymer of nearly identical primary structure to PMMA. This is further underscored by comparison of their full spectra. Critically, at $177.5 \mathrm{ppm}$, the chemical shift of the carbonyl signal of TMIP 1 was intermediate between these two extremes, thus supporting our expectation of partial esterification of the MAA residues (Table 3.5). While the broad nature of signals in solidstate ${ }^{13} \mathrm{C}$ NMR spectra do not afford the opportunity for a more quantitative analysis, this trend of the chemical shifts of the carbonyl peaks is heavily corroborated by our electrophoretic mobility data (Table 3.4). 
Table 3.5 Carbonyl signals in $\mathrm{NIP}_{1}, \mathrm{MIP}_{1}, \mathrm{TMIP}_{1}$, $\mathrm{TNIP}_{1}$, and PMMA.

\begin{tabular}{|l|l|}
\hline Polymer & Carbonyl signal (ppm) \\
\hline NIP $_{1}$ & 178.2 \\
\hline MIP $_{\mathbf{1}}$ & 178.1 \\
\hline TMIP $_{1}$ & 177.5 \\
\hline TNIP $_{1}$ & 177.2 \\
\hline PMMA & 177.2 \\
\hline
\end{tabular}

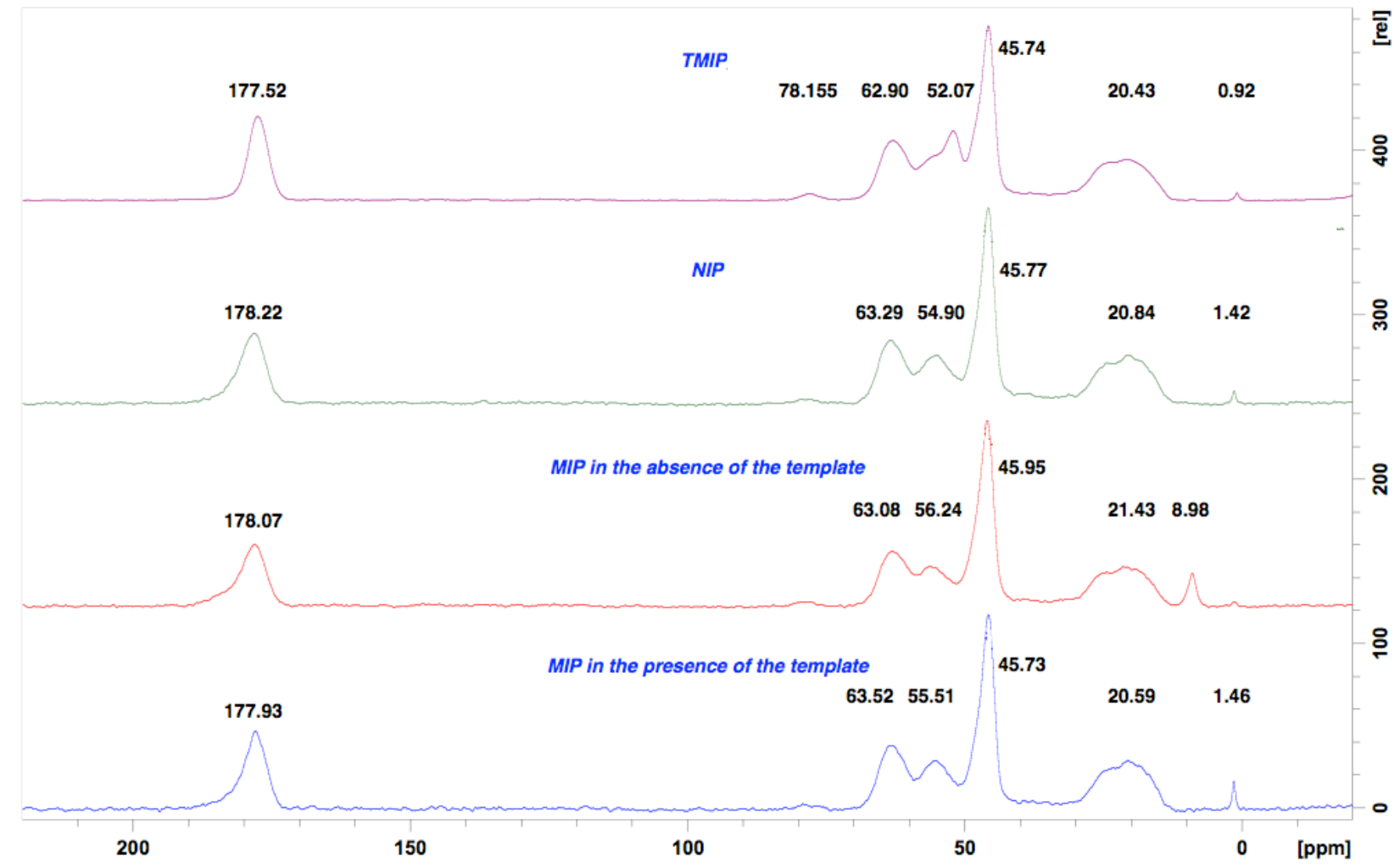

Figure 3.1 ${ }^{13} \mathrm{C}$ NMR spectra of $\mathrm{TMIP}_{1}, \mathrm{NIP}_{1}, \mathrm{MIP}_{1}$ without BPA and MIP 1 with BPA. 


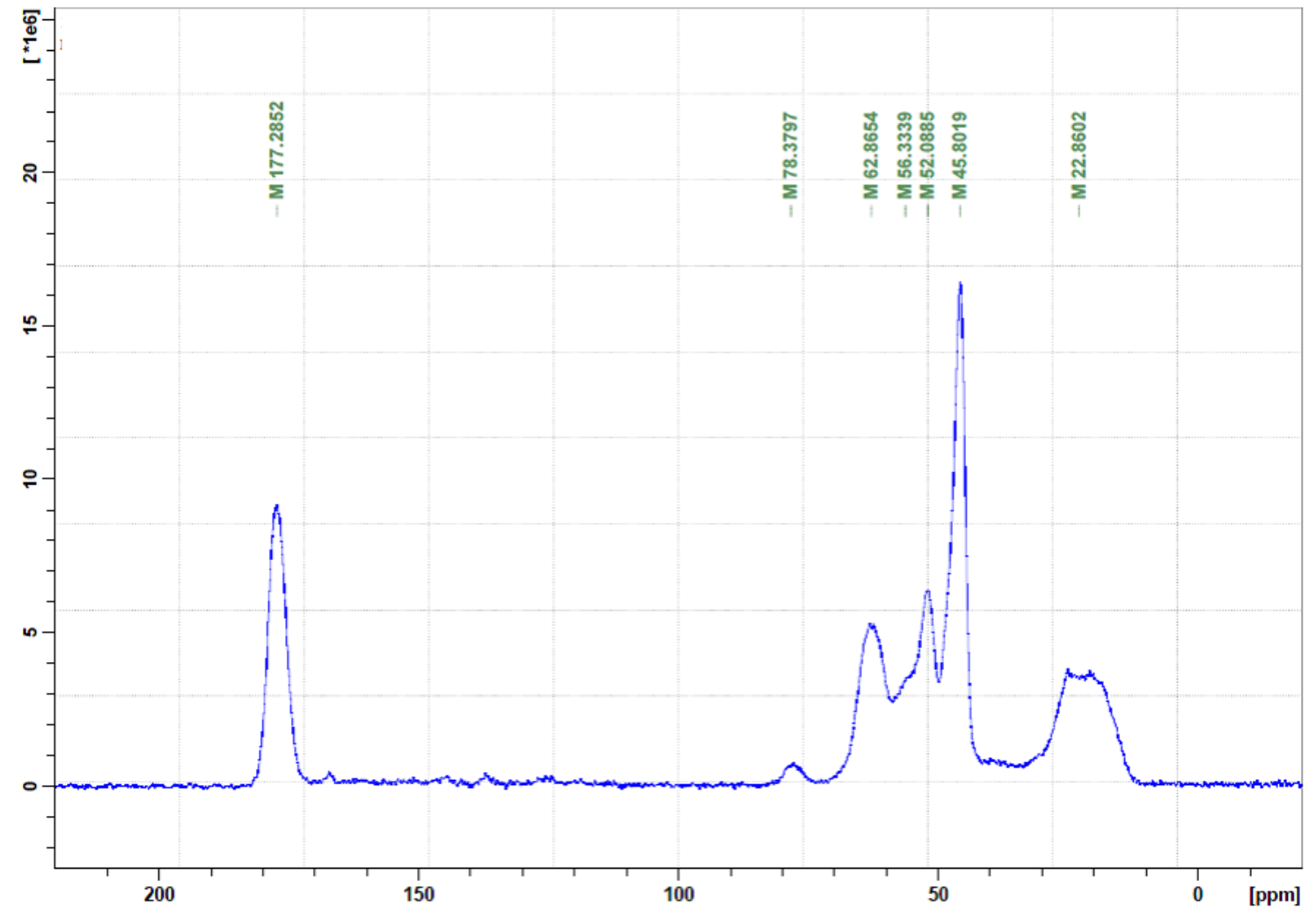

Figure 3.2 ${ }^{13} \mathrm{C}$ NMR spectrum of PMMA.

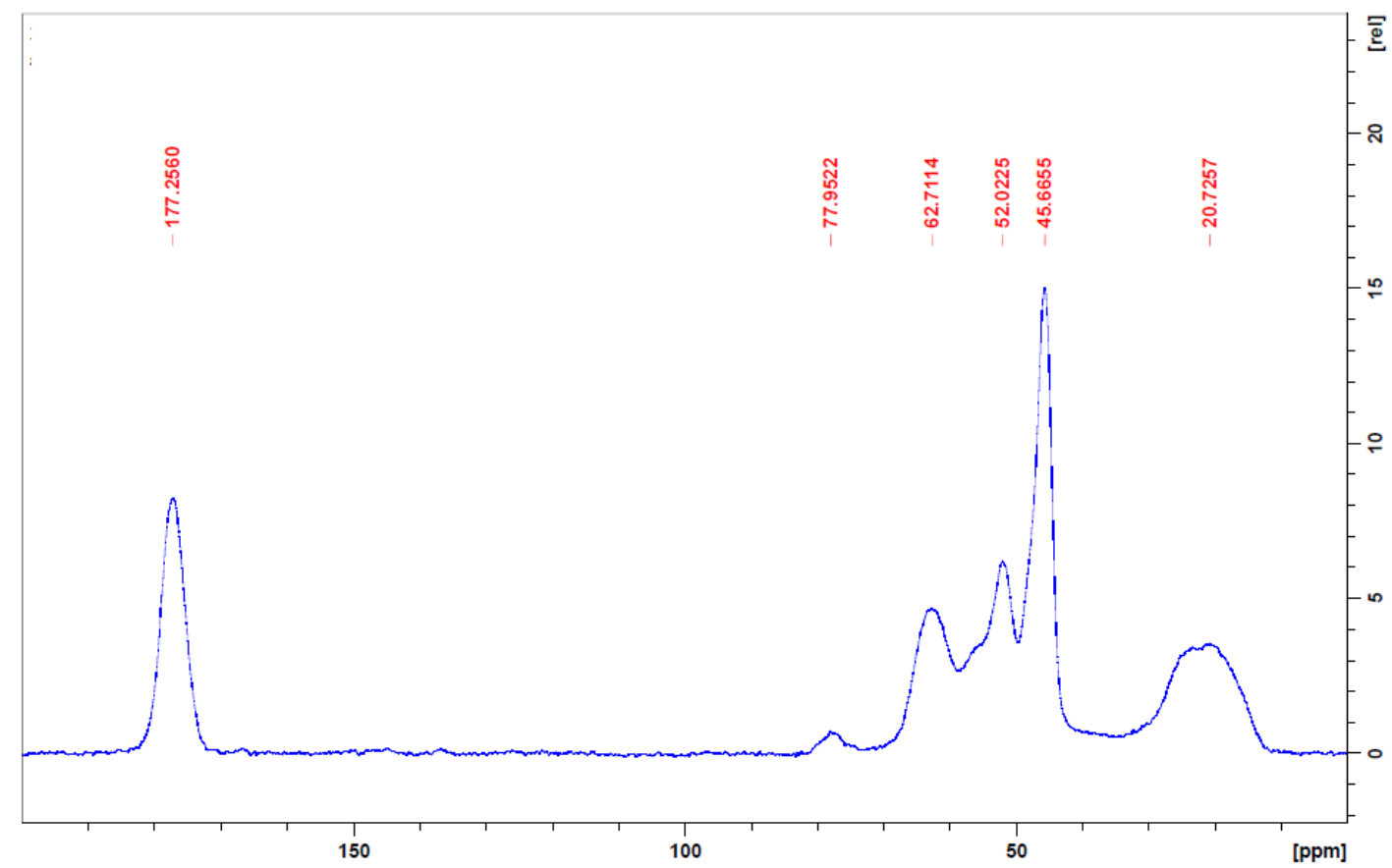

Figure 3.3 ${ }^{13} \mathrm{C}$ NMR spectrum of TNIP 


\subsection{Optimization of Template to Functional Monomer Molar Ratio}

Approach

The molar ratio of BPA to MAA was modified in an attempt to reduce or eliminate nonspecific binding sites in the MIP. This straightforward approach could be an effective method to enhance the selectivity of MIP toward BPA without the use of diazomethane treatment. All TMIP, MIP, TNIP and NIP particles were tested for their BPA selectivity, using DFC and MF as negatively and positively charged interferents, respectively. As the molar ratio of BPA to MAA increases, the binding selectivity of MIP for BPA is enhanced, as can be seen in Table 3.6. DFC exhibits slightly higher affinity towards $\mathrm{MIP}_{8}, \mathrm{MIP}_{9}$ and $\mathrm{MIP}_{10}$ than the other MIPs, probably due to stronger hydrophobic interaction with these former MIP particles as the ratio of MAA to EGDMA decreases, and the polymer becomes less negatively charged. Obviously, MIP $_{10}$ shows the lowest binding affinity toward metformin (MF), both individually and in mixture, which, in conjunction with the electrophoretic mobility data, indicates that this polymer has the lowest negative charge among the MIPs. All percent binding results of BPA, DFC and MF (individually and in mixture), as well as electrophoretic mobility, are presented in Figure 3.4 for each MIP prepared with a different BPA mole \%. A significant decrease in $\%$ binding for MF and a slight increase in \% binding for DFC are clearly seen when going from $\mathrm{MIP}_{2}$ to $\mathrm{MIP}_{10}$, all of which exhibit a constant $99 \%$ binding for BPA. 
Table 3.6 Percent binding results for BPA, DFC and MF (individually and in mixture) and electrophoretic mobility for BPA MIPs.

\begin{tabular}{|c|c|c|c|c|c|c|c|c|}
\hline \multirow{2}{*}{$\begin{array}{c}\text { MIPs } \\
\text { (BPA:MAA:EGDMA } \\
\text { molar ratio) }\end{array}$} & \multirow{2}{*}{$\begin{array}{c}\text { Mole \% } \\
\text { BPA in } \\
\text { MIP } \\
\text { Preparation }\end{array}$} & \multicolumn{3}{|c|}{$\%$ Binding (individual) } & \multicolumn{3}{|c|}{$\%$ Binding (mixture) } & \multirow{2}{*}{$\begin{array}{l}\text { Electrophoretic } \\
\text { mobility } \\
\left(10^{-9} \mathrm{~m}^{2} \mathrm{~V}^{-1} \mathrm{~s}^{-1}\right)\end{array}$} \\
\hline & & BPA & $\mathrm{DFC}$ & $\mathrm{MF}$ & $\mathrm{BPA}$ & $\mathrm{DFC}$ & MF & \\
\hline $\mathrm{MIP}_{2}(1: 9: 7)$ & 6 & $99 \pm 1$ & $13 \pm 1$ & $84 \pm 1$ & $99 \pm 1$ & $11 \pm 1$ & $81 \pm 1$ & 24 \\
\hline $\mathrm{MIP}_{3}(2: 8: 7)$ & 12 & $99 \pm 1$ & $15 \pm 1$ & $69 \pm 1$ & $99 \pm 1$ & $13 \pm 1$ & $70 \pm 1$ & 26 \\
\hline $\mathrm{MIP}_{4}(3: 7: 7)$ & 18 & $99 \pm 1$ & $16 \pm 1$ & $69 \pm 1$ & $99 \pm 1$ & $11 \pm 1$ & $64 \pm 1$ & 27 \\
\hline $\mathrm{MIP}_{5}(4: 6: 7)$ & 24 & $99 \pm 1$ & $13 \pm 1$ & $67 \pm 1$ & $99 \pm 1$ & $12 \pm 1$ & $63 \pm 1$ & 27 \\
\hline $\mathrm{MIP}_{6}(5: 5: 7)$ & 29 & $99 \pm 1$ & $17 \pm 1$ & $61 \pm 1$ & $99 \pm 1$ & $17 \pm 1$ & $61 \pm 1$ & 27 \\
\hline $\operatorname{MIP}_{7}(6: 4: 7)$ & 35 & $99 \pm 1$ & $19 \pm 1$ & $60 \pm 1$ & $99 \pm 1$ & $15 \pm 1$ & $57 \pm 1$ & 28 \\
\hline $\mathrm{MIP}_{8}(7: 3: 7)$ & 41 & $99 \pm 1$ & $29 \pm 1$ & $59 \pm 1$ & $99 \pm 1$ & $27 \pm 1$ & $55 \pm 1$ & 30 \\
\hline $\mathrm{MIP}_{9}(8: 2: 7)$ & 47 & $99 \pm 1$ & $29 \pm 1$ & $51 \pm 1$ & $99 \pm 1$ & $28 \pm 3$ & $48 \pm 1$ & 30 \\
\hline $\operatorname{MIP}_{10}(9: 1: 7)$ & 53 & $99 \pm 1$ & $25 \pm 1$ & $32 \pm 1$ & $99 \pm 1$ & $25 \pm 1$ & $33 \pm 1$ & 30 \\
\hline
\end{tabular}




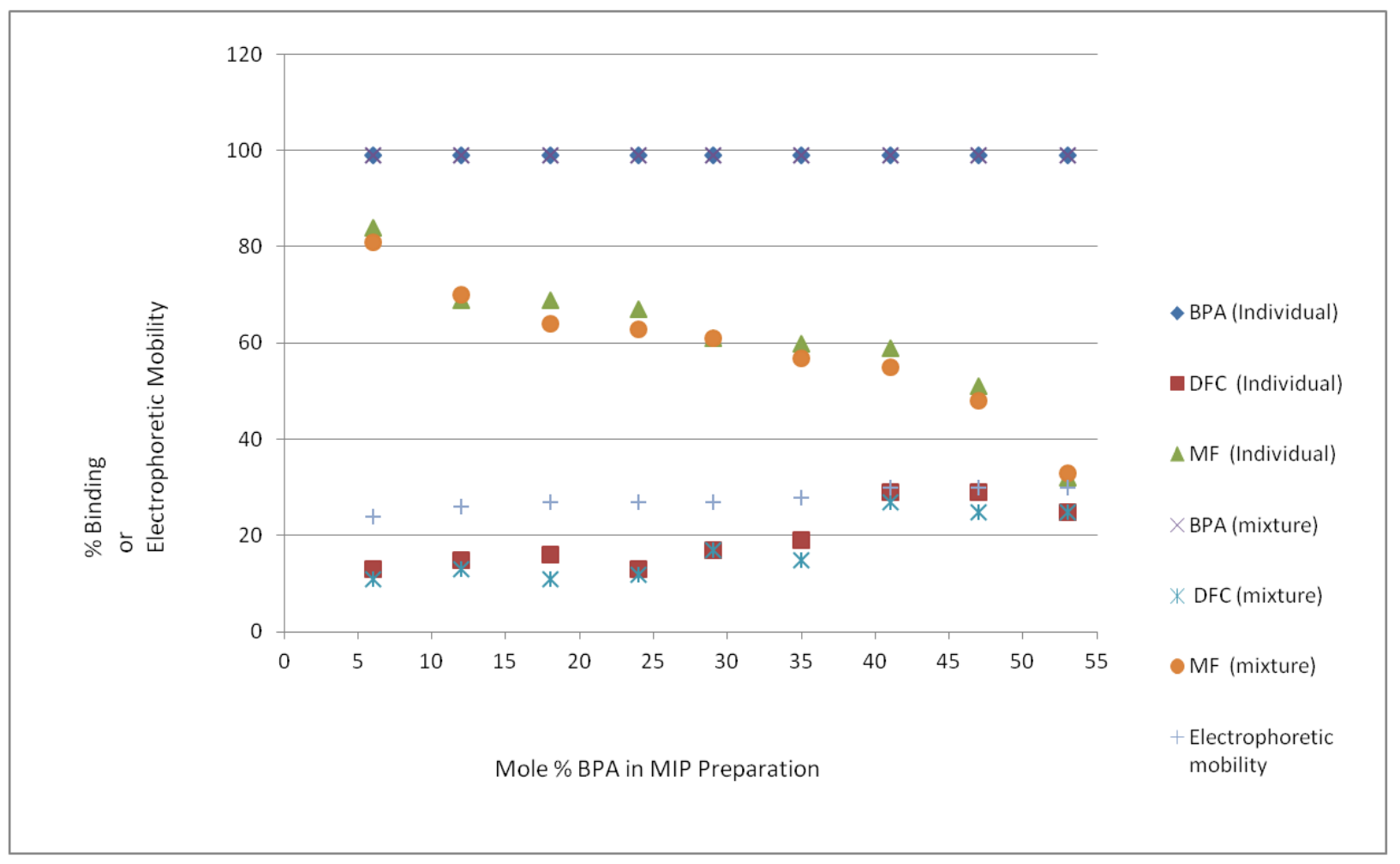

Figure 3.4 Percent binding results of BPA, DFC and MF (individually and in mixture) and electrophoretic mobility for each MIP prepared with a different mole \% of BPA. For clarity, error bars are represented by the size of symbols.

For comparison purposes, the study tested the binding affinity of BPA (individually and in the three-component mixture) toward NIPs. When the ratio of MAA to EGDMA decreased upon going from $\mathrm{NIP}_{2}$ to $\mathrm{NIP}_{10}$, as shown in Table 3.7, the binding affinity of DFC and BPA toward the NIPs increased due to elevated hydrophobic interactions and in the case of DFC, decreased electrostatic repulsion - that is the same trend as with MIPs. The binding affinity of MF decreased from $70 \% \pm 1 \%$ to $50 \% \pm 1 \%$ individually and from $73 \% \pm 1 \%$ to $56 \% \pm 1 \%$ in mixture. While the direction of this trend in consistent with the results in MIPs, the magnitude is far less pronounced - these decreases observed with the NIPs were neither so significant in range (from highest to lowest) nor related to 
the electrophoretic mobility values (which indicate a fairly constant electronic charge to particle size ratio for all of the polymers). The apparently higher values of electrophoretic mobility for NIPs do not necessarily mean higher electronic charges, but rather smaller sizes than MIPs, due to the absence of imprinted cavities in the NIPs (vide infra).

Table 3.7 Percent binding results for BPA, DFC and MF (individually or in mixture) and electrophoretic mobility for NIPs.

\begin{tabular}{|c|c|c|c|c|c|c|c|}
\hline \multirow{2}{*}{$\begin{array}{c}\text { NIPs } \\
\text { (MAA:EGDMA } \\
\text { molar ratio) }\end{array}$} & \multicolumn{2}{|c|}{$\%$ Binding (individual) } & \multicolumn{2}{|c|}{$\%$ Binding (mixture) } & $\begin{array}{c}\text { Electrophoretic } \\
\text { mobility }\end{array}$ \\
\cline { 2 - 7 } & BPA & DFC & MF & BPA & DFC & MF & \multirow{2}{*}{$\left(10^{-9} \mathrm{~m}^{2} \mathrm{~V}^{-1} \mathrm{~s}^{-1}\right)$} \\
\hline $\mathrm{NIP}_{1}(9: 7)$ & $80 \pm 1$ & $25 \pm 1$ & $70 \pm 1$ & $75 \pm 1$ & $25 \pm 1$ & $73 \pm 1$ & 29 \\
\hline $\mathrm{NIP}_{2}(8: 7)$ & $88 \pm 1$ & $26 \pm 1$ & $73 \pm 1$ & $75 \pm 1$ & $18 \pm 2$ & $67 \pm 1$ & 27 \\
\hline $\mathrm{NIP}_{3}(7: 7)$ & $91 \pm 1$ & $29 \pm 1$ & $71 \pm 1$ & $92 \pm 1$ & $33 \pm 2$ & $74 \pm 1$ & 28 \\
\hline $\mathrm{NIP}_{4}(6: 7)$ & $92 \pm 1$ & $31 \pm 1$ & $62 \pm 1$ & $92 \pm 1$ & $25 \pm 2$ & $60 \pm 1$ & 29 \\
\hline $\mathrm{NIP}_{5}(5: 7)$ & $91 \pm 1$ & $29 \pm 2$ & $75 \pm 1$ & $96 \pm 1$ & $32 \pm 1$ & $62 \pm 1$ & 29 \\
\hline $\mathrm{NIP}_{6}(4: 7)$ & $90 \pm 1$ & $25 \pm 1$ & $69 \pm 1$ & $97 \pm 2$ & $28 \pm 3$ & $58 \pm 1$ & 28 \\
\hline $\mathrm{NIP}_{7}(3: 7)$ & $99 \pm 1$ & $32 \pm 1$ & $55 \pm 2$ & $99 \pm 1$ & $38 \pm 2$ & $58 \pm 1$ & 28 \\
\hline $\mathrm{NIP}_{8}(2: 7)$ & $99 \pm 1$ & $37 \pm 1$ & $58 \pm 1$ & $99 \pm 1$ & $39 \pm 1$ & $54 \pm 1$ & 28 \\
\hline $\mathrm{NIP}_{9}(1: 7)$ & $96 \pm 1$ & $40 \pm 1$ & $50 \pm 1$ & $99 \pm 1$ & $43 \pm 1$ & $56 \pm 1$ & 28 \\
\hline
\end{tabular}




\subsubsection{Esterification of MIPs with Diazomethane}

To enhance selective binding with the target analyte molecules, diazomethane treatment was chosen as an alternative or combined approach to reduce or eliminate non-specific binding sites in the MIPs [Greene et al., 2005; Umpleby et al., 2001]. In Table 3.8, all TMIPs showed diminished binding affinity toward MF, a positively charged compound. In particular, $\mathrm{TMIP}_{2}$ revealed the lowest binding affinity toward $\mathrm{MF}$, at $9 \% \pm 1 \%$ individually and $10 \% \pm 1 \%$ in mixture. Unlike our MIPs, BPA binding affinity toward $\mathrm{TMIP}_{2}$ in the three-component mixture shows a slightly lower binding affinity of $91 \% \pm$ $1 \%$. As the molar ratio of BPA is increased in Table 3.8, the binding affinity of TMIPs is enhanced up to $99 \%$ (both individually and in the mixture for $\mathrm{TMIP}_{5}$ to $\mathrm{TMIP}_{10}$ ) presumably as a result of an increase in the number of specific binding sites. All TMIPs show less binding affinity than MIPs toward DFC, particularly $\mathrm{TMIP}_{2}$ at only $6 \% \pm 1 \%$ individually and $8 \% \pm 1 \%$ in mixture. TMIPs also show significantly less binding affinity toward MF when compared to MIPs, which indicates a decrease of electrostatic interactions between MF and the polymer. Comparing the binding affinity of MF in the three-compound mixture, $\mathrm{TMIP}_{2}$ demonstrates the most dramatic decrease, dropping to $10 \%$ from $73 \%$ for $\mathrm{MIP}_{2}$. All binding results are presented in Figure 3.5 for each TMIP. The separation of BPA data points at the top from those for DFC and MF near the bottom is a very distinctive and impressive illustration of the high selectivity offered by TMIPs toward the target analyte. 
Table 3.8 Percent binding results for BPA, DFC and MF (individually or in mixture) and electrophoretic mobility for TMIPs.

\begin{tabular}{|c|c|c|c|c|c|c|c|c|}
\hline \multirow{2}{*}{$\begin{array}{c}\text { TMIPs } \\
\text { (BPA:MAA: } \\
\text { EGDMA } \\
\text { molar ratio) }\end{array}$} & \multirow{2}{*}{$\begin{array}{c}\text { Mole } \\
\% \\
\text { BPA in } \\
\text { MIP } \\
\text { Prepar- } \\
\text { ation }\end{array}$} & \multicolumn{3}{|c|}{ \% Binding (individual) } & \multicolumn{3}{|c|}{$\%$ Binding (mixture) } & \multirow{2}{*}{$\begin{array}{c}\text { Electrophoreti } \\
\mathrm{c} \\
\text { mobility } \\
\left(10^{-9} \mathrm{~m}^{2} \mathrm{~V}^{-1} \mathrm{~s}^{-1}\right)\end{array}$} \\
\hline & & BPA & DFC & MF & BPA & $\mathrm{DFC}$ & MF & \\
\hline $\mathrm{TMIP}_{2}(1: 9: 7)$ & 6 & $99 \pm 1$ & $6 \pm 1$ & $9 \pm 1$ & $91 \pm 3$ & $8 \pm 1$ & $10 \pm 1$ & 20 \\
\hline $\mathrm{TMIP}_{3}(2: 8: 7)$ & 12 & $99 \pm 1$ & $12 \pm 1$ & $19 \pm 1$ & $95 \pm 1$ & $12 \pm 1$ & $22 \pm 1$ & 24 \\
\hline $\mathrm{TMIP}_{4}(3: 7: 7)$ & 18 & $99 \pm 1$ & $12 \pm 1$ & $23 \pm 1$ & $92 \pm 2$ & $11 \pm 1$ & $21 \pm 1$ & 23 \\
\hline $\mathrm{TMIP}_{5}(4: 6: 7)$ & 24 & $99 \pm 1$ & $12 \pm 1$ & $22 \pm 1$ & $99 \pm 1$ & $11 \pm 1$ & $20 \pm 1$ & 20 \\
\hline $\operatorname{TMIP}_{6}(5: 5: 7)$ & 29 & $99 \pm 1$ & $11 \pm 1$ & $20 \pm 1$ & $99 \pm 1$ & $15 \pm 1$ & $25 \pm 1$ & 21 \\
\hline $\mathrm{TMIP}_{7}(6: 4: 7)$ & 35 & $99 \pm 1$ & $20 \pm 1$ & $13 \pm 1$ & $99 \pm 1$ & $15 \pm 1$ & $18 \pm 1$ & 21 \\
\hline $\mathrm{TMIP}_{8}(7: 3: 7)$ & 41 & $99 \pm 1$ & $23 \pm 1$ & $15 \pm 1$ & $99 \pm 1$ & $17 \pm 1$ & $18 \pm 1$ & 20 \\
\hline $\mathrm{TMIP}_{9}(8: 2: 7)$ & 47 & $99 \pm 1$ & $21 \pm 2$ & $14 \pm 1$ & $99 \pm 1$ & $20 \pm 2$ & $14 \pm 1$ & 20 \\
\hline $\begin{array}{l}\text { TMIP }_{10} \\
(9: 1: 7)\end{array}$ & 53 & $99 \pm 1$ & $20 \pm 1$ & $14 \pm 1$ & $99 \pm 1$ & $17 \pm 1$ & $13 \pm 1$ & 20 \\
\hline
\end{tabular}




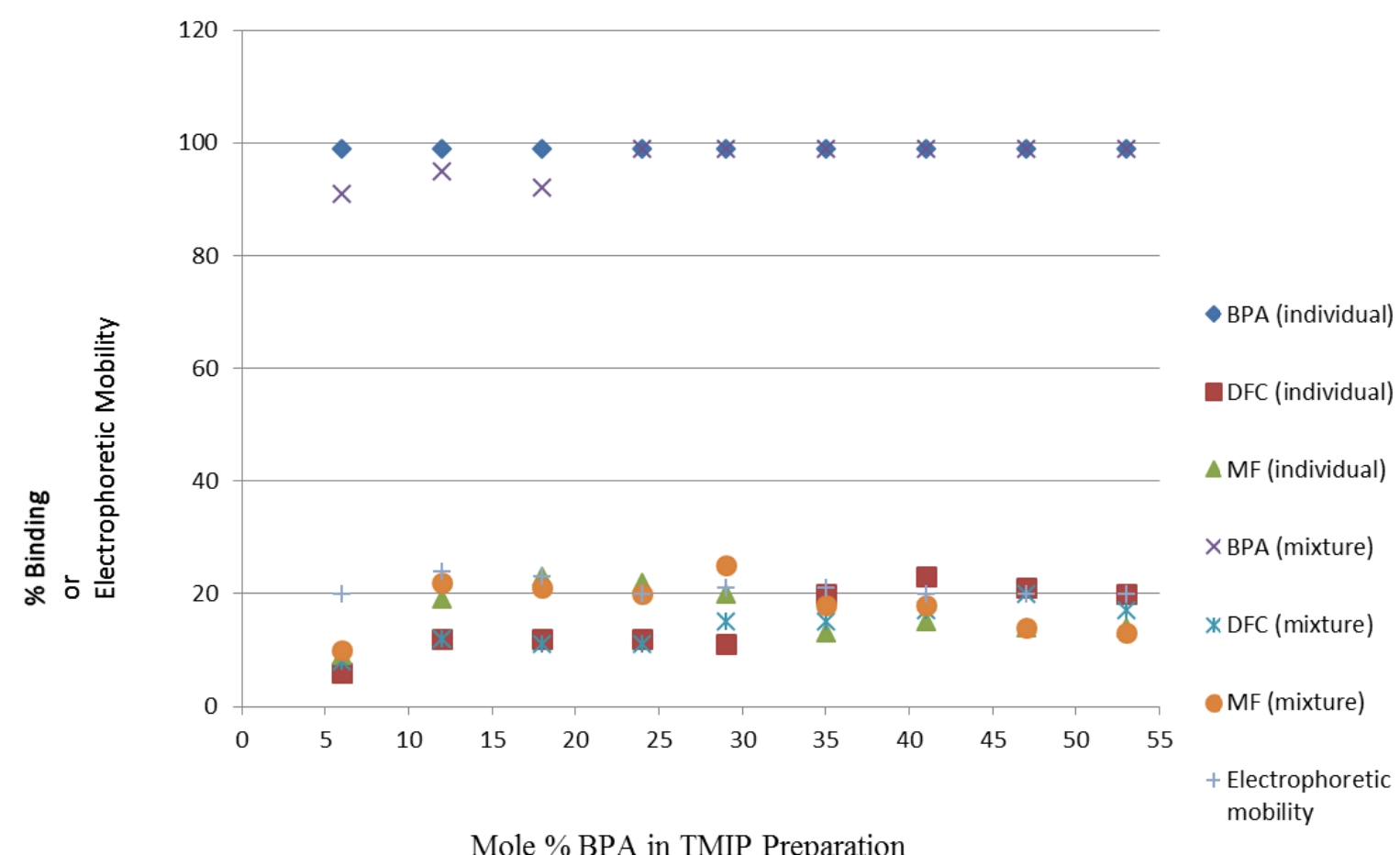

Figure 3.5 Percent binding results of BPA, DFC and MF (individually and in mixture) and electrophoretic mobility for each TMIP prepared with a different mole \% of BPA. For clarity, error bars are represented by the size of symbols.

In comparison with TMIPs, TNIPs show higher non-specific binding affinities toward MF and DFC (Table 3.9). Interestingly, many TNIPs also have a higher binding affinity toward BPA than NIPs. One plausible explanation is that the ester functional group affords stronger interaction with BPA than the carboxylate functional group. Apparently, MAA is not necessarily the functional monomer, but EGDMA is. This is an important finding in the sense that, by converting acids into esters, the number of specific binding sites for BPA was increased in all TMIPs. This would also explain why $\mathrm{TMIP}_{10}$ performed better than $\mathrm{TMIP}_{2}$ in binding with $\mathrm{BPA}$ despite a decrease of the MAA monomer molar ratio from 9 (for $\mathrm{TMIP}_{2}$ ) to 1 (for $\mathrm{TMIP}_{10}$ ). Efforts to make the 10:0:7 
BPA: MAA: EGDMA polymer were to no avail because the particles could not be uniformly dispersed in water samples to perform consistent binding tests.

Table 3.9 Percent binding results for BPA, DFC and MF (individually or in mixture) and electrophoretic mobility for various TNIPs.

\begin{tabular}{|c|c|c|c|c|c|c|c|}
\hline \multirow{2}{*}{$\begin{array}{c}\text { TNIPs } \\
\text { (MAA:EGDMA } \\
\text { molar ratio) }\end{array}$} & \multicolumn{3}{|c|}{ \% Binding (individual) } & \multicolumn{3}{|c|}{$\%$ Binding (mixture) } & \multirow{2}{*}{$\begin{array}{l}\text { Electrophoretic } \\
\text { mobility } \\
\left(10^{-9} \mathrm{~m}^{2} \mathrm{~V}^{-1} \mathrm{~s}^{-1}\right)\end{array}$} \\
\hline & BPA & DFC & MF & BPA & $\mathrm{DFC}$ & MF & \\
\hline $\mathrm{TNIP}_{2}(9: 7)$ & $99 \pm 1$ & $18 \pm 1$ & $54 \pm 1$ & $99 \pm 1$ & $14 \pm 1$ & $41 \pm 1$ & 25 \\
\hline $\mathrm{TNIP}_{3}(8: 7)$ & $99 \pm 1$ & $10 \pm 1$ & $27 \pm 1$ & $90 \pm 1$ & $14 \pm 1$ & $27 \pm 1$ & 24 \\
\hline $\mathrm{TNIP}_{4}(7: 7)$ & $99 \pm 1$ & $16 \pm 1$ & $38 \pm 1$ & $90 \pm 1$ & $16 \pm 1$ & $30 \pm 1$ & 24 \\
\hline $\mathrm{TNIP}_{5}(6: 7)$ & $99 \pm 1$ & $17 \pm 1$ & $30 \pm 1$ & $99 \pm 1$ & $19 \pm 1$ & $26 \pm 1$ & 23 \\
\hline $\operatorname{TNIP}_{6}(5: 7)$ & $99 \pm 1$ & $19 \pm 2$ & $17 \pm 1$ & $99 \pm 1$ & $40 \pm 1$ & $36 \pm 1$ & 24 \\
\hline $\mathrm{TNIP}_{7}(4: 7)$ & $99 \pm 1$ & $26 \pm 1$ & $30 \pm 1$ & $99 \pm 1$ & $26 \pm 1$ & $16 \pm 1$ & 24 \\
\hline $\mathrm{TNIP}_{8}(3: 7)$ & $99 \pm 1$ & $24 \pm 1$ & $29 \pm 1$ & $99 \pm 1$ & $37 \pm 1$ & $22 \pm 1$ & 24 \\
\hline $\mathrm{TNIP}_{9}(2: 7)$ & $99 \pm 1$ & $36 \pm 1$ & $27 \pm 1$ & $99 \pm 1$ & $30 \pm 1$ & $20 \pm 1$ & 23 \\
\hline $\operatorname{TNIP}_{10}(1: 7)$ & $99 \pm 1$ & $45 \pm 1$ & $31 \pm 1$ & $99 \pm 1$ & $54 \pm 1$ & $30 \pm 1$ & 22 \\
\hline
\end{tabular}




\subsubsection{Non-specific Binding Properties}

The non-specific binding properties of MIP, NIP, TMIP and TNIP particles were further investigated by CE-UV analysis of baclofen (BFN), which is a zwitterionic derivative of $\gamma$-aminobutyric acid and is primarily used to treat spastic movement disorders. Although its $\mathrm{pK}_{\mathrm{b}}$ is 3.9 and its $\mathrm{pK}_{\mathrm{a}}$ is 9.8 [Drug bank, 2005], the BFN peak was not be separable from the BPA peak and hence appeared to be a neutral compound. As summarized in Table 3.10, the binding results for BFN are unexpected - significantly lower than those discussed above for BPA and fairly similar to those for diclofenac sodium. It is well known that BPA has a $\mathrm{pK}_{\mathrm{a}}$ value between 9.6 and 11.3 [Bing-zhi et al., 2010] and an octanol-water partitioning coefficient $\left(\mathrm{K}_{\mathrm{ow}}\right)$ value of 103.4 [Cousins et al., 2002]. The latter property indicates that BPA is a significantly more hydrophobic compound than BFN, which has a log partition coefficient (log P) value of - $0.80 \pm 0.02$ [Drug bank, 2005] or a partition coefficient $(\mathrm{P})$ value of 0.16 . Figure 3.6 presents the percent binding results of BFN for each MIP and TMIP prepared with a different mole $\%$ of BPA. Despite the scatter of data points between $5 \%$ binding and $20 \%$ binding, BFN behaves similarly to DFC (see Figure 3.4 and Figure 3.5), which has a $\mathrm{pK}_{\mathrm{a}}$ value of 4.0 [Sandoz Inc., 2007] and a larger molecular size. While their non-specific binding mechanisms (including hydrophobic and ionic interactions) may appear challenging at this time, it is definitely worthy of more systematic investigation. 
Table 3.10 Percent binding results for BFN (individually) with various MIPs, NIPs, TMIPs and TNIPs.

\begin{tabular}{|c|c|c|c|}
\hline & B & $\begin{array}{l}\text { clofen } \\
\text { g (individual) }\end{array}$ & \\
\hline MIPs & NIPs & TMIPs & TNIPs \\
\hline $\mathrm{MIP}_{2}=13 \pm 3$ & $\mathrm{NIP}_{2}=14 \pm 1$ & $\mathrm{TMIP}_{2}=5 \pm 2$ & $\mathrm{TNIP}_{2}=8 \pm 3$ \\
\hline $\mathrm{MIP}_{3}=7 \pm 3$ & $\mathrm{NIP}_{3}=16 \pm 2$ & $\mathrm{TMIP}_{3}=12 \pm 2$ & $\mathrm{TNIP}_{3}=10 \pm 3$ \\
\hline $\mathrm{MIP}_{4}=7 \pm 3$ & $\mathrm{NIP}_{4}=16 \pm 2$ & $\mathrm{TMIP}_{4}=14 \pm 2$ & $\mathrm{TNIP}_{4}=7 \pm 2$ \\
\hline $\mathrm{MIP}_{5}=6 \pm 3$ & $\mathrm{NIP}_{5}=17 \pm 2$ & $\mathrm{TMIP}_{5}=10 \pm 1$ & $\mathrm{TNIP}_{5}=4 \pm 3$ \\
\hline $\mathrm{MIP}_{6}=10 \pm 2$ & $\mathrm{NIP}_{6}=16 \pm 2$ & $\mathrm{TMIP}_{6}=7 \pm 3$ & $\mathrm{TNIP}_{6}=4 \pm 3$ \\
\hline $\mathrm{MIP}_{7=} 10 \pm 2$ & $\mathrm{NIP}_{7}=10 \pm 3$ & $\mathrm{TMIP}_{7}=10 \pm 2$ & $\mathrm{TNIP}_{7}=6 \pm 2$ \\
\hline $\mathrm{MIP}_{8}=14 \pm 2$ & $\mathrm{NIP}_{8}=7 \pm 2$ & $\mathrm{TMIP}_{8}=8 \pm 2$ & $\mathrm{TNIP}_{8}=6 \pm 3$ \\
\hline $\mathrm{MIP}_{9}=10 \pm 1$ & $\mathrm{NIP}_{9}=7 \pm 3$ & $\mathrm{TMIP}_{9}=7 \pm 2$ & $\mathrm{TNIP}_{9}=8 \pm 3$ \\
\hline $\mathrm{MIP}_{10}=10 \pm 1$ & $\mathrm{NIP}_{10}=9 \pm 2$ & $\mathrm{TMIP}_{10}=10 \pm 2$ & $\mathrm{TNIP}_{10}=12 \pm 3$ \\
\hline
\end{tabular}




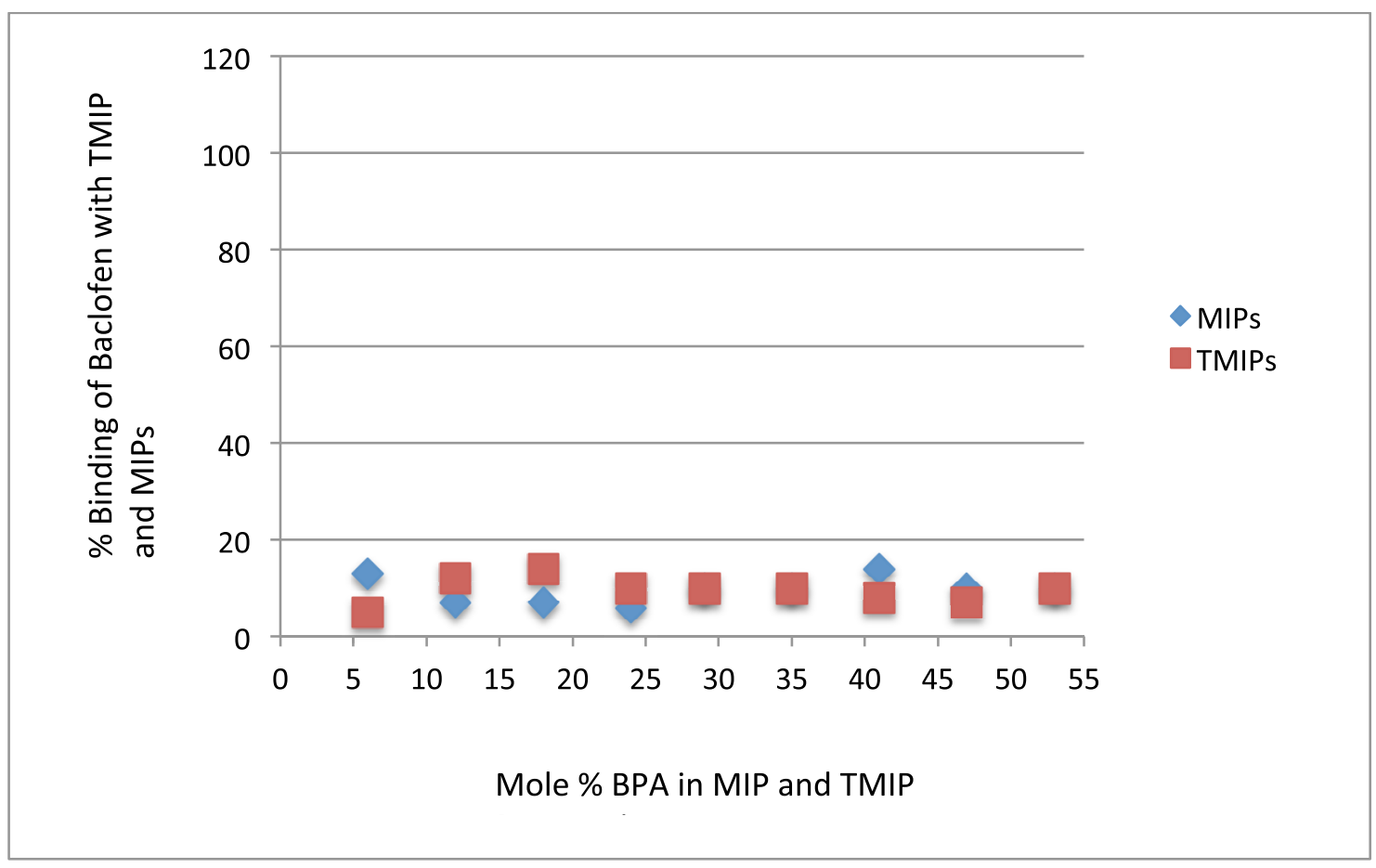

Figure 3.6 Percent binding results of BFN (individually) for each MIP and TMIP prepared with a different mole $\%$ BPA. For clarity, error bars are represented by the size of symbols.

In order to gain a better understanding of the non-specific binding mechanisms, highperformance liquid chromatography with UV detection was used to analyze standard solutions of BFN, BPA, DFC and MF (individually or in mixture) on a reverse-phase column. The retention time of each organic compound, analyzed individually as summarised in Table 3.11, would serve as a direct indication of its hydrophobicity. As illustrated in Figure 3.7, their order of elution was MF first, DFC second, BFN third, and BPA last. This indicated that MF is the least hydrophobic and BPA is the most hydrophobic of the four compounds. The fact that the least hydrophobic compound was able to bind non-specifically with the MIPs (from $32 \%$ to $84 \%$ in Figure 1 and Table 2) emphasizes a real need to perform SSCM on the MIPs by treating them with diazomethane. Apparently, moderately hydrophobic compounds like BFN and DFC bind 
non-specifically with the MIPs not more than 29\% (see Figure 3.4 and Table 3.6) whether they are zwitterionic or negatively charged in aqueous samples. Site-selective chemical modification with diazomethane, however, can decrease their non-specific bindings down to $23 \%$ (see Figure 3.5 and Table 3.4 ).

Table 3.11 HPLC-UV peak heights, peak areas and retention times for BFN, BPA, DFC and MF.

\begin{tabular}{|c|c|c|c|}
\hline $\begin{array}{c}\text { Concentration } \\
(\mathrm{ppm})\end{array}$ & Peak area & Peak height & $\begin{array}{c}\text { Retention time } \\
\text { (min) }\end{array}$ \\
\hline MF (200) & 679024 & 38547 & 2.5 \\
\hline DFC (200) & 49417648 & 1205569 & 3.6 \\
\hline BFN (200) & 12274367 & 417392 & 4.1 \\
\hline BPA (100) & 32571576 & 1487642 & 6.6 \\
\hline
\end{tabular}




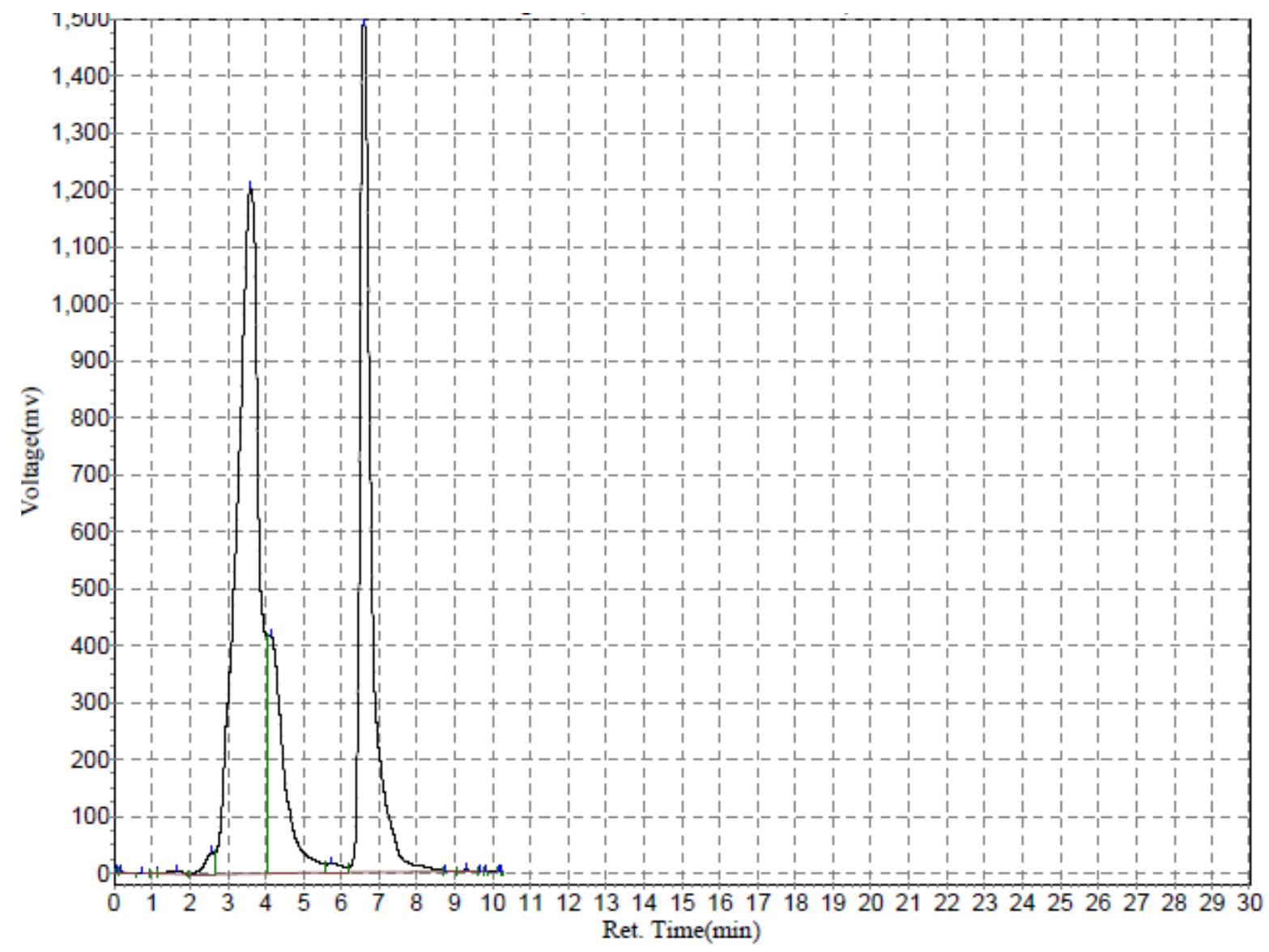

Figure 3.7 HPLC analysis of a mixture of BPA (100 ppm) at $2.5 \mathrm{~min}$, BFN (200 ppm) at $3.6 \mathrm{~min}, \mathrm{DFC}(200 \mathrm{ppm})$ at $4.1 \mathrm{~min}$ and $\mathrm{MF}(200 \mathrm{ppm})$ at $6.6 \mathrm{~min}$. Mobile phase: $\mathrm{CH}_{3} \mathrm{CN} / \mathrm{MeOH} / \mathrm{DDW}(1: 1: 1 \mathrm{v} / \mathrm{v})$; flow rate, $0.8 \mathrm{~mL} / \mathrm{min}$; UV detection at $200 \mathrm{~nm}$.

\subsubsection{Average Size of MIPs and NIPs vs TMIPs and NTIPs}

The average sizes of MIP, NIP, TMIP and TNIP particles were determined by scanning electron microscopy (SEM). The SEM images in Figure 3.8 show both the size and the size distribution of polymer particles. MIP particles were approximately $169-190 \mathrm{~nm}$ in diameter and NIP particles were approximately 112-115 nm. Similarly, TMIP particles were $>180 \mathrm{~nm}$ in diameter and TNIP particles were $>137 \mathrm{~nm}$. These particle sizes were confirmed by dynamic light scattering (DLS) analysis shown in Figure 3.9 that reported a 
diameter of $208 \pm 2 \mathrm{~nm}$ for MIP, $157 \pm 1 \mathrm{~nm}$ for NIP, $212 \pm 2 \mathrm{~nm}$ for TMIP, and $208 \pm 1$ $\mathrm{nm}$ for TNIP. Such similar sizes of the TMIP and TNIP particles are consistent with their electrophoretic mobility values presented in Table 3.8 and Table 3.9

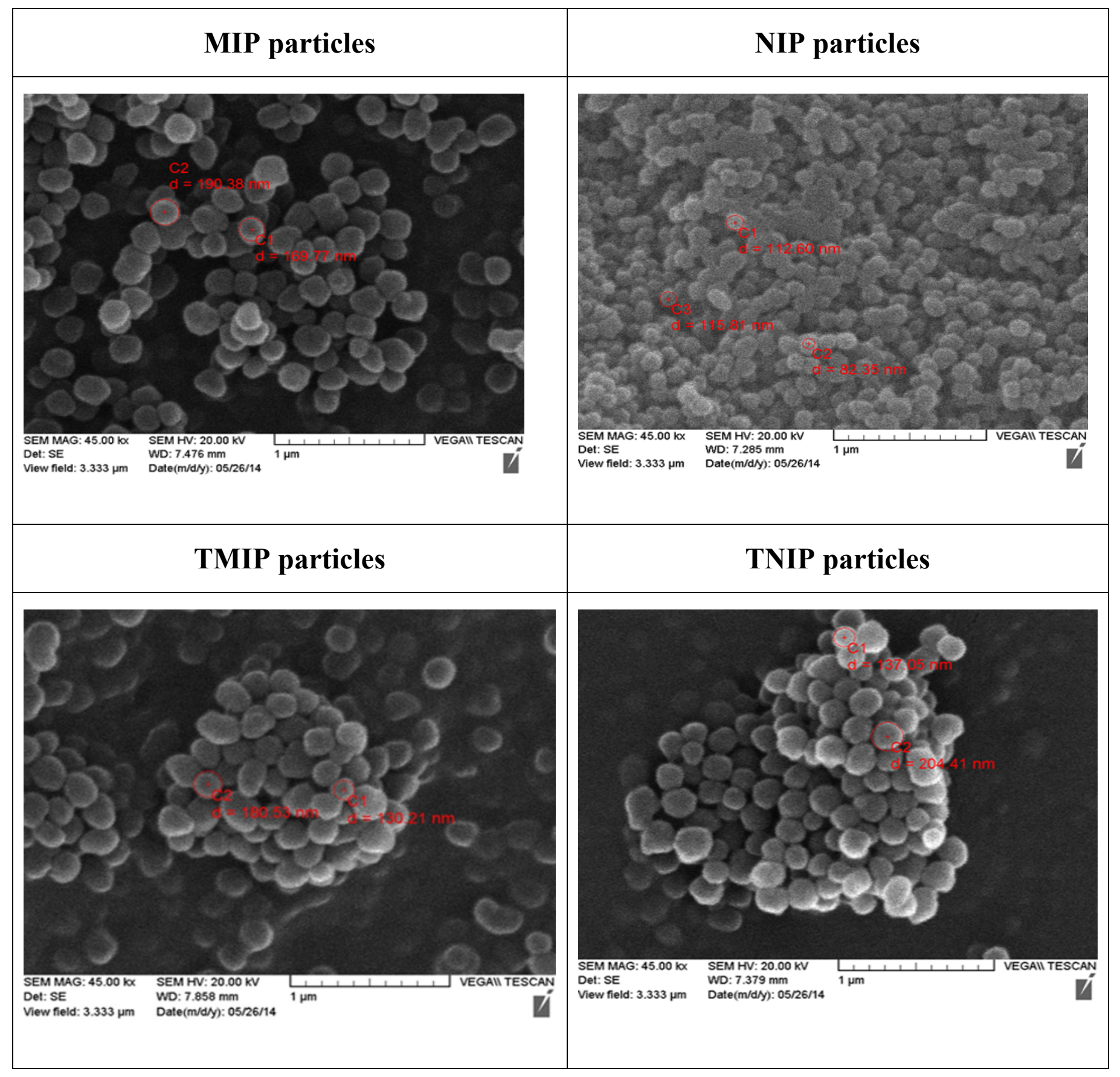

Figure 3.8 SEM analysis of MIP, NIP, TMIP and TNIP particles. 


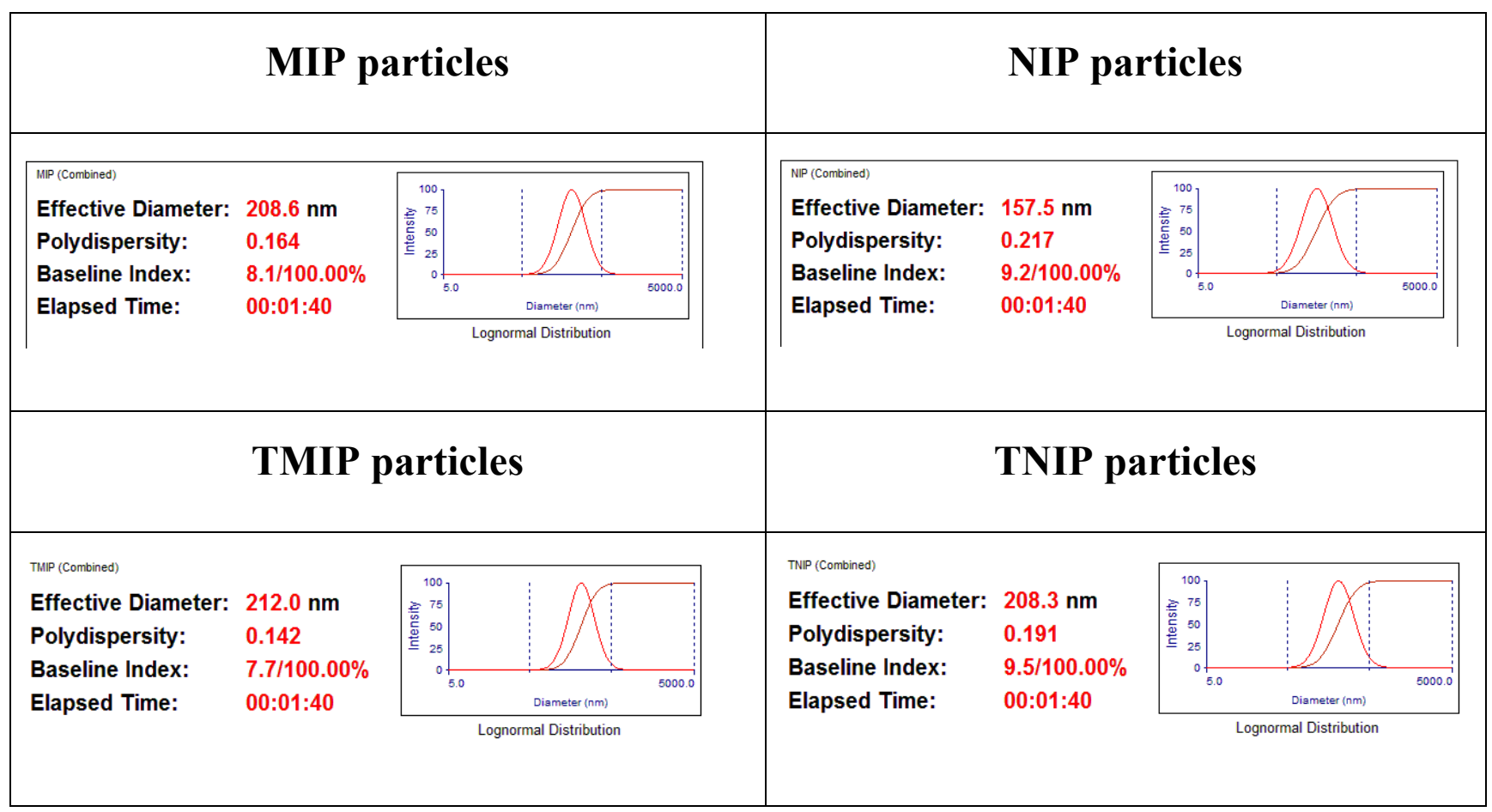

Figure 3.9 DLS analysis of MIP, NIP, TMIP and TNIP particles.

\subsection{Selective Extraction of BPA by TMIP $_{6}$ in Aqueous and and Milk Samples}

\subsubsection{Selective Extraction of BPA in Aqueous Solutions}

For improvement of binding selectivity, our MIPs were subjected to treatment with an ethereal solution of diazomethane, which is known as a powerful methylating agent to esterify carboxylic acid groups [Sammakia, 2001] in the non-specific binding sites. Among all of our TMIPs, TMIP $_{6}$ was chosen to perform selective extraction of BPA in the presence of other compounds that were zwitterionic, negatively, or positively charged in aqueous solutions, and its selectivity was tested by CE-UV and HPLC-UV analyses. $\mathrm{TMIP}_{6}$ results were then compared with a commercially available MIP for BPA, following the same testing condition. The goal of this comparison is to test the efficiency and selectivity of the $\mathrm{TMIP}_{6}$ particles with a well-known commercially available MIP for 
BPA. The chemical structures and $\mathrm{pKa}$ values of those pharmaceutical compounds are presented in Table 3.2 and Table 3.12 .

Table 3.12 Chemical structures and $\mathrm{pK}_{\mathrm{a}}$ values of BPA, BFN, CAF, DFC, DYE, FRU and MF.

\begin{tabular}{|c|c|c|}
\hline Compound & Chemical structure & $\begin{array}{c}\mathbf{p K}_{\mathbf{a}}\left(\mathbf{p K} \mathbf{K}_{\mathbf{b}}\right) \\
\text { Electronic charge state in } 10 \mathrm{mM} \mathrm{Na}_{2} \mathrm{HPO}_{4} \\
(\mathrm{pH} 7.5 \pm 0.2)\end{array}$ \\
\hline $\begin{array}{l}\text { Baclofen } \\
(\mathrm{BFN})\end{array}$ & & $\begin{array}{l}9.5 \text { (3.7) [Castro et al., 1991] } \\
\text { Zwitterionic }\end{array}$ \\
\hline $\begin{array}{l}\text { Caffeine } \\
(\mathrm{CAF})\end{array}$ & & $\begin{array}{c}\text { (0.61) [Hodgman, 1951] } \\
\text { Neutral }\end{array}$ \\
\hline $\begin{array}{l}\text { Dyphylline } \\
\text { (DYE) }\end{array}$ & & $\begin{array}{c}11 \text { [Regan } \text { et al., 2005] } \\
(0.31) \text { [ACD Labs, 2007] } \\
\text { Neutral }\end{array}$ \\
\hline D-Fructose (FRU) & | & $\begin{array}{c}12 \text { [Tang et al., 2007] } \\
\text { Neutral }\end{array}$ \\
\hline
\end{tabular}


As summarized in Table 3.13, when dispersed as $20 \mathrm{mg}$ of particles in $2 \mathrm{~mL}$ of sample, the $\mathrm{TMIP}_{6}$ exhibits highly efficient binding (up to $99 \%$ ) with the target BPA in DDW and BGE. It also demonstrates strong selectivity with significantly lower percent binding with other molecules: 1-8 \% for BFN (zwitterionic), $4-15 \%$ for DFC (negatively charged) and $22-26 \%$ for MF (positively charged). This binding behavior shows both the specific imprinting effect and the non-specific hydrophobic effect. Interestingly, the $\mathrm{TMIP}_{6}$ did not bind with BPA, BFN or DFC in the presence of $\mathrm{MeOH}$ and ACN. The solubility of BPA is substantially higher in both $\mathrm{MeOH}$ and $\mathrm{ACN}$ than in water and this has a highly adverse impact on its binding affinity to the $\mathrm{TMIP}_{6}$. Indeed, $\mathrm{MeOH}$ was used to wash the BPA out of the MIPs and TMIPs. It can be argued that changing the solvent also changed the swelling of the polymer, thus altering the shape of binding cavities. The TMIP 6 was next compared to the commercial MIP. Dispersion of $20 \mathrm{mg}$ of commercial MIP particles in $2 \mathrm{~mL}$ of sample resulted in high binding affinity not only for the BPA target but also toward BFN (34-70\%) and DFC (99\%) in DDW and BGE. This observation was unexpected, in that the binding properties did not seem to be very specific. However, the commercial MIP showed lower binding affinity toward MF than the TMIP 6 . When $2 \mathrm{~mL}$ of a sample containing BPA, BFN, DFC and MF was percolated through the commercial MIP cartridge, BFN (40-93\%) and DFC (96-99\%) were bound in DDW, BGE and DDW/MeOH/ACN. This was another sign that the commercial MIP lacked specific recognition for BPA, particularly in the presence of the zwitterionic and negatively charged molecules, BFN and DFC. All evidence points to the anion exchanger nature of the commercial MIP, which can be compared to the use of 1-allyl-2-thiourea as a functional monomer [Puzio et al., 2014]. 
Table 3.13 Percent binding of commercial MIP cartridge, commercial MIP particles and TMIP $_{6}$ particles with BPA, BFN, DFC and MF spiked in DDW, $10 \mathrm{mM} \mathrm{Na}_{2} \mathrm{HPO}_{4}$ in DDW and DDW/MeOH/ $\mathrm{CH}_{3} \mathrm{CN}(1: 1: 1)$ based on CE-UV and HPLC-UV analyses.

\begin{tabular}{|c|c|c|c|c|c|}
\hline \multicolumn{6}{|c|}{ Percent bindings of target and interfering compounds } \\
\hline \multirow{2}{*}{$\begin{array}{c}\begin{array}{c}\text { Solvent for } \\
\text { sample }\end{array} \\
\text { preparation } \\
\begin{array}{c}\text { Analytical } \\
\text { method }\end{array}\end{array}$} & \multicolumn{2}{|c|}{ DDW } & \multicolumn{2}{|c|}{$\begin{array}{c}\text { BGE } \\
\left(10 \mathrm{mM} \mathrm{Na}_{2} \mathrm{HPO}_{4} \text { in DDW) }\right.\end{array}$} & \multirow{2}{*}{$\begin{array}{c}\text { DDW / } \\
\text { MeOH / ACN } \\
\begin{array}{c}\text { (1:1:1 by } \\
\text { volume })\end{array} \\
\text { HPLC-UV }\end{array}$} \\
\hline & CE-UV & HPLC-UV & CE-UV & HPLC-UV & \\
\hline \multirow{4}{*}{$\underset{\text { particles }^{\text {a }}}{\text { TMIP }_{6}}$} & $\mathrm{BPA}=99 \pm 1$ & $\mathrm{BPA}=80 \pm 1$ & $\mathrm{BPA}=99 \pm 1$ & $\mathrm{BPA}=94 \pm 1$ & $\mathrm{BPA}=0$ \\
\hline & $\mathrm{BFN}=8 \pm 2$ & $\mathrm{BFN}=2 \pm 1$ & $\mathrm{BFN}=7 \pm 3$ & $\mathrm{BFN}=1 \pm 1$ & $\mathrm{BFN}=0$ \\
\hline & $\mathrm{DFC}=5 \pm 1$ & $\mathrm{DFC}=9 \pm 1$ & $\mathrm{DFC}=15 \pm 1$ & $\mathrm{DFC}=4 \pm 1$ & $\mathrm{DFC}=0$ \\
\hline & $\mathrm{MF}=26 \pm 1$ & --- & $\mathrm{MF}=22 \pm 1$ & --- & --- \\
\hline \multirow{4}{*}{$\begin{array}{l}\text { Commercial } \\
\text { MIP }^{\text {particles }}\end{array}$} & $\mathrm{BPA}=99 \pm 1$ & $\mathrm{BPA}=91 \pm 1$ & $\mathrm{BPA}=97 \pm 2$ & $\mathrm{BPA}=83 \pm 2$ & $\mathrm{BPA}=91 \pm 1$ \\
\hline & $\mathrm{BFN}=62 \pm 3$ & $\mathrm{BFN}=56 \pm 1$ & $\mathrm{BFN}=68 \pm 1$ & $\mathrm{BFN}=70 \pm 1$ & $\mathrm{BFN}=34 \pm 1$ \\
\hline & $\mathrm{DFC}=99 \pm 1$ & $\mathrm{DFC}=99 \pm 1$ & $\mathrm{DFC}=99 \pm 1$ & $\mathrm{DFC}=80 \pm 2$ & $\mathrm{DFC}=99 \pm 1$ \\
\hline & $\mathrm{MF}=4 \pm 1$ & --- & $\mathrm{MF}=5 \pm 2$ & --- & --- \\
\hline \multirow{4}{*}{$\begin{array}{l}\text { Commercial } \\
\text { MIP } \\
\text { cartridge }^{b}\end{array}$} & $\mathrm{BPA}=99 \pm 1$ & $\mathrm{BPA}=97 \pm 1$ & $\mathrm{BPA}=99 \pm 1$ & $\mathrm{BPA}=98 \pm 1$ & $\mathrm{BPA}=85 \pm 1$ \\
\hline & $\mathrm{BFN}=68 \pm 1$ & $\mathrm{BFN}=68 \pm 1$ & $\mathrm{BFN}=93 \pm 1$ & $\mathrm{BFN}=89 \pm 1$ & $\mathrm{BFN}=40 \pm 1$ \\
\hline & $\mathrm{DFC}=99 \pm 1$ & $\mathrm{DFC}=96 \pm 3$ & $\mathrm{DFC}=99 \pm 1$ & $\mathrm{DFC}=99 \pm 1$ & $\mathrm{DFC}=99 \pm 1$ \\
\hline & $\mathrm{MF}=22 \pm 1$ & --- & $\mathrm{MF}=14 \pm 1$ & --- & --- \\
\hline
\end{tabular}

${ }^{a} 20 \mathrm{mg}$ dispersed in $2 \mathrm{~mL}$ of sample. $\quad{ }^{b} 100 \mathrm{mg}$ of particles for $2 \mathrm{~mL}$ of sample. 


\subsubsection{Selective Extraction of BPA in Milk Samples}

Dilution of milk with BGE was a simple and effective means of sample preparation for CE-UV analysis. Spiking the sample with BPA, BFN, CAF, DFC, DYE, FRU and MF enabled comparison of the binding properties of the $\mathrm{TMIP}_{6}$ and the commercial MIP toward BPA in milk. CE allowed the centrifugation step to be omitted and the particles would just appear as a broad peak (extending from 5 min to $16 \mathrm{~min}$ ) above the electropherogram baseline, as shown for the commercial MIP particles with DYE in Figure 3.10 (b). As presented in Table 3.14, both the $\mathrm{TMIP}_{6}$ and commercial MIP (whether as dispersed particles or a packed cartridge) recovered BPA in the spiked milk samples at the $80-85 \%$ binding level. They both demonstrated low bindings toward positively charged compound MF (16-20\% at $200 \mathrm{ppm}$ ). CE-UV analysis of lactose (which makes up $2-8 \%$ of milk by weight) was not feasible because lactose does not absorb much $190 \mathrm{~nm}$ UV light, even at a concentration of $30 \mathrm{mg} / \mathrm{mL}$; however, FRU exhibited a low binding affinity for both polymers $(8-10 \%$ at $30000 \mathrm{ppm}$, partly due to saturation of available non-specific sites). However, the commercial MIP exhibited high affinity toward the nitrogenous neutral compounds CAF (39-51\%) and DYE (16-63\%), as well as the zwitterionic BFN (50-78\%), indicating a lack of specificity for the BPA target. This is in stark contrast to the $\mathrm{TMIP}_{6}$, which weakly bound to BFN (3\%), CAF (11\%) and DYE (6\%). Apparently the $\mathrm{TMIP}_{6}$ was highly selective toward BPA in the presence of these neutral compounds, unlike the commercial MIP. 

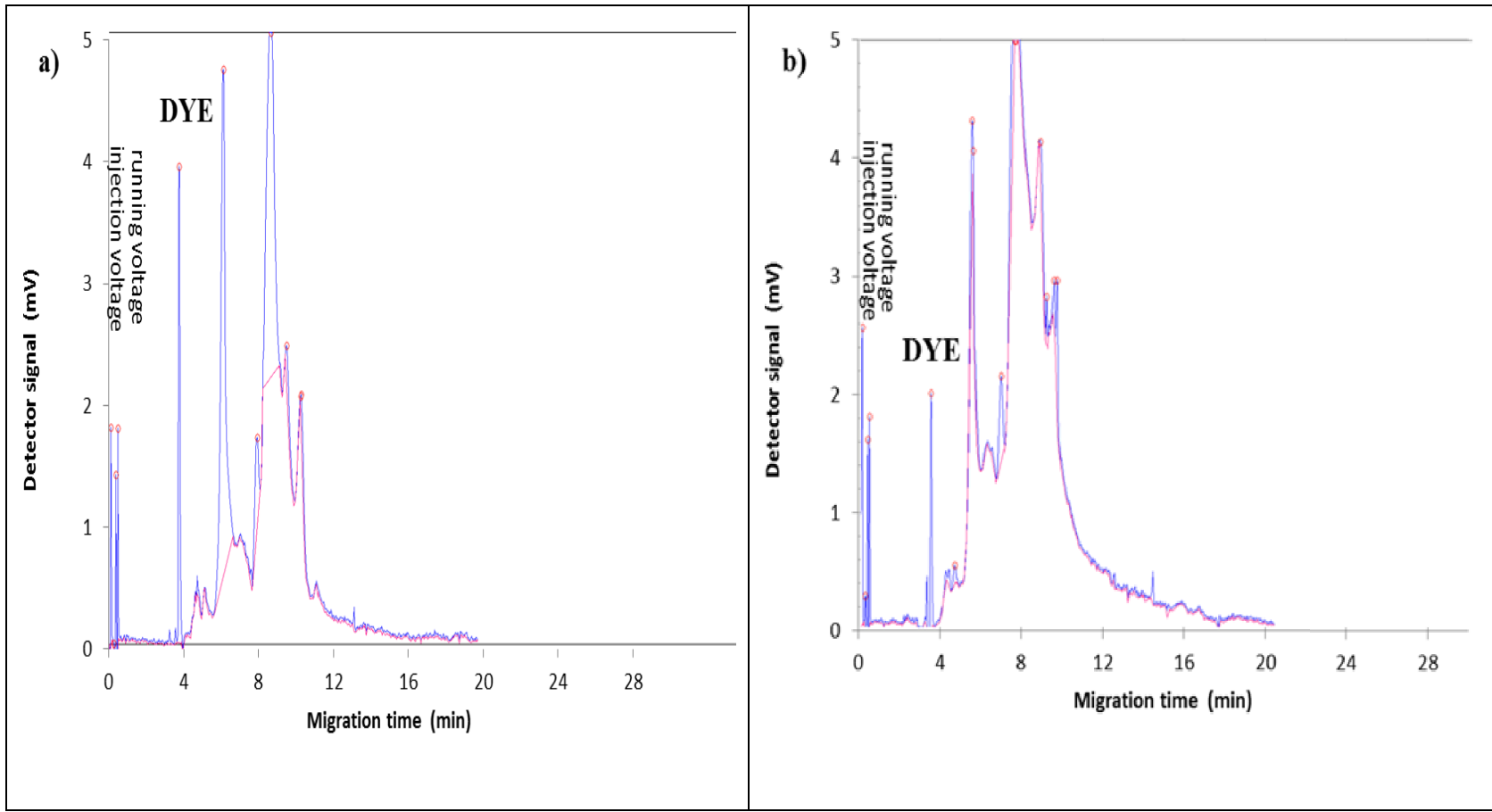

Figure 3.10 Electropherograms of milk spiked with DYE (a) before binding with commercial MIP particles, and (b) after binding with commercial MIP particles with no centrifugation. 
Table 3.14 Percent bindings of TMIP particles, commercial MIP particles and commercial MIP cartridge with BPA, BFN, CAF, DFC, DYE, FRU and MF spiked in milk samples, based on CE-UV analyses.

\begin{tabular}{|c|c|}
\hline $\begin{array}{l}\text { Solid phase extraction after spiking milk with } \\
\text { standard solution (to final concentration) }\end{array}$ & $\begin{array}{l}\text { Percent bindings of target and } \\
\text { interfering compounds }\end{array}$ \\
\hline TMIP $_{6}$ particles $^{\text {a }}$ & $\mathrm{BPA}=85 \pm 1$ \\
\hline BPA (200 ppm) and MF (200 ppm) & $\mathrm{MF}=18 \pm 1$ \\
\hline BFN (200 ppm) & $\mathrm{BFN}=3 \pm 2$ \\
\hline CAF (200 ppm) & $\mathrm{CAF}=11 \pm 1$ \\
\hline DYE (200 ppm) & $\mathrm{DYE}=6 \pm 1$ \\
\hline FRU (30000 ppm) & $\mathrm{FRU}=8 \pm 2$ \\
\hline Commercial MIP particles ${ }^{\text {a }}$ & $\mathrm{BPA}=82 \pm 1$ \\
\hline BPA (200 ppm) and MF (200 ppm) & $\mathrm{MF}=20 \pm 1$ \\
\hline BFN (200 ppm) & $\mathrm{BFN}=50 \pm 1$ \\
\hline CAF (200 ppm) & $\mathrm{CAF}=39 \pm 2$ \\
\hline DYE (200 ppm) & $\mathrm{DYE}=50 \pm 4$ \\
\hline FRU (30000 ppm) & $\mathrm{FRU}=10 \pm 2$ \\
\hline Commercial MIP cartridge ${ }^{b}$ & $\mathrm{BPA}=82 \pm 2$ \\
\hline BPA (100 ppm) and MF (200 ppm) & $\mathrm{MF}=16 \pm 2$ \\
\hline BFN (200 ppm) & $\mathrm{BFN}=78 \pm 2$ \\
\hline CAF(200 ppm) & $\mathrm{CAF}=51 \pm 2$ \\
\hline DYE (200 ppm) & $\mathrm{DYE}=63 \pm 1$ \\
\hline FRU (30000 ppm) & $\mathrm{FRU}=8 \pm 2$ \\
\hline
\end{tabular}

${ }^{a} 20 \mathrm{mg}$ dispersed in $2 \mathrm{~mL}$ of sample. $\quad{ }^{b} 100 \mathrm{mg}$ of particles for $2 \mathrm{~mL}$ of sample. 


\section{Chapter 4}

Conclusions

and

Future Work 


\section{Conclusions and Future Work}

\subsection{Conclusions}

Treating the BPA-molecularly imprinted polymer particles with diazomethane to esterify the undesired non-specific binding sites (and maintain the desired specific binding sites intact) has been achieved. In the first stage of this project, selective post chemical modification of MIPs with diazomethane was successfully done. When the selective treatment was completed, and the template was then removed, the selectivity of TMIP 1 toward BPA, in the presence of other organic compounds, compared favorably with nontreated $\mathrm{MIP}_{1}$. Even in the presence of positively charged compounds and negatively charged compounds, the treated imprinted particles afford higher selectivity for BPA in comparison with non-esterified imprinted particles. A very significant decrease in affinity of metformin (a positively charged compound) toward the $\mathrm{TMIP}_{1}$ particles was also observed in comparison with the non-esterified MIP particles. To prove the partial esterification of TMIP ${ }_{1}$ by diazomethane, electrophoretic mobility and solid-state ${ }^{13} \mathrm{C}$ NMR methods were used to measure the negative charge and to examine the carbonyl signals of $\mathrm{TMIP}_{1}$, respectively, in comparison with non-treated $\mathrm{MIP}_{1}$. Electrophoretic mobility results show that $\mathrm{TMIP}_{1}$ has a lower negative charge than the non-treated $\mathrm{MIP}_{1}$, which is an indication of a decrease in the number of carboxylic acids (methyl methacrylic acids) as a result of the partial conversion to MMA (methyl methacrylate) by diazomethane. The carbonyl peak of $\mathrm{TMIP}_{1}$ in the solid-state ${ }^{13} \mathrm{C}$ NMR spectrum is also further evidence for the partial esterification of MAA monomers to the corresponding MMA. The TMIP ${ }_{1}$ carbonyl peak is shifted to a more shielded position (177.6) ppm in comparison with $\mathrm{MIP}_{1}$, which has a carbonyl peak at $178.1 \mathrm{ppm}$. This trend of shifting of 
the TMIP ${ }_{1}$ carbonyl peak is a well-known phenomenon for the conversion of an acid to its corresponding methyl ester.

In the second stage of this project, another approach - the optimization of template to functional monomer ratio - was explored in parallel to, and in conjunction with, SSCM. This approach was based on increasing the molar ratio of BPA (the template molecule) to MAA (the functional monomer). The results of this approach show that as the ratio of BPA to MAA increases from 1:9 (in $\mathrm{MIP}_{1}$ ) to 9:1 (in $\mathrm{MIP}_{10}$ ), the binding selectivity toward the target analyte (BPA) is enhanced. Specifically, $\mathrm{MIP}_{10}$ has the lowest binding affinity toward metformin in comparison with other MIPs, which have lower BPA: MAA molar ratios. However, although the binding affinity toward the positively charged compound (MF) is improved (from $81 \%$ for $\mathrm{MIP}_{2}$ to $33 \%$ for $\mathrm{MIP}_{10}$ ), the binding affinity toward the negatively charged compound (DFC) is slightly increased (from $11 \%$ for $\mathrm{MIP}_{2}$ to $25 \%$ for $\mathrm{MIP}_{10}$ ); the $\mathrm{DFC}$ increase was mainly due to elevated hydrophobic interactions from $\mathrm{MIP}_{2}$ to $\mathrm{MIP}_{10}$. To enhance the selectivity of all these modified MIPs, the diazomethane approach was combined with this ratio approach. The results show that as the ratio of BPA to MAA increases (starting from $\mathrm{TMIP}_{5}$ to $\mathrm{TMIP}_{10}$ ), the binding affinity toward BPA increase probably due to an increase in the number of specific binding sites. All the treated MIPs $\left(\mathrm{TMIP}_{2}\right.$ to $\left.\mathrm{TMIP}_{10}\right)$ show a lower binding affinity toward the negatively charged compound (DFC), the positively charged compound (MF), and the zwitterionic compound (BFN) in comparison with non-treated MIPs $\left(\mathrm{MIP}_{2}\right.$ to $\mathrm{MIP}_{10}$ ). In summary, the diazomethane treatment approach is further proven to be a powerful technique to eliminate the undesired sites in MIPs, and using the optimization of template to functional monomer ratio is an excellent approach to increase the specific 
binding sites in MIPs. Hence, by combining those two approaches, selective TMIPs $\left(\mathrm{TMIP}_{5}\right.$ to $\left.\mathrm{TMIP}_{10}\right)$ toward $\mathrm{BPA}$, in the presence of other charged molecules, were successfully obtained.

In the third stage of this M.Sc. project, $\mathrm{TMIP}_{6}$ was chosen to perform selective extraction of BPA in both aqueous solutions and milk samples and its binding results were compared with those for a commercially available MIP. Both HPLC-UV and CE-UV analysis results show that $\mathrm{TMIP}_{6}$ exhibits highly efficient binding with the target BPA (up to 99\%), and it has a lower binding affinity toward the negatively charged compound (DFC) in comparison with the commercial MIP in both DDW and BGE. In milk analysis, $\mathrm{CE}$ proved itself to be an effective technique for the separation of BPA from all constituents of milk. The results indicated $\mathrm{TMIP}_{6}$ can selectively extracted BPA in the presence of zwitterionic (DFC), positively charged molecules (MF) and neutral molecules (CAF, DYE, and FRU) in milk samples. Contrary to $\mathrm{TMIP}_{6}$, the commercial MIP did not show high selectivity for BPA extraction in the presence of zwitterionic BFN, anionic DFC, or neutral CAF and DYE compounds. One plausible explanation is that the commercial MIP has an anion exchanger nature. 


\subsection{Future Work}

MIP-based sensors for the determination of toxic molecules in contaminated environments have recently attracted analytical chemists. The fundamental element in this approach is to create a connection between the target binding event and a signal transducer. When MIP particles are combined with a transducer in a proper formation, sensors along with the recognition elements can identify and quantify the target compound by converting the target compound-MIP rebinding into a quantifiable signal as shown in Figure 4.1.

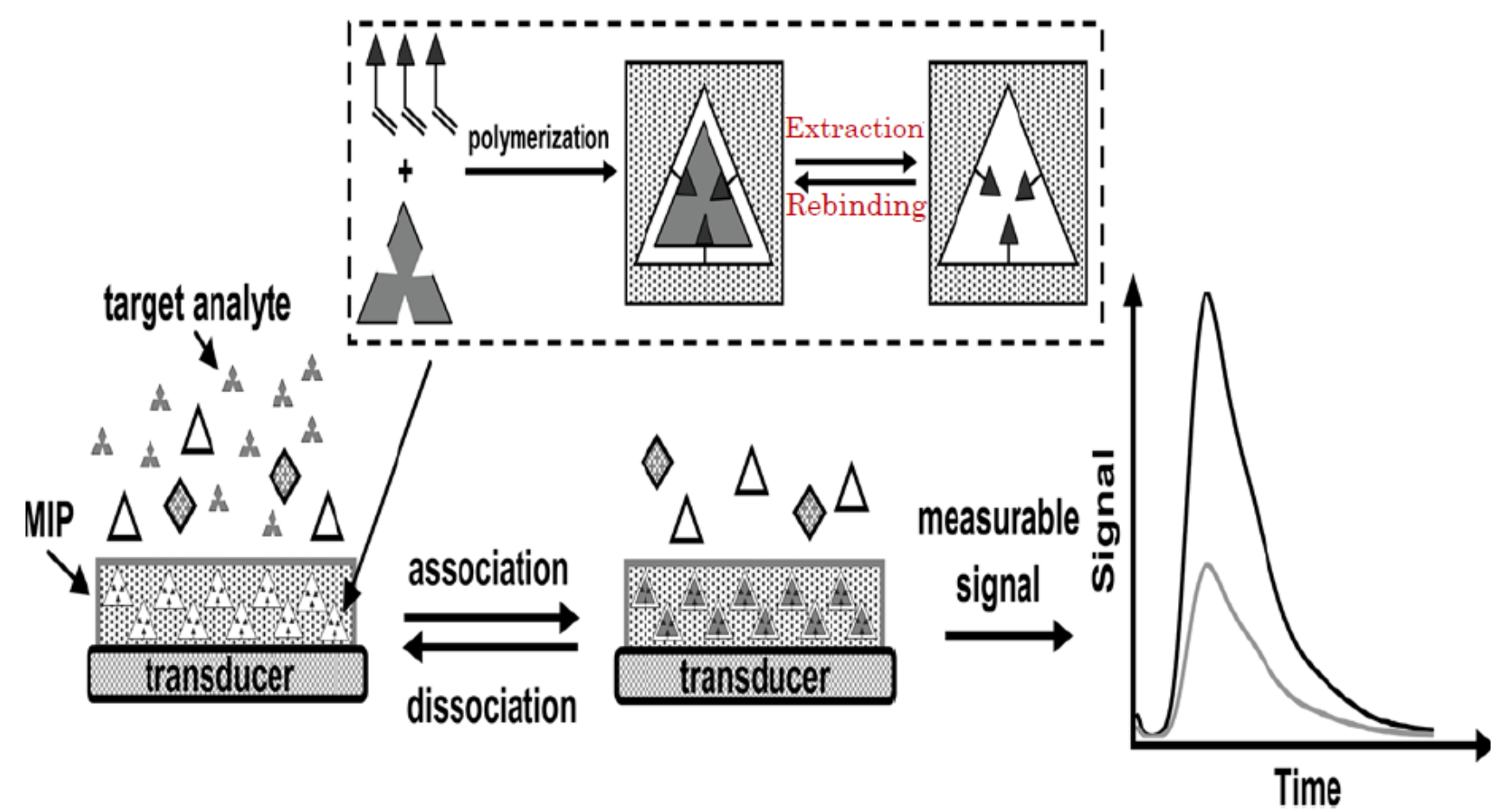

Figure 4.1 MIP-based sensors (Guan et al., 2008).

In this project, after our examination of the selectivity of TMIP toward its target analyte (BPA), our TMIP particles could be a useful material in sensor applications such as a BPA sensor. Thus, TMIP based sensors will be made by the assembly of TMIP particles 
onto the transducer surface, so when the target analyte (BPA) rebinds into the recognition element in the TMIP, the binding creates to a measurable signal. It is hoped that we can establish fast and low-cost TMIP-based BPA sensors; however, the transducer mechanism and engineering design are still currently challenging to many experts in the sensor field and this research will not be trivial. 


\section{References}

ACD Labs (2007). Advanced Chemistry Development Software V11.02.

Alsudir, S., Iqbal, Z., \& Lai, E. P. C., (2012). Competitive CE-UV binding tests for selective recognition of bisphenol A by molecularly imprinted polymer particles. Electrophoresis, 33(8), 1255-1262.

Alsudir, S., \& Lai, E. P. C., (2013). High Specificity of Molecularly Imprinted Polymer Particles toward Target Compound in Competitive Environmental Binding. European Chemical Bulletin, 2(3), 112-118.

Angiolini, L., Caretti, D., Salatelli, E., Mazzocchetti, L., Willem, R., \& Biesemans, M. (2008). Synthesis and characterization of new functional polystyrenes containing tributyltin carboxylate moieties linked to the aromatic ring by a trimethylene spacer. Journal of Inorganic and Organometallic Polymers and Materials, 18(2), 236-245.

Anisimov, V., (2014). Do metformin a real anticarcinogen? A critical reappraisal of experimental data. Annals of Translational Medicine; Vol 2, No 6.

Bansal, V. (2010). High performance liquid chromatography: a short review. Journal of Global Pharma Technology, 2(5).

Bao, B., Azmi, A. S., Ali, S., Zaiem, F., \& Sarkar, F. H. (2014). Metformin may function as anti-cancer agent via targeting cancer stem cells: the potential biological significance of tumor-associated miRNAs in breast and pancreatic cancers. Annals of translational medicine, 2(6). 
Bing-zhi, D., Hua-qiang, C., Lin, W., Sheng-ji, X., \& Nai-yun, G. (2010). The removal of bisphenol A by hollow fiber microfiltration membrane. Desalination, 250(2), 693-697.

Bosserhoff, K., \& Hellerbrand, C., (2005). Capillary electrophoresis. Molecular Diagnostics. Patrinos, G., \& Ansorge, W., (Eds). Elsevier; pp. 67-69.

Breitmaier, E., \& Voelter, W. (1987). Carbon-13 NMR Spectroscopy. High-Resolution Methods and Applications in Organic Chemistry and Biochemistry, 3rd Ed.; VCH: Weinheim; pp. 225-229.

Calafat, A. M., Ye, X., Wong, L. Y., Reidy, J. A., \& Needham, L. L. (2008). Exposure of the US population to Bisphenol A and 4-tertiary-Octylphenol: 2003-2004. Environmental Health Perspectives, 39-44.

Canada Gazette (2013). Order adding a toxic substance to Schedule 1 to the Canadian Environmental Protection Act, 1999. Canadian Environmental Protection Act, 1999.Vol. 144 (21). http://www.gazette.gc.ca/rp-pr/p2/2010/2010-10-13/html/sor-dors194-eng.html (accessed Oct 6, 2014).

Castro, J., Meynadier, J., \& Zenz, M. (1991). Regional opioid analgesia:

Physiopharmacological basis, drugs, equipment, and clinical application. Dordrecht: Kluwer Academic Publishers. p.181.

Chen, L., Xu, S., \& Li, J. (2011). Recent advances in molecular imprinting technology: current status, challenges and highlighted applications. Chemical Society Reviews, 40(5), 2922-2942. 
Cheong, W. J., Yang, S. H., \& Ali, F. (2013). Molecular imprinted polymers for separation science: A review of reviews. Journal of Separation Science, 36(3), 609-628.

Cho, S., Choi, Y. S., Luu, H. M. D., \& Guo, J. (2012). Determination of total leachable bisphenol A from polysulfone membranes based on multiple consecutive extractions. Talanta, 101, 537-540.

Clara, M., Strenn, B., Saracevic, E., \& Kreuzinger, N. (2004). Adsorption of bisphenolA, 17ß-estradiole and 17 $\alpha$-ethinylestradiole to sewage sludge. Chemosphere, 56(9), 843851.

Cormack, P. A., \& Elorza, A. Z. (2004). Molecularly imprinted polymers: synthesis and characterisation. Journal of chromatography B, 804(1), 173-182.

Courtois, J., Fischer, G., Sellergren, B., \& Irgum, K. (2006). Molecularly imprinted polymers grafted to flow through poly (trimethylolpropane trimethacrylate) monoliths for capillary-based solid-phase extraction. Journal of Chromatography A, 1109(1), 92-99.

Coulter, B. (1991). Introduction to capillary electrophoresis. Beckman Coulter. https://www.beckmancoulter.com/wsrportal/bibliography?docname $=360643$ CEPrimerl.pdf. (accessed on October 06, 2014).

Cousins, I. T., Staples, C. A., Kleĉka, G. M., \& Mackay, D. (2002). A multimedia assessment of the environmental fate of bisphenol A. Human and Ecological Risk Assessment, 8(5), 1107-1135. 
Daughton, C. G. (2004). Non-regulated water contaminants: emerging research. Environmental Impact Assessment Review, 24(7), 711-732.

De Boer TJ, Backer HJ. (1954). p-Tolylsulfonylmethylnitrosamide. Organic Syntheses, 34: $96-99$.

Del Olmo, M., Gonzalez-Casado, A., Navas, N. A., \& Vilchez, J. L. (1997). Determination of bisphenol A (BPA) in water by gas chromatography-mass spectrometry. Analytica Chimica Acta, 346(1), 87-92.

DeMaleki, Z., Lai, E. P. C., \& Dabek-Zlotorzynska, E. (2010). Capillary Electrophoresis Characterization of Molecularly Imprinted Polymer Particles in Fast Binding with 17 $\beta$ Estradiol. J. Sep. Sci. 33 (17-18): 2796-2803. DOI: 10.1002/jssc. 201000257.

Dong, M. W. (2006). Modern HPLC for practicing scientists. John Wiley \& Sons. http://samples.sainsburysebooks.co.uk/9780471973096_sample_388122.pdf. (accessed on October 06, 2014).

Drug bank (2005). Baclofen. http://www.drugbank.ca/drugs/DB00181 (accessed on June 22, 2014).

Duncan, S. D., \& Devlin, L. A. (2013). Use of baclofen for withdrawal in a preterm infant. Journal of Perinatology, 33(4), 327-328.

Gao, Q., Luo, D., Bai, M., Chen, Z. W., \& Feng, Y. Q. (2011). Rapid determination of estrogens in milk samples based on magnetite nanoparticles/polypyrrole magnetic solidphase extraction coupled with liquid chromatography-tandem mass spectrometry. Journal of Agricultural and Food Chemistry, 59(16), 8543-8549. 
Glynn, J. R., Belongia, B. M., Arnold, R. G., Ogden, K. L., \& Baygents, J. C. (1998). Capillary electrophoresis measurements of electrophoretic mobility for colloidal particles of biological interest. Applied and Environmental Microbiology, 64(7), 2572-2577.

Greene, N. T., \& Shimizu, K. D. (2005). Colorimetric molecularly imprinted polymer sensor array using dye displacement. Journal of American Chemical Society, 127(15), 5695-5700.

Guan, G., Liu, B., Wang, Z., \& Zhang, Z. (2008). Imprinting of molecular recognition sites on nanostructures and its applications in chemosensors. Sensors, 8(12), 8291-8320.

Haupt, K., \& Mosbach, K. (2000). Molecularly imprinted polymers and their use in biomimetic sensors. Chemical Reviews, 100(7), 2495-2504.

Haupt, K. (2001). Molecularly imprinted polymers in analytical chemistry. Analyst, $126(6), 747-756$.

Hodgman, C. D. (Ed.) (1951). Handbook of Chemistry and Physics. CRC Press: Cleveland, pp. 1636-7.

Hu, Y., Pan, J., Zhang, K., Lian, H., \& Li, G. (2013). Novel applications of molecularlyimprinted polymers in sample preparation. TrAC Trends in Analytical Chemistry, 43, 3752.

Huang, W. S., Lin, S. J., Wu, H. L., \& Chen, S. H. (2003). Simultaneous determination of theophylline and dyphylline by micellar electrokinetic chromatography and application in drug formulations. Journal of Chromatography B, 795(2), 329-335. 
Inoue, K., Kato, K., Yoshimura, Y., Makino, T., \& Nakazawa, H. (2000). Determination of bisphenol A in human serum by high-performance liquid chromatography with multielectrode electrochemical detection. Journal of Chromatography B: Biomedical Sciences and Applications, 749(1), 17-23.

Ji, Y., Yin, J., Xu, Z., Zhao, C., Huang, H., Zhang, H., \& Wang, C. (2009). Preparation of magnetic molecularly imprinted polymer for rapid determination of bisphenol $\mathrm{A}$ in environmental water and milk samples. Analytical and Bioanalytical Chemistry, 395(4), 1125-1133.

Karim, K., Breton, F., Rouillon, R., Piletska, E. V., Guerreiro, A., Chianella, I., \& Piletsky, S. A. (2005). How to find effective functional monomers for effective molecularly imprinted polymers? Advanced drug delivery reviews, 57(12), 1795-1808.

Kashiwada, S., Ishikawa, H., Miyamoto, N., Ohnishi, Y., \& Magara, Y. (2002). Fish test for endocrine-disruption and estimation of water quality of Japanese rivers. Water Research, 36(8), 2161-2166.

Kawahata, H., Ohta, H., Inoue, M., \& Suzuki, A. (2004). Endocrine disrupter nonylphenol and bisphenol A contamination in Okinawa and Ishigaki Islands, Japanwithin coral reefs and adjacent river mouths. Chemosphere, 55(11), 1519-1527.

Kester, M. B., Saccar, C. L., \& Mansmann Jr, H. C. (1987). Microassay for the simultaneous determination of theophylline and dyphylline in serum by highperformance liquid chromatography. Journal of Chromatography B: Biomedical Sciences and Applications, 416, 91-97. 
Koenhen, D. M., \& Smolders, C. A. (1975). The determination of solubility parameters of solvents and polymers by means of correlations with other physical quantities. Journal of Applied Polymer Science, 19(4), 1163-1179.

Krozer, A., Löfving, B., Malm, B., Reimhult, K., Ye, L., \& Yoshimatsu, K. (2010). Customized molecularly imprinted polymer (mip) units. U.S. Patent Application $13 / 147,631$.

Lai, E. P. C., \& Feng, S. Y. (2006). Solid phase extraction-Non-aqueous capillary electrophoresis for determination of metformin, phenformin and glyburide in human plasma. Journal of Chromatography B, 843(1), 94-99.

Lai, E. P. C., Dzhun, A., \& De Maleki, Z. (2010). Molecularly imprinted polymer submicron particles tailored for extraction of trace estrogens in water. In Trace Analysis with Nanomaterials. Pierce, D., \& Zho, J., (Eds). (1st ed., pp. 135-155). Wiley-VCH.

Lau, O. W., \& Wong, S. K. (2000). Contamination in food from packaging material. Journal of Chromatography A, 882(1), 255-270.

Dolson, L. (2006). Fructose: Sweet, But Dangerous. Diabetes Care, 29, 2140-2157.

Martín-Esteban, A. (2013). Molecularly-imprinted polymers as a versatile, highly selective tool in sample preparation. TrAC Trends in Analytical Chemistry, 45, 169-181. 
Masque, N., Marce, R. M., \& Borrull, F. (2001). Molecularly imprinted polymers: new tailor-made materials for selective solid-phase extraction. TrAC Trends in Analytical Chemistry, 20(9), 477-486.

McNiven, S., Yokobayashi, Y., Cheong, S. H., \& Karube, I. (1997). Enhancing the selectivity of molecularly imprinted polymers. Chemistry Letters, (12), 1297-1298.

Mei, S., Wu, D., Jiang, M., Lu, B., Lim, J. M., Zhou, Y. K., \& Lee, Y. I. (2011). Determination of trace bisphenol A in complex samples using selective molecularly imprinted solid-phase extraction coupled with capillary electrophoresis. Microchemical Journal, 98(1), 150-155.

Metrohm USA Ins. (2010). Cumene process: Analysis of Sulfuric Acid in Acetone and Phenol. Note ON-PAN-1008-cumene-to-phenol-process. http://www.metrohm-applikon.com/Downloads/Process_Application_Note_AN-PAN1008-cumene-to-phenol-process. (accessed on October 06, 2014).

Mullett, W. M., \& Lai, E. P. C., (1999). Rapid determination of theophylline in serum by selective extraction using a heated molecularly imprinted polymer micro-column with differential pulsed elution. Journal of Pharmaceutical and Biomedical analysis, 21(4), $835-843$.

Nicholls, I. A., Ramström, O., \& Mosbach, K. (1995). Insights into the role of the hydrogen bond and hydrophobic effect on recognition in molecularly imprinted polymer synthetic peptide receptor mimics. Journal of Chromatography A, 691(1), 349-353. 
Öpik, A., Menaker, A., Reut, J., \& Syritski, V. (2009). Molecularly imprinted polymers: a new approach to the preparation of functional materials. Proceedings of the Estonian Academy of Sciences, 58(1), 3-11.

Pardeshi, S., Dhodapkar, R., \& Kumar, A. (2014). Influence of porogens on the specific recognition of molecularly imprinted poly (acrylamide-co-ethylene glycol dimethacrylate). Composite Interfaces, 21(1), 13-30.

Prieto, A., Basauri, O., Rodil, R., Usobiaga, A., Fernández, L. A., Etxebarria, N., \& Zuloaga, O. (2010). Stir-bar sorptive extraction: a view on method optimisation, novel applications, limitations and potential solutions. Journal of Chromatography A, 1217(16), 2642-2666.

Puzio, K., Claude, B., Amalric, L., Berho, C., Grellet, E., Bayoudh, S., ... \& Morin, P. (2014). Molecularly imprinted polymer dedicated to the extraction of glyphosate in natural waters. Journal of Chromatography A, 1361, 1-8.

Quednow, K., \& Püttmann, W. (2008). Endocrine disruptors in freshwater streams of Hesse, Germany: changes in concentration levels in the time span from 2003 to 2005. Environmental Pollution, 152(2), 476-483.

Regan, F., \& Shakalisava, Y. (2005). Rapid simultaneous determination of alkylxanthines by CZE and its application in analysis of pharmaceuticals and food samples. Analytica Chemical Acta, 540(1), 103-110.

Ren, Y. M., Yang, J., Ma, W. Q., Ma, J., Feng, J., \& Liu, X. L. (2014). The selective binding character of a molecular imprinted particle for Bisphenol A from water. Water Research, 50, 90-100. 
Rocha, S., Domingues, V. F., Pinho, C., Fernandes, V. C., Delerue-Matos, C., Gameiro, P., \& Mansilha, C. (2013). Occurrence of Bisphenol A, Estrone, 17 $\beta$-Estradiol and 17 $\alpha$ Ethinylestradiol in Portuguese Rivers. Bulletin of Environmental Contamination and Toxicology, 90(1), 73-78.

Röhricht, M., Krisam, J., Weise, U., Kraus, U. R., \& Düring, R. A. (2009). Elimination of carbamazepine, diclofenac and naproxen from treated wastewater by nanofiltration. CLEAN-Soil, Air, Water, 37(8), 638-641.

Roper, R., \& Ma, T. S. (1957). Diazomethane as a reagent for microsynthesis. Microchemical Journal, 1(2), 245-260.

Sellergren, B., \& Shea, K. J. (1993). Influence of polymer morphology on the ability of imprinted network polymers to resolve enantiomers. Journal of Chromatography A, 635(1), 31-49.

Sellergren, Börje, \& Andrew, J. Hall. (2000). Fundamental aspects on the synthesis and characterisation of imprinted network polymers. Molecularly imprinted polymers: manmade mimics of antibodies and their application in analytical chemistry. Vol. 23: 21-57.

Sandoz Inc. (2007). Diclofenac Sodium (Diclofenac Sodium). http://dailymed.nlm.nih.gov/dailymed/archives/fdaDrugInfo.cfm?archiveid=3007. (accessed June 22, 2014). 
Sammakia, T. (2001). Diazomethane. E-EROS Encyclopedia of Reagents for Organic Synthesis. http://onlinelibrary.wiley.com/doi/10.1002/047084289X.rd017/full (accessed August 11, 2014).

Svec, F., \& Frechet, J. M. J. (1996). Pore-size specific modification as an approach to separation media for single-column, two-dimensional HPLC. American laboratory, $28(18), 25-34$.

Shao, B., Han, H., Tu, X., \& Huang, L. (2007). Analysis of alkylphenol and bisphenol A in eggs and milk by matrix solid phase dispersion extraction and liquid chromatography with tandem mass spectrometry. Journal of Chromatography B, 850(1), 412-416.

Shimizu, K. D. (2005). Post modification of imprinted polymers. In Molecularly Imprinted Materials: Science and Technology, Yan, M., \& Ramström, O. (Eds.). Marcel Dekker: New York. pp. 285-306.

Sigma-Aldrich Technical Bulletin AL-180. (2007). Diazald ${ }^{\circledR}$ and Diazomethane Generators.http://www.sigmaaldrich.com/content/dam/sigmaaldrich/docs/Aldrich/Bulleti n/al_techbull_al180.pdf (accessed on June 13, 2014).

Snyder, L. R. (1978). Classification off the Solvent Properties of Common Liquids. Journal of Chromatographic Science, 16(6), 223-234.

Sun, Y., Irie, M., Kishikawa, N., Wada, M., Kuroda, N., \& Nakashima, K. (2004). Determination of bisphenol A in human breast milk by HPLC with column-switching and fluorescence detection. Biomedical Chromatography, 18(8), 501-507. 
Stierlin, H., Faigle, J. W., Sallmann, A., Kung, W., Richter, W. J., Kriemler, H. P., \& Winkler, T. (1979). Biotransformation of diclofenac sodium (Voltaren $®$ ) in animals and in man: I. Isolation and identification of principal metabolites. Xenobiotica, 9(10), 601610.

Stackelberg, P. E., Gibs, J., Furlong, E. T., Meyer, M. T., Zaugg, S. D., \& Lippincott, R. L. (2007). Efficiency of conventional drinking-water-treatment processes in removal of pharmaceuticals and other organic compounds. Science of the Total Environment, 377(2), 255-272.

Tamayo, F. G., Turiel, E., \& Martín-Esteban, A. (2007). Molecularly imprinted polymers for solid-phase extraction and solid-phase microextraction: Recent developments and future trends. Journal of Chromatography A, 1152(1), 32-40.

Tang, K., Liang, L., Cai, Y., \& Mou, S. (2007). Determination of sugars and alditols in tobacco with high performance anion-exchange chromatography. Journal of separation Science, 30(13), 2160-2166.

The Vancouver Sun. (2014).

http://www.vancouversun.com/health/teen+died+after+overdosing+caffeine/10045479/st ory.html (accessed on July 28, 2014).

Tosoh Bioscience LLC. (2009). The Chemistry of Innovation. Principles of Reversed Phase Chromatography. http://www.separations.us.tosohbioscience.com (accessed on October 06, 2014). 
Todd, P. A., \& Sorkin, E. M. (1988). Diclofenac sodium. Drugs, 35(3), 244-285.

Trasande, L., Attina, T. M., \& Blustein, J. (2012). Association between urinary bisphenol A concentration and obesity prevalence in children and adolescents. Journal of the American Medical Association, 308(11), 1113-1121.

Tsai, W. T. (2006). Human health risk on environmental exposure to bisphenol-A: a review. Journal of Environmental Science and Health Part C, 24(2), 225-255.

Turiel, E., \& Martín-Esteban, A. (2010). Molecularly imprinted polymers for sample preparation: a review. Analytical Chemical Acta, 668(2), 87-99.

Ueda, M. (2010). Substances for use as bisphenol A substitutes. U.S. Patent Application $13 / 139,709$.

Umar, M., Roddick, F., Fan, L., \& Aziz, H. A. (2013). Application of ozone for the removal of bisphenol A from water and wastewater-a review. Chemosphere, 90(8), 21972207.

Umpleby, R. J., Rushton, G. T., Shah, R. N., Rampey, A. M., Bradshaw, J. C., Berch, J. K., \& Shimizu, K. D. (2001). Recognition directed site-selective chemical modification of molecularly imprinted polymers. Macromolecules, 34(24), 8446-8452.

Vallano, P. T., \& Remcho, V. T. (2000). Highly selective separations by capillary electrochromatography: molecular imprint polymer sorbents. Journal of Chromatography $A, 887(1), 125-135$. 
Vandenberg, L. N., Hauser, R., Marcus, M., Olea, N., \& Welshons, W. V. (2007). Human exposure to bisphenol A (BPA). Reproductive Toxicology, 24(2), 139-177.

Vandenberg, L. N., Maffini, M. V., Sonnenschein, C., Rubin, B. S., \& Soto, A. M. (2009). Bisphenol-A and the great divide: a review of controversies in the field of endocrine disruption. Endocrine Reviews, 30(1), 75-95.

Vasapollo, G., Sole, R. D., Mergola, L., Lazzoi, M. R., Scardino, A., Scorrano, S., \& Mele, G. (2011). Molecularly imprinted polymers: present and future prospective. International Journal of Molecular Sciences, 12(9), 5908-5945.

Welshons, W. V., Nagel, S. C., \& vom Saal, F. S. (2006). Large effects from small exposures. III. Endocrine mechanisms mediating effects of bisphenol A at levels of human exposure. Endocrinology, 147(6), s56-s69.

Whatley, H. (2001). Basic principles and modes of Capillary Electrophoresis. In Clinical and Forensic Applications of Capillary Electrophoresis. Humana Press. (pp. 21-58).

Xu, Y. (1996). Tutorial: capillary electrophoresis. The Chemical Educator, 1(2), 1-14.

Xue, X. F., WU, F., \& Deng, N. S. (2005). Determination of Endocrine Disrupting Compounds in Rivers and Lakes of Wuhan City, China. Journal of Luoyang University, 4, 010 . 
Yang, M., Ryu, J. H., Jeon, R., Kang, D., \& Yoo, K. Y. (2009). Effects of bisphenol A on breast cancer and its risk factors. Archives of Toxicology, 83(3), 281-285. 